\title{
Model Uncertainty, Recalibration, and the Emergence of Delta-Vega Hedging*
}

\author{
Sebastian Herrmann ${ }^{\dagger} \quad$ Johannes Muhle-Karbe ${ }^{\ddagger}$
}

\begin{abstract}
We study option pricing and hedging with uncertainty about a Black-Scholes reference model which is dynamically recalibrated to the market price of a liquidly traded vanilla option. For dynamic trading in the underlying asset and this vanilla option, delta-vega hedging is asymptotically optimal in the limit for small uncertainty aversion. The corresponding indifference price corrections are determined by the disparity between the vegas, gammas, vannas, and volgas of the non-traded and the liquidly traded options.
\end{abstract}

Keywords model uncertainty; recalibration; delta-vega hedging; small uncertainty aversion; asymptotics. AMS MSC 2010 Primary, 91G20, 91B16; Secondary, 93E20.

JEL Classification G13, C61, C73.

\section{Introduction}

In the context of hedging exotic derivatives, the way mathematical models for financial markets are applied in practice is often inconsistent with the assumptions these models are based on and the way they are analysed in academic research. Classical models prescribe the stochastic behaviour of certain financial variables, e.g., asset prices or interest rates, in terms of deterministic input quantities, the model's parameters. In practice, however, these deterministic parameters are often not at all treated as deterministic: exotic derivatives traders recalibrate the parameters frequently to the observed market prices of liquidly traded vanilla options and use these options to neutralise the sensitivities of their positions against changes in these parameters (appropriately called outof-model hedging by Rebonato [50]).

The benchmark Black-Scholes model is typically recalibrated by dynamic updating of the volatility parameter (that the model assumes constant) to the market price of a liquidly traded plain-vanilla option. Vega hedging1 1 then corresponds to neutralising the sensitivity of the trader's total position with respect to changes in the volatility parameter. The logical inconsistency of this practice is succinctly summarised by Rebonato [50, Section 1.3.2], for example 2

"Needless to say, out-of-model hedging is on conceptually rather shaky ground: if the volatility is deterministic and perfectly known, as many models used to arrive at the price assume it to be, there would be no need to undertake vega hedging. Furthermore, calculating the vega statistics means estimating the dependence on changes in

*The authors thank Martin Herdegen, David Hobson, Jan Kallsen, and Frank Seifried for fruitful discussions and, in particular, Martin Schweizer for pertinent remarks on the first draft. Detailed and helpful comments from two anonymous referees are also gratefully acknowledged.

${ }^{\dagger}$ University of Michigan, Department of Mathematics, 530 Church Street, Ann Arbor, MI 48109, USA, email sherrma@umich.edu Financial support by the Swiss Finance Institute is gratefully acknowledged.

$\ddagger$ University of Michigan, Department of Mathematics, 530 Church Street, Ann Arbor, MI 48109, USA, email johanmk@umich.edu

${ }^{1}$ Vega is the sensitivity of the Black-Scholes price with respect to changes in the volatility parameter.

${ }^{2}$ Davis [19] Section 2. (b)], Musiela and Rutkowski [44 Section 7.1.8], and Wilmott [58, Section 7.10.5] raise the same concern. 
volatility of a price that has been arrived at assuming the self-same volatility to be both deterministic and perfectly known. Despite these logical problems, the adoption of out-of-model hedging in general, and of vega hedging in particular, is universal in the complex-derivatives trading community."

The present paper provides a consistent justification for the use of Black-Scholes vega by acknowledging from the start that the true dynamics of the underlying are not known with certainty. We suppose that models are deemed more or less plausible depending on their "distance" from a reference Black-Scholes model for the underlying. A new feature is that the volatility parameter of the reference model is dynamically recalibrated to the observed prices of a liquidly traded vanilla option. In the limit for small aversion against this model uncertainty, delta-vega hedging then emerges naturally.

Hedging problem. Consider an agent who has sold a non-traded option on a stock $S$ with payoff $\mathrm{V}\left(S_{T}\right) \sqrt{3}$ and has access to three liquidly traded securities to hedge her exposure: the stock $S$, a vanilla option $C$ on the stock (hereafter named "call"), and a bank account with zero interest rate. In practice, the market price of the call is typically quoted in terms of its (Black-Scholes) implied volatility. That is, instead of the market price $C_{t}$, traders quote the unique $\Sigma_{t}>0$ such that

$$
C_{t}=\mathcal{C}\left(t, S_{t}, \Sigma_{t}\right)
$$

where $\mathcal{C}(t, S, \Sigma)$ is the Black-Scholes price of the call corresponding to the volatility parameter $\Sigma$. Whence, instead of modelling the dynamics of the stock and call prices, one can equivalently describe the dynamics of the stock price and the implied volatility of the call, and define the call price via (1.1).

If the stock and the call are traded using a self-financing strategy $\boldsymbol{v}=(\theta, \phi)$, the corresponding ProfitELoss (PEL) process $Y^{\boldsymbol{v}}$ has the following dynamics:

$$
\mathrm{d} Y_{t}^{\boldsymbol{v}}=\theta_{t} \mathrm{~d} S_{t}+\phi_{t} \mathrm{~d} C_{t}-\mathrm{d} \mathcal{V}\left(t, S_{t}, \Sigma_{t}\right) .
$$

Here, $\mathcal{V}\left(t, S_{t}, \Sigma_{t}\right)$ is the Black-Scholes price of the non-traded option $\mathrm{V}$ evaluated at the implied volatility $\Sigma_{t}$ backed out from the price of the call at time $t$. That is, in line with industry practice, the non-traded option is "marked to model", whereas the liquidly traded stock and call are "marked to market". However, at maturity $T$ of the non-traded option, $\mathcal{V}\left(T, S_{T}, \Sigma_{T}\right)=\mathrm{V}\left(S_{T}\right)$ is the option payoff so that $Y_{T}^{\boldsymbol{v}}$ coincides with the agent's actual terminal P\&L.

We assume that the agent is uncertain about the dynamics of the stock and the call. To wit, she considers all probability measures $P$ under which the dynamics of $(S, \Sigma)$ are governed by

$$
\begin{aligned}
& \mathrm{d} S_{t}=S_{t} \sigma_{t}^{P} \mathrm{~d} W_{t}^{0}, \\
& \mathrm{~d} \Sigma_{t}=\nu_{t}^{P} \mathrm{~d} t+\eta_{t}^{P} \mathrm{~d} W_{t}^{0}+\sqrt{\xi_{t}^{P}} \mathrm{~d} W_{t}^{1},
\end{aligned}
$$

for a Brownian motion $\left(W^{0}, W^{1}\right)$ in $\mathbb{R}^{2}$ and a process $\zeta^{P}=\left(\nu^{P}, \sigma^{P}, \eta^{P}, \xi^{P}\right)$ satisfying

$$
\nu_{t}^{P} \mathcal{C}_{\Sigma}+\frac{1}{2} S_{t}^{2} \mathcal{C}_{S S}\left(\left(\sigma_{t}^{P}\right)^{2}-\Sigma_{t}^{2}\right)+\sigma_{t}^{P} \eta_{t}^{P} S_{t} \mathcal{C}_{S \Sigma}+\frac{1}{2}\left(\left(\eta_{t}^{P}\right)^{2}+\xi_{t}^{P}\right) \mathcal{C}_{\Sigma \Sigma}=0
$$

\footnotetext{
${ }^{3}$ For simplicity, we restrict ourselves to vanilla options in this introduction. Our main result, Theorem 4.5 is also applicable to a wide range of exotic options like barrier options, lookback options, Asian options, forward-start options, and options on the realised variance of the stock.

${ }^{4} \mathrm{As}$ is customary in asymptotic analysis, the powers of the processes $\sigma^{P}, \nu^{P}, \eta^{P}$, and $\xi^{P}$ in the dynamics of $(S, \Sigma)$ are chosen so that all of them have a nontrivial effect on the leading-order term in the asymptotic expansions below. In contrast, using the uncorrelated volatility of implied volatility $\sqrt{\xi^{P}}$ instead of the uncorrelated squared volatility $\xi^{P}$ would only generate a higher-order effect. This is an artefact of the Black-Scholes model: for any reference model with a nonzero uncorrelated volatility of implied volatility, $\sqrt{\xi^{P}}$ would be the natural parametrisation; cf. Remark 3.2 for more details.

${ }^{5}$ Here, the partial derivatives $\mathcal{C}_{\Sigma}, \mathcal{C}_{S S}, \mathcal{C}_{S \Sigma}$, and $\mathcal{C}_{\Sigma \Sigma}$ of $\mathcal{C}$ are evaluated in $\left(t, S_{t}, \Sigma_{t}\right)$.
} 
The drift condition (1.3) ensures that the call price process $C$ is a local $P$-martingale 6 Note that the Black-Scholes model corresponds to $P^{0}$ with $\zeta^{P^{0}}=\zeta^{0}(\Sigma):=(0, \Sigma, 0,0)$, i.e., the implied volatility is constant and coincides with the spot volatility.

We assume that the agent has moderate risk and uncertainty aversion 7 Concerning risk aversion, we assume that in any given model, the agent seeks to maximise the expected utility from her terminal P\&L. Concerning uncertainty aversion, we suppose that she takes models less seriously the more they deviate from the reference Black-Scholes model. In the spirit of the variational preferences of Maccheroni, Marinacci, and Rustichini [42] and the multiplier preferences of Hansen and Sargent $[27,8$ this leads to the following stochastic differential game (SDG) 9

$$
v(\psi)=\sup _{\boldsymbol{v}=(\theta, \phi)} \inf _{P} E^{P}\left[U\left(Y_{T}^{\boldsymbol{v}}\right)+\frac{1}{2 \psi} \int_{0}^{T} U^{\prime}\left(Y_{t}^{\boldsymbol{v}}\right)\left|\boldsymbol{\zeta}_{t}^{P}-\boldsymbol{\zeta}_{t}^{P^{0}}\right|^{2} \mathrm{~d} t\right] .
$$

Here, $\psi>0, U$ is a utility function, the supremum runs over a suitable class of trading strategies, and the infimum is taken with respect to a suitable class of probability measures satisfying (1.2)(1.3). One interpretation is that the agent plays a game against a fictitious adversary (a "malevolent nature") who controls the true dynamics of the liquidly traded assets. However, "extreme" choices of this adversary are penalised by the positive second term in (1.4): the more the chosen model $P$ deviates from the reference Black-Scholes model $P^{0}$, the higher the penalty for the adversary. The scaling factor $\psi>0$ measures the magnitude of the agent's uncertainty aversion: small values of $\psi$ lead to high penalties even for small deviations from the Black-Scholes reference model, which means that alternative models are taken less seriously. Note that as $\boldsymbol{\zeta}_{t}^{P^{0}}=\left(0, \Sigma_{t}, 0,0\right)$, the reference Black-Scholes model reflects the belief that "the future implied volatility stays at the currently observed level." Put differently, the reference Black-Scholes model is dynamically recalibrated to the quoted option prices.

A related hedging problem without a liquidly traded call is studied in 28 for a local volatility reference model. There, the fictitious adversary chooses the true spot volatility of the stock, but is penalised according to its distance from the reference local volatility.

Asymptotics. To obtain explicit formulas, we pass to the limit where uncertainty aversion $\psi$ tends to zero 10 That is, we consider the hedging problem (1.4) as a small perturbation of the classical hedging problem in the Black-Scholes model and look for hedging strategies and price corrections that take into account the impact of model uncertainty in an asymptotically optimal manner. Our main result, Theorem 4.5. describes a hedging strategy $\boldsymbol{v}^{\star}=\left(\theta^{\star}, \phi^{\star}\right)$, a family of models $\left(P^{\psi}\right)_{\psi}$, and $\widetilde{w}_{0} \geq 0$ such that, as $\psi \downarrow 0$ :

$$
\begin{aligned}
v(\psi) & =U\left(Y_{0}\right)-U^{\prime}\left(Y_{0}\right) \widetilde{w}_{0} \psi+o(\psi) \\
& =E^{P^{\psi}}\left[U\left(Y_{T}^{\boldsymbol{v}^{\star}}\right)+\frac{1}{2 \psi} \int_{0}^{T} U^{\prime}\left(Y_{t}^{\boldsymbol{v}^{\star}}\right)\left|\boldsymbol{\zeta}_{t}^{P^{\psi}}-\boldsymbol{\zeta}_{t}^{P^{0}}\right|^{2} \mathrm{~d} t\right]+o(\psi) .
\end{aligned}
$$

The first line in (1.5) is a first-order expansion of the optimal value of the hedging problem for small values of the uncertainty aversion parameter $\psi$. The second line shows that the family

\footnotetext{
${ }^{6}$ The local martingale property of the liquidly traded assets is sufficient to exclude arbitrage opportunities. It also ensures that the agent has no incentive to invest in the market but only uses it as a hedging instrument for the non-traded option; cf. Remark 2.1]

${ }^{7}$ In contrast, most of the literature on hedging under model uncertainty studies variants of the uncertain volatility model introduced by Avellaneda, Levy, and Parás [5] and Lyons [40]. These and many more recent studies (e.g., [24, 21, 46, 49, 9, 47]) look for hedging strategies that dominate the payoff of the non-traded option almost surely for every model of a prespecified class. This worst-case approach corresponds to preferences with infinite risk and uncertainty aversion.

${ }^{8}$ We refer to [28, Section 1] for more details on these preferences and their relation to the standard expected utility framework as well as the worst-case approach.

${ }^{9}$ Our analysis also applies to somewhat more general penalty terms; cf. 2.13)-2.15). The inclusion of the term $U^{\prime}\left(Y_{t}^{v}\right)$ is not crucial but has some appealing properties. For instance, it renders the preferences invariant under affine transformations of the utility function; cf. Remark 2.6] for more details.

${ }^{10}$ Asymptotic analyses of the uncertain volatility model have been carried out by $40,2,3,23$.
} 
$\left(\boldsymbol{v}^{\star}, P^{\psi}\right)_{\psi}$ attains this optimal value at the leading order $O(\psi) 11$ More precisely, Theorem 4.5 shows that $\left(\boldsymbol{v}^{\star}, P^{\psi}\right)_{\psi}$ is in fact an asymptotic saddle point for the family of SDGs (1.4), i.e., the performance of the strategy $\boldsymbol{v}^{\star}$ is optimal at the leading order $O(\psi)$ and $\left(P^{\psi}\right)_{\psi}$ is a family of leading-order optimal choices for the fictitious adversary. The ask price at which the agent is indifferent between keeping a flat position and selling the option $\mathrm{V}$ has the expansion

$$
p_{a}(\psi)=V_{0}+\widetilde{w}_{0} \psi+o(\psi)
$$

where $V_{0}$ is the Black-Scholes price of the option $\vee$ at time 0 , evaluated with volatility $\Sigma_{0}$. Thus, $\widetilde{w}_{0} \psi$ is the leading-order premium that the agent demands as a compensation for exposing herself to model uncertainty. Accordingly, $\widetilde{w}_{0}$ measures the option's susceptibility to model misspecification and we call it the cash equivalent (of small uncertainty aversion). We next display and discuss explicit formulas for the hedging strategy $\boldsymbol{v}^{\star}$, the family of models $\left(P^{\psi}\right)_{\psi}$, and the cash equivalent $\widetilde{w}_{0}$.

The hedging strategy $\boldsymbol{v}^{\star}=\left(\theta^{\star}, \phi^{\star}\right)$ is the delta-vega hedge for the option $\mathrm{V}$ :

$$
\theta_{t}^{\star}=\mathcal{V}_{S}\left(t, S_{t}, \Sigma_{t}\right)-\phi_{t}^{\star} \mathcal{C}_{S}\left(t, S_{t}, \Sigma_{t}\right), \quad \phi_{t}^{\star}=\frac{\mathcal{V}_{\Sigma}}{\mathcal{C}_{\Sigma}}\left(t, S_{t}, \Sigma_{t}\right)
$$

To wit, the number of calls $\phi^{\star}$ is chosen so that the net vega of the agent's position, $\phi^{\star} \mathcal{C}_{\Sigma}-\mathcal{V}_{\Sigma}$, vanishes. This leaves the agent with a net $\operatorname{delt}{ }^{12}$ of $-\mathcal{V}_{S}+\phi^{\star} \mathcal{C}_{S}$ which is in turn neutralised by holding $\theta^{\star}$ shares of the underlying, so that the total portfolio is both delta- and vega-neutral 13 We emphasise that the leading-order optimality of the delta-vega hedge is independent of both the agent's utility function and her uncertainty aversion parameter $\psi$. While it is important that the agent is risk-averse (otherwise, there would be no need to hedge at all in any given model) and $i s$ moderately uncertainty-averse in our sense (vega hedging is redundant without uncertainty aversion), the precise configuration of the agent's preferences is by and large irrelevant. Moreover, note that the delta-vega hedge is computed with the currently observed implied volatility $\Sigma_{t}$ of the liquidly traded call, i.e., the Black-Scholes model used to compute the hedge is dynamically recalibrated.

We next address the asymptotically optimal models $\left(P^{\psi}\right)_{\psi}$. The process $\zeta^{P^{\psi}}$ describing the model $P^{\psi}$ satisfies

$$
\boldsymbol{\zeta}_{t}^{P^{\psi}}=\boldsymbol{\zeta}_{t}^{P^{0}}+\widetilde{\boldsymbol{\zeta}}\left(t, S_{t}, \Sigma_{t}\right) \psi+o(\psi)
$$

for some $\widetilde{\boldsymbol{\zeta}}=\widetilde{\boldsymbol{\zeta}}(t, S, \Sigma)$ arising from a linearly constrained quadratic programming problem derived from the Hamilton-Jacobi-Bellman-Isaacs (HJBI) equation associated to the SDG (1.4) (the constraints originate from the drift condition (1.3) and the restriction that the uncorrelated squared volatility of implied volatility is nonnegative). The model $P^{\psi}$ is a perturbation of the Black-Scholes model $P^{0}$, parametrised by the four processes $\nu^{P}, \sigma^{P}, \eta^{P}$, and $\xi^{P}$ in (1.2). The explicit formula for $\widetilde{\boldsymbol{\zeta}}$ (cf. (4.5)) shows that the asymptotically optimal perturbation exploits the disparity between the vegas, gammas, vannas, and volgas 14 of the non-traded option $\mathrm{V}$ and the liquidly traded call while preserving the drift condition (1.3) and the restriction $\xi^{P} \geq 0$. In fact, if each of these greeks has the same value for both the non-traded option and the liquidly traded call (e.g., if $\mathrm{V}$ is a put with the same maturity and strike as the call), then the leading-order optimal perturbation $\widetilde{\zeta}$ is zero.

Finally, we discuss the structure of the expansion (1.5) and the cash equivalent $\widetilde{w}_{0}$. As the Black-Scholes model is complete and the traded assets are local martingales, the zeroth-order term in the expansion (1.5) of $v(\psi)$ simply is the utility $U\left(Y_{0}\right)$ generated by the initial P\&L. The firstorder correction term $-U^{\prime}\left(Y_{0}\right) \widetilde{w}_{0} \psi$ is nonpositive and describes the impact of model uncertainty

\footnotetext{
${ }^{11} \mathrm{~A}$ second-order expansion and a next-to-leading order optimal strategy are obtained in [28, Theorem 3.4], where only the stock but no additional vanilla option is used for dynamic hedging.

12 Delta is the sensitivity of a Black-Scholes option value with respect to changes in the price of the underlying.

${ }^{13}$ The vega of the underlying is obviously zero.

${ }^{14}$ Gamma, vanna, and volga are the second-order partial derivatives $\partial^{2} / \partial S^{2}, \partial^{2} /(\partial S \partial \Sigma)$, and $\partial^{2} / \partial \Sigma^{2}$ of the Black-Scholes value of an option.
} 
for small uncertainty aversion. The cash equivalent $\widetilde{w}_{0}$ is determined by a linear second-order parabolic partial differential equation (PDE) with a source term. It has the following probabilistic representation:

$$
\begin{aligned}
& \widetilde{w}_{0}=\frac{1}{2} E^{P^{0}}\left[\int_{0}^{T} \widetilde{g}\left(t, S_{t}, \Sigma_{0}\right) \mathrm{d} t\right], \quad \text { with } \\
& \widetilde{g}(t, S, \Sigma)=-\Sigma(\underbrace{\phi^{\star} S^{2} \mathcal{C}_{S S}-S^{2} \mathcal{V}_{S S}}_{\text {net cash gamma }}) \widetilde{\sigma}-\Sigma(\underbrace{\phi^{\star} S \mathcal{C}_{S \Sigma}-S \mathcal{V}_{S \Sigma}}_{\text {net cash vanna }}) \widetilde{\eta}-\frac{1}{2}(\underbrace{\phi^{\star} \mathcal{C}_{\Sigma \Sigma}-\mathcal{V}_{\Sigma \Sigma}}_{\text {net volga }}) \widetilde{\xi} \geq 0 .
\end{aligned}
$$

Here, $\widetilde{\boldsymbol{\zeta}}(t, S, \Sigma)=(\widetilde{\nu}, \widetilde{\sigma}, \widetilde{\eta}, \widetilde{\xi})(t, S, \Sigma)$ and all functions on the right-hand side of (1.6) are evaluated in $(t, S, \Sigma)$. The cash equivalent $\widetilde{w}_{0}$ is thus determined by the expected net cash gamma, net cash vanna, and net volga of the delta-vega hedged position that is accumulated over the lifetime of the option $\mathrm{V} 15$ These three net (cash) greeks are weighted by the leading-order optimal perturbation of the spot volatility, the correlated volatility of implied volatility, and the uncorrelated squared volatility of implied volatility, respectively. The larger $\widetilde{g}$, the larger the cash equivalent $\widetilde{w}_{0}$. In particular, a short net gamma position (after vega hedging) is exposed to high spot volatility (positive $\tilde{\sigma}$ ), a short net vanna position is exposed to volatility of implied volatility that is positively correlated with the underlying (positive $\widetilde{\eta}$ ), and a short net volga position is exposed to volatility of implied volatility (positive $\widetilde{\xi})[16$ Conversely, long positions in net gamma or net vanna have the reverse exposures, but a long net volga position is not exposed to volatility of implied volatility because $\widetilde{\xi}$ cannot be negative.

Techniques. The HJBI equation associated to the SDG (1.4) involves a pointwise min-max problem for the hedging strategy of the agent and for the control variables of the fictitious adversary. This min-max problem has a nonlinear equality constraint and an inequality constraint that originate from the drift condition (1.3) and the restriction $\xi^{P} \geq 0$, respectively.

Formally passing to the limit as $\psi \downarrow 0$, this problem can be approximated by a linearly constrained quadratic minimisation problem and an unconstrained quadratic maximisation problem. Both of these problems can be solved explicitly and give rise to the delta-vega hedge and candidate controls $\left(\boldsymbol{\zeta}^{\psi}\right)_{\psi}$ corresponding approximately to the family of models $\left(P^{\psi}\right)_{\psi}$. Plugging these candidates back into the HJBI equation yields a PDE for the first-order term in the expansion of the value function of the SDG.

The rigorous verification of the (asymptotic) optimality of these candidates combines an asymptotic analysis of the HJBI equation with classical verification arguments for SDGs. It is divided into a purely analytic and a probabilistic part. Due to the constraints in the min-max problem, both parts of the proof require substantially different approaches compared to those used in [28]. The analytic part uses careful direct estimates and Lagrange duality theory for constrained optimisation problems to show that the candidate value function is asymptotically (in a suitable sense) a solution to the HJBI equation. The probabilistic part of the proof adapts classical verification arguments for SDGs to the asymptotic setting. New difficulties arise now from the fact that the candidate controls of the fictitious adversary do not satisfy the drift condition (1.3) exactly (as the nonlinear constraint is only approximated by a linear one).

Related literature. Let us now put our results in context by discussing some of the extant literature on the hedging of exotics using vanilla options. One strand of literature postulates that both the asset price and its spot volatility are stochastic and follow given dynamics driven by two Brownian motions. Stochastic volatility models of this type can typically be completed by using a single plain-vanilla option as an extra hedging instrument in addition to the underlying

\footnotetext{
${ }^{15}$ In contrast, if there is no liquidly traded call available as a hedging instrument, then the option's cash gamma is the only greek that appears in the probabilistic representation of the cash equivalent [28].

${ }^{16}$ According to formula (1.6), a short net volga position is only exposed to the part of the volatility of implied volatility that is uncorrelated with the underlying. However, it can be seen from the proof that the correlated volatility of implied volatility has the same effect, albeit only at the order $O\left(\psi^{2}\right)$.
} 
stock 17 In Markovian settings, replicating strategies can then be determined in close analogy to the classical Black-Scholes argument. This leads to the so-called "delta-sigma hedge" [34, 56], which neutralises the portfolio's sensitivity with respect to changes in both the underlying stock price and the spot volatility. This strategy is related to the delta-vega hedge in that it also makes use of the derivative of the option price with respect to "volatility". Here, however, "volatility" refers to the spot volatility that can (at least in theory) be backed out from the realised variance of the stock. Instead, delta-vega hedging neutralises a portfolio's sensitivity with respect to changes in the (Black-Scholes) implied volatility that is deduced from the market price of a liquidly traded option. While the spot volatility gives the instantaneous volatility of the stock price, the implied volatility is rather an estimate for the future volatility realised over the whole time interval ranging from today to the maturity of the liquidly traded option. Moreover, in practice, also stochastic volatility models have to be recalibrated once the model and market prices of liquidly traded options diverge.

Another strand of literature studies the robust hedging of exotic derivatives. To wit, these studies look for hedging strategies that work in some large class of models (e.g., any continuous martingale model). The hedging strategies are typically of semi-static form: they allow a static position in a portfolio of calls and puts (often for one maturity and all strikes) and dynamic trading in the underlying 18 For variance swaps, this leads to a robust replicating strategy [45, whereas robust sub- and superhedging strategies have been determined for various other exotic options (cf., e.g., 29, 12, 18, 17, 16, 32, 30, 31]). In these studies, the goal is to find portfolios that sub- or superreplicate the exotic option in each possible scenario 19 The underlying preferences therefore correspond to infinite aversion both against risk in a given model and uncertainty about the model itself. In contrast, as in [28], we consider a more moderate attitude towards risk and uncertainty that interpolates smoothly between the worst-case approach and the classical setting with one fixed model. The other major difference is that we allow dynamic trading in a single vanilla option instead of static positions in puts and calls of many strikes.

In practice, even the most liquid at-the-money options have substantially larger bid-ask spreads than the underlying stocks. As a result, a direct implementation of the delta-vega hedge with, e.g., daily rebalancing leads to substantial transaction costs and is found to be inferior to semistatic alternatives in several case studies [17, 48]. As a remedy, the delta-vega hedge needs to be implemented with a suitable "buffer". That is, rebalancing trades should only take place once the hedge portfolio deviates sufficiently from its frictionless target. The corresponding trading boundaries for Black-Scholes delta-hedging strategies have been determined explicitly in the smallcost limit by [57]; cf. also [37] and the references therein for extensions to more general settings. Extending these tracking results to more general target strategies involving liquid vanilla options is a major challenge for future research. To date, the only result of this kind concerns the dynamic trading of options to reduce transaction costs [26], which leads to a buffered version of the deltagamma hedge.

Organisation of the paper. The remainder of the article is organised as follows. The mathematical framework for the hedging problem under model uncertainty is introduced in Section 2 . Section 3 outlines the heuristic derivation of the asymptotically optimal solution. Our main results are stated and discussed in Section 4 Finally, all proofs are relegated to Section 5

Notation. Vectors $\mathbf{a} \in \mathbb{R}^{n}$ and vector-valued functions are printed in boldface type. The transpose of a vector $\mathbf{a}$ is denoted by $\mathbf{a}^{\top}$ and its Euclidean norm by $|\mathbf{a}|$. For the sake of readability, we mostly suppress the arguments of functions in the notation. In calculations and estimates, we typically display the arguments only on the left-most side of (in-)equalities; the omitted arguments should then be clear from the context. Partial derivatives of functions with respect to

\footnotetext{
${ }^{17}$ See, e.g., [52 19, 20] for precise conditions.

${ }^{18}$ Semi-static hedging problems have also been analysed numerically in the context of the Lagrangian uncertain volatility model [6] 4].

${ }^{19}$ General superhedging duality results in the semi-static context have been obtained, among others, by [1, 7] 22, 25. 10; see also the references therein.
} 
scalar variables are denoted by subscripts as in (1.3) and $\mathrm{D}_{\zeta} H$ denotes the gradient of a function $H(\ldots ; \boldsymbol{\zeta})$ with respect to the vector variable $\boldsymbol{\zeta}$.

\section{Problem formulation}

To allow for dynamic trading in both the stock and an option on the stock, we consider market models for the joint evolution of both assets. Instead of prescribing the dynamics of the option, we follow Schönbucher's approach [53] and model its Black-Scholes implied volatility20 This approach is outlined in Section 2.1 and motivates the precise setup introduced in Section 2.2. The hedging problem is in turn formulated in Section 2.3

\subsection{Market models for the underlying and its implied volatility}

We consider a financial market with three liquidly traded securities: a stock $S$, an option written on the stock, and a bank account with zero interest rate. The liquidly traded option has a payoff of the form $\mathrm{C}\left(S_{T_{\mathrm{C}}}\right)$ at maturity $T_{\mathrm{C}}$. To avoid confusion with the non-traded option introduced later, this liquidly traded option will be named "call" hereafter. It is market practice to quote option prices in terms of their (Black-Scholes) implied volatilities. That is, traders do not quote the market price $p$ of the call $C$, but instead the unique $\Sigma>0$ such that $p=\mathcal{C}\left(t, S_{t}, \Sigma\right)$, where $\mathcal{C}(\cdot, \cdot, \Sigma)$ is the solution of the Black-Scholes PDE

$$
\begin{aligned}
\mathcal{C}_{t}(t, S, \Sigma)+\frac{1}{2} \Sigma^{2} S^{2} \mathcal{C}_{S S}(t, S, \Sigma) & =0, \quad(t, S) \in\left(0, T_{\mathrm{C}}\right) \times \mathbb{R}_{+}, \\
\mathcal{C}\left(T_{\mathrm{C}}, S, \Sigma\right) & =\mathrm{C}(S), \quad S \in \mathbb{R}_{+},
\end{aligned}
$$

corresponding to volatility $\Sigma$, maturity $T_{\mathrm{C}}$, and the terminal payoff $\mathrm{C}\left(S_{T_{\mathrm{C}}}\right)$ of the call. Following this practice and Schönbucher's approach [53, we model the implied volatility rather than the price process of the call. To wit, we assume that the joint dynamics of the stock $S$ and the call's implied volatility $\Sigma$ are governed by

$$
\begin{aligned}
\mathrm{d} S_{t} & =S_{t} \sigma_{t} \mathrm{~d} W_{t}^{0}, \\
\mathrm{~d} \Sigma_{t} & =\nu_{t} \mathrm{~d} t+\eta_{t} \mathrm{~d} W_{t}^{0}+\sqrt{\xi_{t}} \mathrm{~d} W_{t}^{1},
\end{aligned}
$$

for a bivariate standard Brownian motion $\left(W^{0}, W^{1}\right)$ and processes $\sigma, \nu, \eta, \xi$. Here, $\sigma$ is the spot volatility, and $\nu, \eta$, and $\xi$ correspond to the drift of implied volatility, the correlated volatility of implied volatility, and the uncorrelated square 212 volatility of implied volatility, respectively. The price process $C$ of the call in turn is

$$
C_{t}=\mathcal{C}\left(t, S_{t}, \Sigma_{t}\right)
$$

By Itô's formula, its dynamics are given by

$$
\begin{array}{rl}
\mathrm{d} C_{t}=\mathrm{d} \mathcal{C}( & \left.t, S_{t}, \Sigma_{t}\right)=\mathcal{C}_{t} \mathrm{~d} t+\mathcal{C}_{S} \mathrm{~d} S_{t}+\mathcal{C}_{\Sigma} \mathrm{d} \Sigma_{t}+\frac{1}{2} \mathcal{C}_{S S} \mathrm{~d}\langle S\rangle_{t}+\mathcal{C}_{S \Sigma} \mathrm{d}\langle S, \Sigma\rangle_{t}+\frac{1}{2} \mathcal{C}_{\Sigma \Sigma} \mathrm{d}\langle\Sigma\rangle_{t} \\
=\mathcal{C}_{S} & \mathrm{~d} S_{t}+\eta_{t} \mathcal{C}_{\Sigma} \mathrm{d} W_{t}^{0}+\sqrt{\xi_{t}} \mathcal{C}_{\Sigma} \mathrm{d} W_{t}^{1} \\
& +\left\{\mathcal{C}_{t}+\nu_{t} \mathcal{C}_{\Sigma}+\frac{1}{2} \sigma_{t}^{2} S_{t}^{2} \mathcal{C}_{S S}+\sigma_{t} \eta_{t} S_{t} \mathcal{C}_{S \Sigma}+\frac{1}{2}\left(\eta_{t}^{2}+\xi_{t}\right) \mathcal{C}_{\Sigma \Sigma}\right\} \mathrm{d} t .
\end{array}
$$

We suppose that all liquidly traded assets are local martingales (cf. Footnote 6 and Remark 2.1). Thus, the drift of the liquidly traded call must vanish. Using the PDE (2.1) to substitute $\mathcal{C}_{t}=$

\footnotetext{
${ }^{20}$ Other early articles on risk-neutral dynamics for stochastic implied volatility models include [41, 11 39. For more recent developments on arbitrage-free market models for (parts of or the whole) option price surface, we refer the reader to [55, 54, 13, 35, 14, 15, 36] and the references therein.

${ }^{21}$ The parametrisation in terms of the squared volatility of implied volatility is explained in Remark 3.2.
} 
$\mathcal{C}_{t}\left(t, S_{t}, \Sigma_{t}\right)$, the following drift condition obtains (cf. [53, Equation (3.6)]):

$$
\nu_{t} \mathcal{C}_{\Sigma}+\frac{1}{2} S_{t}^{2} \mathcal{C}_{S S}\left(\sigma_{t}^{2}-\Sigma_{t}^{2}\right)+\sigma_{t} \eta_{t} S_{t} \mathcal{C}_{S \Sigma}+\frac{1}{2}\left(\eta_{t}^{2}+\xi_{t}\right) \mathcal{C}_{\Sigma \Sigma}=0
$$

In view of (2.5), at most three of the four processes $\nu, \sigma, \eta$, and $\xi$ can be chosen arbitrarily for the resulting model to satisfy the drift condition. Further natural restrictions are $\sigma>0, \xi \geq 0$, and $\Sigma>0$. Note that the standard Black-Scholes model corresponds to the choice $\nu=\eta=\xi=0$ and $\sigma_{t}=\Sigma_{t}=\Sigma_{0}$. Then, the drift condition (2.5) is clearly satisfied and spot and implied volatilities are constant and identical.

Remark 2.1. Let us briefly discuss as in [28, Remark 2.2] why we assume that the traded assets $S$ and $C$ have zero drifts. With nonzero drifts, the agent would use the traded assets not only as hedging instruments, but also as investment vehicles. This would complicate the analysis considerably as the limiting P\&L process would no longer be constant but stochastic. But the real-world drift rates usually have little impact on the hedging component, i.e., the difference between a utility-based hedging strategy and the corresponding utility-based optimal investment strategy 22 Assuming that the traded assets have zero drifts allows us to focus on hedging rather than optimal investment. Indeed, the agent then has no incentive to trade the stock and the call other than as hedging instruments for the non-traded option.

In the following Section 2.2, we introduce a setup to formulate our hedging problem with uncertainty about the processes $\nu, \sigma, \eta, \xi$.

\subsection{Model uncertainty setup}

Fix a time horizon $T>0$ and constants $S_{0}>0, \Sigma_{0}>0$, and $A_{0} \in \mathbb{R}$. Let

$$
\Omega=\left\{\omega=\left(\omega_{t}^{S}, \omega_{t}^{\Sigma}, \omega_{t}^{A}\right)_{t \in[0, T]} \in C\left([0, T] ; \mathbb{R}^{3}\right): \omega_{0}=\left(S_{0}, \Sigma_{0}, A_{0}\right)\right\}
$$

be the canonical space of continuous paths in $\mathbb{R}^{3}$ starting in $\left(S_{0}, \Sigma_{0}, A_{0}\right)$, endowed with the topology of uniform convergence. Moreover, let $\mathcal{F}$ be the Borel $\sigma$-algebra on $\Omega$. We denote by $\left(S_{t}\right)_{t \in[0, T]}$, $\left(\Sigma_{t}\right)_{t \in[0, T]}$, and $\left(A_{t}\right)_{t \in[0, T]}$ the first, second, and third component of the canonical process, respectively, i.e., $S_{t}(\omega)=\omega_{t}^{S}, \Sigma_{t}(\omega)=\omega_{t}^{\Sigma}$, and $A_{t}(\omega)=\omega_{t}^{A}$. We write $\mathbb{F}=\left(\mathcal{F}_{t}\right)_{t \in[0, T]}$ for the (raw) filtration generated by $(S, \Sigma, A)$, and denote by $M_{t}:=\sup _{u \in[0, t]} S_{u}, t \in[0, T]$, the running maximum of $S$. Unless otherwise stated, all probabilistic notions requiring a filtration, such as progressive measurability etc., pertain to $\mathbb{F}$. Finally, we write $\left(\mathbf{X}_{t}\right)_{t \in[0, T]}$ for the vector-valued process $\mathbf{X}_{t}=\left(S_{t}, A_{t}, M_{t}, \Sigma_{t}\right)$.

Remark 2.2. The processes $S, M$, and $\Sigma$, model the stock price, its running maximum, and the implied volatility of the traded call, respectively. The process $A$ is an additional state variable that can be used to track exotic features of the non-traded option the agent has to hedge. For instance, an Asian call option with strike $K>0$ has the payoff $\left(\frac{1}{T} \int_{0}^{T} S_{t} \mathrm{~d} t-K\right)^{+}$. Setting $A_{t}=\int_{0}^{t} S_{u} \mathrm{~d} u$, the payoff can be recast as $\left(\frac{1}{T} A_{T}-K\right)^{+}$and exploiting the Markovian structure of the process $(S, A)$, the Black-Scholes value of the Asian call can be written as a function $\mathcal{V}\left(t, S_{t}, A_{t}\right)$ of time, the current stock price, and the current value of the additional state variable $A$.

We now introduce a large class of probability measures on $(\Omega, \mathcal{F})$ that will serve as alternative models for the evolution of the traded assets.

Definition 2.3. $\mathfrak{P}^{00}$ is the set of probability measures on $(\Omega, \mathcal{F})$ for which there exists a quadruple $\zeta^{P}=\left(\nu_{t}^{P}, \sigma_{t}^{P}, \eta_{t}^{P}, \xi_{t}^{P}\right)_{t \in[0, T]}$ of real-valued progressively measurable processes such that:

\footnotetext{
${ }^{22}$ For example, [33] find in a Lévy model that the (drift-dependent) variance-optimal hedge is virtually identical to the (drift-independent) Black-Scholes delta hedge.
} 
(a) $S$ and $\Sigma-\int_{0}^{\cdot} \nu_{t}^{P} \mathrm{~d} t$ are (continuous) local $P$-martingales with quadratic (co-)variations

$$
\begin{aligned}
\mathrm{d}\langle S\rangle_{t} & =S_{t}^{2}\left(\sigma_{t}^{P}\right)^{2} \mathrm{~d} t, \\
\mathrm{~d}\langle\Sigma\rangle_{t} & =\left(\left(\eta_{t}^{P}\right)^{2}+\xi_{t}^{P}\right) \mathrm{d} t, \\
\mathrm{~d}\langle S, \Sigma\rangle_{t} & =S_{t} \sigma_{t}^{P} \eta_{t}^{P} \mathrm{~d} t ;
\end{aligned}
$$

(b) $S$ and $\Sigma$ are $P$-a.s. positive;

(c) $\xi^{P} \geq 0$ P-a.s.;

(d) the drift condition

$$
\nu_{t}^{P} \mathcal{C}_{\Sigma}+\frac{1}{2} S_{t}^{2} \mathcal{C}_{S S}\left(\left(\sigma_{t}^{P}\right)^{2}-\Sigma_{t}^{2}\right)+\sigma_{t}^{P} \eta_{t}^{P} S_{t} \mathcal{C}_{S \Sigma}+\frac{1}{2}\left(\left(\eta_{t}^{P}\right)^{2}+\xi_{t}^{P}\right) \mathcal{C}_{\Sigma \Sigma}=0
$$

holds $\mathrm{d} t \times P$-a.e. Here, the partial derivatives of $\mathcal{C}$ are evaluated in $\left(t, S_{t}, \Sigma_{t}\right)$.

A probability measure $P \in \mathfrak{P}^{00}$ is called a model and the process $\zeta^{P}$ is referred to as the control corresponding to the model $P$. Each $P$ represents a market model for the stock price $S$ and the implied volatility $\Sigma$ with dynamics of the form (2.2)-(2.3) (with $\sigma$ replaced by $\sigma^{P}$ etc.) and (2.7) guarantees that the call price process is a local $P$-martingale (cf. (2.5)).

Definition 2.4. The function $\zeta^{0}: \mathbb{R}_{+} \rightarrow \mathbb{R}^{4}$ given by $\zeta^{0}(\Sigma)=(0, \Sigma, 0,0)^{\top}$ is called reference feedback control. A probability measure $P \in \mathfrak{P}^{00}$ such that $\boldsymbol{\zeta}_{t}^{P}=\zeta^{0}\left(\Sigma_{t}\right) \mathrm{d} t \times P$-a.e. is called reference model.

Note that a reference model corresponds to a Black-Scholes model with constant volatility $\sigma_{t} \equiv \Sigma_{t} \equiv \Sigma_{0}$ and trivially satisfies the drift condition (2.7).

Next, we consider a subclass $\mathfrak{P}^{0} \subset \mathfrak{P}^{00}$ which (in contrast to $\mathfrak{P}^{00}$ ) also prescribes the dynamics for the additional state variable $A$ that tracks exotic features of the non-traded option. To this end, we fix Borel functions $\alpha, \beta, \gamma, \delta:[0, T] \times \mathbb{R}^{3} \rightarrow \mathbb{R}$.

Definition 2.5. $\mathfrak{P}^{0}=\mathfrak{P}^{0}(\alpha, \beta, \gamma, \delta) \subset \mathfrak{P}^{00}$ is the subset of probability measures $P$ such that $A$ is a (continuous) $P$-semimartingale with canonical decomposition

$$
\mathrm{d} A_{t}=\left(\alpha+\frac{\left(\sigma_{t}^{P}\right)^{2}}{2} \beta\right) \mathrm{d} t+\gamma \mathrm{d} S_{t}+\delta \mathrm{d} M_{t}
$$

under $P$ (the functions $\alpha, \beta, \gamma, \delta$ are evaluated in $\left(t, S_{t}, A_{t}, M_{t}\right)$ ).

The form (2.8) for the dynamics of $A$ is flexible enough to express Black-Scholes values of, e.g., Asian options, options on the realised variance, or forward-start options by PDE methods. We also note that given sufficiently regular functions $\alpha, \beta, \gamma, \delta$, there is a unique reference model in $\mathfrak{P}^{0}$.

\subsection{Hedging problem}

Dynamic model recalibration. Consider an agent who has sold a non-traded option (possibly exotic) on $S$ with sufficiently regular 23 payoff $\vee\left(S_{T}, A_{T}, M_{T}\right)^{24}$ at maturity $T$. She can hedge her exposure by trading dynamically and frictionlessly in the stock, the call, and the bank account.

Among all possible dynamics, the agent considers as most plausible the Black-Scholes model corresponding to the currently observed implied volatility, i.e., $\nu=\eta=\xi=0$ and $\sigma=\Sigma$ (recall that the drift condition (2.5) holds for this choice). This corresponds to the reference belief that

\footnotetext{
${ }^{23}$ See Assumption 4.2 for the precise details.

${ }^{24}$ Recall that $M$ is the running maximum of $S$ and that $A$ is a general state variable with dynamics of the form (2.8) which can track exotic features of the option like the average stock price or the stock price at an intermediate time; cf. [28, Section 4.2] for examples.
} 
"The future implied volatility stays at the currently observed level." Note that this differs from the conviction that "The future implied volatility equals the implied volatility observed at time 0.": the former belief allows for dynamic updating of the observed implied volatility, the latter does not. In particular, at each time $t$, the agent (re-)calibrates her Black-Scholes model to the observed market price of the liquidly traded call option. This is in line with the market practice of frequent recalibration of pricing models to observed option prices. The corresponding BlackScholes value of the non-traded option can readily be obtained by PDE methods. To this end, let $\mathbf{G}=\mathbb{R}_{+} \times \mathbb{R} \times \mathbb{R}_{+}$be the state space of the process $(S, A, M)$ and for each $\Sigma>0$, let $\mathcal{V}(\cdot, \Sigma)$ be a classical solution to the PDE

$$
\begin{aligned}
\mathcal{V}_{t}+\left(\alpha+\frac{1}{2} \beta \Sigma^{2}\right) \mathcal{V}_{A}+\frac{1}{2} \Sigma^{2} S^{2}\left(\mathcal{V}_{S S}+2 \gamma \mathcal{V}_{S A}+\gamma^{2} \mathcal{V}_{A A}\right) & =0 & & \text { on }(0, T) \times \mathbf{G}, \\
\delta \mathcal{V}_{A}+\mathcal{V}_{M} & =0 & & \text { on }\{(t, S, A, M): S \geq M\} \\
\mathcal{V}(T, \cdot, \Sigma) & =\mathrm{V} & & \text { on } \mathbf{G} .
\end{aligned}
$$

Define the process $V=\left(V_{t}\right)_{t \in[0, T]}$ by

$$
V_{t}=\mathcal{V}\left(t, S_{t}, A_{t}, M_{t}, \Sigma_{t}\right)
$$

Then, as is well known, $V_{t}$ is the Black-Scholes value at time $t$ of the non-traded option $\mathrm{V}$ given the current observation of the stock price $S_{t}$, the state variables $A_{t}$ and $M_{t}$, and the implied volatility $\Sigma_{t}$. In other words, the Black-Scholes model used to value the option $\mathrm{V}$ is dynamically recalibrated to the observed call prices.

Trading strategies and Profit\&Loss processes. A (self-financing) trading strategy is represented by a pair $\boldsymbol{v}=(\theta, \phi)$ of real-valued, locally bounded 25 progressively measurable processes $\theta=\left(\theta_{t}\right)_{t \in[0, T]}$ and $\phi=\left(\phi_{t}\right)_{t \in[0, T]}$, which describe the number of stocks and calls held by the agent, respectively. Fix a constant $Y_{0} \in \mathbb{R}$ and for each $P \in \mathfrak{P}^{0}$ and any trading strategy $\boldsymbol{v}$, define the Profit\&Loss (PELL) process $Y^{\boldsymbol{v}, P}=\left(Y_{t}^{\boldsymbol{v}, P}\right)_{t \in[0, T]}$ by

$$
Y_{t}^{\boldsymbol{v}, P}=Y_{0}+V_{0}+\int_{0}^{t} \theta_{u} \mathrm{~d} S_{u}+\int_{0}^{t} \phi_{u} \mathrm{~d} C_{u}-V_{t} .
$$

Here, the stochastic integrals are constructed under $P$. The process $Y_{t}^{\boldsymbol{v}, P}$ describes the value of the agent's portfolio at time $t$ under the model $P$, i.e., her initial capital $Y_{0}+V_{0}$ (recall the definition of $V_{t}$ in (2.10) ) plus gains from self-financing trading in the liquidly traded assets (computed under $P$ ) minus the (recalibrated) Black-Scholes value $V_{t}$ of the non-traded option at time $t$. Note that while the position in the liquidly traded assets are "marked to market" and constitute "real values" (because these assets could be liquidated instantly by assumption), the non-traded option has to be "marked to model" and thus only has a "theoretical value". However, at the maturity $T$ of the non-traded option, $V_{T}$ equals the option's payoff and the value of the option becomes "real". In particular, $Y_{T}^{\boldsymbol{v}, P}$ is the agent's actual terminal wealth.

Uncertainty aversion. Fix a model set $\mathfrak{P} \subset \mathfrak{P}^{0}$ and a set $\mathfrak{Y}$ of trading strategies. Similarly to 28,26 we assume that the agent ranks trading strategies in $\mathfrak{Y}$ according to a numerical representation of her preferences of the form

$$
\inf _{P \in \mathfrak{P}} E^{P}\left[U\left(Y_{T}^{\boldsymbol{v}, P}\right)+\frac{1}{\psi} \int_{0}^{T} U^{\prime}\left(Y_{t}^{\boldsymbol{v}, P}\right) f\left(\Sigma_{t}, \boldsymbol{\zeta}_{t}^{P}\right) \mathrm{d} t\right],
$$

\footnotetext{
${ }^{25}$ For locally bounded, progressively measurable integrands, the stochastic integrals in (2.11) are well defined under each measure in $\mathfrak{P}^{0}$. The delta-vega hedge considered in our main result, Theorem 4.5 is even continuous.

${ }^{26}$ In [28, only the underlying but no liquid call is available for dynamic hedging and the spot volatility is the only control variable of the fictitious adversary.
} 
where $f$ is a suitable function such that for each $\Sigma>0, \mathbb{R}^{4} \ni \boldsymbol{\zeta} \mapsto f(\Sigma, \boldsymbol{\zeta})$ is strictly convex with a unique minimum of 0 at the reference point $\zeta^{0}(\Sigma)$. The utility function $U$ describes the agent's attitude towards risk in a given model. The infimum over models in $\mathfrak{P}$ together with the penalty term 27 (the second summand inside the expectation in (2.12) ) expresses her attitude towards model uncertainty. The parameter $\psi>0$ quantifies the magnitude of her uncertainty aversion. Indeed, in the limit $\psi \downarrow 0$, the second summand in (2.12) converges to the indicator $+\infty \mathbf{1}_{\left\{\boldsymbol{\zeta}^{P} \neq \boldsymbol{\zeta}^{0}(\Sigma)\right\}}$ and the criterion (2.12) collapses to the standard expected utility under the reference model. In this case, the agent faces no uncertainty aversion at all as she only deems the reference model plausible. Conversely, in the limit $\psi \uparrow \infty$, the penalty term converges to 0 for all $P \in \mathfrak{P}$ and the criterion (2.12) becomes the familiar worst-case expectation $\inf _{P \in \mathfrak{P}} E^{P}\left[U\left(Y_{T}^{\boldsymbol{v}, P}\right)\right]$. In this case, the agent is very uncertainty-averse in that she regards every model in $\mathfrak{P}$ as equally plausible. The criterion (2.12) interpolates smoothly between these two extreme cases. The reference model is not penalised, while alternative models are underweighted in the agent's decision making according to their "distance" from the reference model. The interpretation is that the reference model is considered most plausible. Alternative models are taken less seriously, but not ruled out a priori.

For tractability, we focus on the following quadratic specification for the penalty function $f 28$

$$
\begin{aligned}
f(\Sigma, \boldsymbol{\zeta}) & =\frac{1}{2}\left(\boldsymbol{\zeta}-\boldsymbol{\zeta}^{0}(\Sigma)\right)^{\top} \Psi^{-1}\left(\boldsymbol{\zeta}-\boldsymbol{\zeta}^{0}(\Sigma)\right) \\
& =\frac{1}{2}\left(\nu^{2} / \psi_{\nu}+(\sigma-\Sigma)^{2} / \psi_{\sigma}+\eta^{2} / \psi_{\eta}+\xi^{2} / \psi_{\xi}\right)
\end{aligned}
$$

where

$$
\Psi=\operatorname{diag}\left(\psi_{\nu}, \psi_{\sigma}, \psi_{\eta}, \psi_{\xi}\right) \text { and } \psi_{\nu}, \psi_{\sigma}, \psi_{\eta}, \psi_{\xi}>0
$$

The parameters $\psi_{\nu}, \psi_{\sigma}, \psi_{\eta}, \psi_{\xi}$ describe the agent's relative uncertainty about the true drift of implied volatility, spot volatility, correlated volatility of implied volatility, and uncorrelated squared volatility of implied volatility, respectively. The scaling parameter $\psi$ measures her overall level of aversion against uncertainty.

Remark 2.6. Let us argue as in [28, Remark 2.6] why we include the term $U^{\prime}\left(Y_{t}^{\boldsymbol{v}, P}\right)$ in the penalty term of the numerical representation (2.12) 29 First, in the standard expected utility framework, preferences are invariant under affine transformations of the utility function. The term $U^{\prime}\left(Y_{t}^{\boldsymbol{v}, P}\right)$ ensures that this property is preserved for uncertainty-averse decision makers whose preferences are described by (2.12). Second, $U^{\prime}\left(Y_{t}^{\boldsymbol{v}, P}\right)$ (rather than, e.g., $U^{\prime}\left(Y_{0}\right)^{30}$ ) is the natural choice for a dynamic formulation of the hedging problem (2.16) in terms of a family of conditional problems parametrised by the initial time $t$, stock price $S_{t}=s$, and P\&L $Y_{t}^{\boldsymbol{v}, P}=y$. Third, our results show that the preferences described by (2.12) have approximately "constant uncertainty aversion" in the sense that the cash equivalent $\widetilde{w}_{0}$ does not depend on the P\&L (cf. Proposition 4.6). This would not be the case if one omitted the term $U^{\prime}\left(Y_{t}^{\boldsymbol{v}, P}\right)$ in (2.12) 31

\footnotetext{
${ }^{27}$ Note that the penalty is imposed on the fictitious adversary who chooses the model $P$ after the agent has chosen her trading strategy $\boldsymbol{v}$. Alternatively, it can be interpreted as a fictitious bonus for the agent.

${ }^{28}$ More general functions $f$ are considered in 28 , where it becomes apparent that only the locally quadratic structure at the minimum matters for the leading-order asymptotics.

${ }^{29}$ Formally, this corresponds to directly imposing the penalty in monetary terms, i.e., inside the utility function in 2.12.

${ }^{30} \mathrm{U} \operatorname{sing} U^{\prime}\left(Y_{0}\right)$ instead of $U^{\prime}\left(Y_{t}^{\boldsymbol{v}, P}\right)$ would yield the same expansion for $v(\psi)$ as in Theorem 4.5 Formally, the delta-vega hedge and the candidate optimal controls for the fictitious adversary would still be leading-order optimal. This is because the P\&L process converges to a constant in the limit of small uncertainty aversion. Consequently, one could also remove $U^{\prime}\left(Y_{t}^{\boldsymbol{v}, P}\right)$ from the penalty term by replacing the matrix $\Psi$ by $\Psi / U^{\prime}\left(Y_{0}\right)$. Then $U^{\prime}\left(Y_{0}\right)$ would reappear in the candidate feedback control for the fictitious adversary and hence also in the cash equivalent $\widetilde{w}_{0}$. Keeping $U^{\prime}\left(Y_{t}^{\boldsymbol{v}}, P\right)$ in the penalty term avoids that the candidate optimal controls depend on the current P\&L of the agent. This avoids some mathematical subtleties in the formulation of the hedging problem; cf. 28, where the P\&L process $Y$ lives on the canonical space so that (progressively measurable) controls may depend on $Y$.

${ }^{31}$ In the context of robust portfolio choice, Maenhout [3] also observes that some modification of the standard (non wealth-dependent) entropic penalty is reasonable to avoid that the agent's uncertainty aversion wears off as her wealth rises, and tackles this effect by directly modifying the HJBI equation.
} 
We also note that the penalty term depends on the chosen trading strategy $\boldsymbol{v}$ of the agent only through her current P\&L (just as the indirect risk tolerance process of an investor depends on her trading strategy only through her current wealth).

Hedging problem. Fix $\psi>0$. For each trading strategy $\boldsymbol{v} \in \mathfrak{Y}$ and each model $P \in \mathfrak{P}$, we define the objective of our hedging problem by

$$
J^{\psi}(\boldsymbol{v}, P):=E^{P}\left[U\left(Y_{T}^{\boldsymbol{v}, P}\right)+\frac{1}{\psi} \int_{0}^{T} U^{\prime}\left(Y_{t}^{\boldsymbol{v}, P}\right) f\left(\Sigma_{t}, \boldsymbol{\zeta}_{t}^{P}\right) \mathrm{d} t\right] .
$$

We note that Assumption 4.2 (a) below guarantees that the negative part of the integrand in (2.15) is bounded, so that the expectation is well defined. The value of our hedging problem is

$$
v(\psi)=v(\psi ; \mathfrak{Y}, \mathfrak{P}):=\sup _{\boldsymbol{v} \in \mathfrak{Y}} \inf _{P \in \mathfrak{P}} J^{\psi}(\boldsymbol{v}, P) .
$$

To wit, the agent wants to find a strategy in $\mathfrak{Y}$ that maximises the numerical representation of her preferences (2.12). The goal of this paper is to find an asymptotic expansion of the value $v(\psi)$ for small levels of uncertainty aversion $\psi$ and to find a trading strategy that achieves the leading-order optimal performance.

\section{Heuristics}

The asymptotic solution of the family of SDGs (2.16) is related to a linearly constrained quadratic programming problem. In this section, we derive this optimisation problem heuristically from the HJBI equation associated to (2.16). This motivates the definitions of the functions introduced in the subsequent Section 4

Effective greeks. Let us assume for the moment that the true dynamics of the stock price are given by the Black-Scholes model with some (constant) volatility $\Sigma_{0}$. Then Itô's formula and the PDE (2.9) for $\mathcal{V}$ show that the replicating strategy (trading only the stock and the bank account, not the call) of the option with payoff $\mathrm{V}\left(S_{T}, A_{T}, M_{T}\right)$ is given by $\theta_{t}=\left(\mathcal{V}_{S}+\gamma \mathcal{V}_{A}\right)\left(t, S_{t}, A_{t}, M_{t}, \Sigma_{0}\right)$. In particular, the delta $\mathcal{V}_{S}$ of the option only gives the replicating strategy if $\gamma \equiv 0$, i.e., if the additional state variable $A$ is of finite variation (e.g., for vanilla options like the liquidly traded call, or exotics like barrier, Asian, or lookback options). In general, however, the replicating strategy also has to take into account the indirect sensitivity of the option value with respect to changes in the stock price arising from the additional state variable $A$ (e.g., for a forward-start option as in Example 3.1). Therefore, we call

$$
\Delta=\mathcal{V}_{S}+\gamma \mathcal{V}_{A}
$$

the effective delta of the option V. Similarly, we call

$$
\Gamma=\mathcal{V}_{S S}+2 \gamma \mathcal{V}_{S A}+\gamma^{2} \mathcal{V}_{A A} \quad \text { and } \quad \frac{\partial \Delta}{\partial \Sigma}=\mathcal{V}_{S \Sigma}+\gamma \mathcal{V}_{A \Sigma}
$$

the effective gamma and effective vanna of the option $\mathrm{V}$, respectively.

Example 3.1 (Forward-start call). A forward-start call with payoff $\left(S_{T}-S_{T_{\text {reset }}}\right)^{+}$is a call option whose strike is set at some future reset date $T_{\text {reset }} \in(0, T)$ (cf., e.g., 44, Section 6.2]). This option payoff can be embedded into our framework by choosing $A_{0}=S_{0}$ and $\gamma(t)=\mathbf{1}_{\left\{t<T_{\text {reset }}\right\}}$. Indeed, then $A_{t}=S_{t \wedge T_{\text {reset }}}$ and the option payoff can be written as $\mathrm{V}\left(S_{T}, A_{T}\right)=\left(S_{T}-A_{T}\right)^{+}$. 
Dynamics of the P\&L process. In order to write down the HJBI equation associated to the hedging problem, we need the dynamics of the P\&L process $Y^{\boldsymbol{v}}, P$ for generic strategies $\boldsymbol{v}$ and models $P$. Applying Itô's formula to $Y^{\boldsymbol{v}, P}$ (defined in (2.11) ) under $P$ (with associated control $\zeta^{P}$ ) yields (cf. Lemma 5.2)

$$
\begin{aligned}
\mathrm{d} Y_{t}^{\boldsymbol{v}, P}=( & \left.\theta_{t}-\left(\Delta\left(t, \mathbf{X}_{t}\right)-\phi_{t} \mathcal{C}_{S}\left(t, S_{t}, \Sigma_{t}\right)\right)\right) \mathrm{d} S_{t}+\left(\phi_{t} \mathcal{C}_{\Sigma}\left(t, S_{t}, \Sigma_{t}\right)-\mathcal{V}_{\Sigma}\left(t, \mathbf{X}_{t}\right)\right) \mathrm{d} \Sigma_{t}^{c, P} \\
& -b^{\mathcal{V}}\left(t, \mathbf{X}_{t} ; \boldsymbol{\zeta}_{t}^{P}\right) \mathrm{d} t
\end{aligned}
$$

where $\Sigma^{c, P}=\Sigma-\int_{0}^{.} \nu_{u}^{P} \mathrm{~d} u$ is the (continuous) local martingale part of $\Sigma$ under $P$, and (writing $\mathbf{x}=(S, A, M, \Sigma) \in \mathbb{R}^{4}$ and $\left.\boldsymbol{\zeta}=(\nu, \sigma, \eta, \xi) \in \mathbb{R}^{4}\right)$

$$
b^{\mathcal{V}}(t, \mathbf{x} ; \boldsymbol{\zeta})=\nu \mathcal{V}_{\Sigma}+\frac{1}{2}\left(\beta \mathcal{V}_{A}+S^{2} \Gamma\right)\left(\sigma^{2}-\Sigma^{2}\right)+\sigma \eta S \frac{\partial \Delta}{\partial \Sigma}+\frac{1}{2}\left(\eta^{2}+\xi\right) \mathcal{V}_{\Sigma \Sigma}
$$

For small uncertainty aversion, models far from the reference model are heavily penalised. Whence, the fictitious adversary needs to choose among small perturbations $\boldsymbol{\zeta}=\boldsymbol{\zeta}^{0}(\Sigma)+\widetilde{\boldsymbol{\zeta}} \psi$ of the reference feedback control $\zeta^{0}(\Sigma)=(0, \Sigma, 0,0)$. Plugging this perturbation into (3.1), we find

$$
b^{\mathcal{V}}(\boldsymbol{\zeta})=\mathbf{v}^{\top} \widetilde{\boldsymbol{\zeta}} \psi+o(\psi)
$$

where $\mathbf{v}=\left(\mathcal{V}_{\Sigma}, \Sigma\left(\beta \mathcal{V}_{A}+S^{2} \Gamma\right), \Sigma S \frac{\partial \Delta}{\partial \Sigma}, \frac{1}{2} \mathcal{V}_{\Sigma \Sigma}\right)$. Note that by expanding the function $b^{\mathcal{V}}\left(\boldsymbol{\zeta}^{0}(\Sigma)+\widetilde{\boldsymbol{\zeta}} \psi\right)$ around $\psi=0$, the vector-valued function $\mathbf{v}$ in (3.2) can also be identified as the gradient $\mathrm{D}_{\zeta} b^{\mathcal{V}}$ evaluated in $\zeta^{0}(\Sigma)$.

Remark 3.2. We now explain the use of the uncorrelated squared volatility of implied volatility $\xi$ as a control variable. Equation (3.2) shows that an $O(\psi)$-perturbation of the squared volatility around zero (i.e., a positive fourth component of $\widetilde{\boldsymbol{\zeta}}$ ) affects the drift $b^{\mathcal{V}}$ at the order $O(\psi)$ (at least as long as we are in the generic case where $\mathcal{V}_{\Sigma \Sigma}$ is nonzero). If we used instead the uncorrelated volatility $\xi^{\prime}:=\sqrt{\xi}$ as a basic control variable, then $\xi$ in (3.1) would be replaced by $\left(\xi^{\prime}\right)^{2}$. Following the arguments that lead to (3.2), we would then find for a perturbation of the form $\boldsymbol{\zeta}^{\prime}=\boldsymbol{\zeta}^{0}(\Sigma)+\widetilde{\boldsymbol{\zeta}}^{\prime} \psi$ that

$$
b^{\mathcal{V}}\left(\boldsymbol{\zeta}^{\prime}\right)=\left(\mathbf{v}^{\prime}\right)^{\top} \tilde{\boldsymbol{\zeta}}^{\prime} \psi+o(\psi)
$$

where $\mathbf{v}^{\prime}$ is given by $\mathbf{v}$ with the fourth component replaced by zero. Thus, a perturbation of $\xi^{\prime}$ around zero of order $O(\psi)$ would then have no impact on the $O(\psi)$ term in the expansion (3.3) of $b^{\mathcal{V}}$. This is an artefact of the Black-Scholes reference model: for any reference model with a nonzero uncorrelated volatility of implied volatility, $\xi^{\prime 0} \neq 0$, the fourth component of $\mathbf{v}^{\prime}$ would generically not vanish, and hence an $O(\psi)$-perturbation of $\xi^{\prime}$ around $\xi^{\prime 0}$ would affect the drift $b^{\mathcal{V}}$ at the order $O(\psi)$.

HJBI equation. The drift condition (2.7) can be rephrased as $b^{\mathcal{C}}\left(t, \mathbf{X}_{t} ; \boldsymbol{\zeta}_{t}^{P}\right)=0 \mathrm{~d} t \times P$-a.e., where

$$
b^{\mathcal{C}}(t, \mathbf{x} ; \boldsymbol{\zeta})=\nu \mathcal{C}_{\Sigma}+\frac{1}{2} S^{2} \mathcal{C}_{S S}\left(\sigma^{2}-\Sigma^{2}\right)+\sigma \eta S \mathcal{C}_{S \Sigma}+\frac{1}{2}\left(\eta^{2}+\xi\right) \mathcal{C}_{\Sigma \Sigma}
$$

In addition, the uncorrelated squared volatility of implied volatility $\xi^{P}$ (the fourth component of $\zeta^{P}$ ) must be nonnegative (cf. Definition 2.3). Hence, the HJBI equation associated to the hedging problem reads as

$$
w_{t}^{\psi}(t, \mathbf{x}, y)+\sup _{\boldsymbol{v} \in \mathbb{R}^{2}} \inf _{\substack{\boldsymbol{\zeta} \in \mathbb{R}^{4}: \\ b^{\mathcal{C}}(t, \mathbf{x} ; \boldsymbol{\zeta})=0, \zeta_{4} \geq 0}} H^{\psi}(t, \mathbf{x}, y ; \boldsymbol{v}, \boldsymbol{\zeta})=0, \quad w^{\psi}(T, \mathbf{x}, y)=U(y),
$$

where the function $H^{\psi}(t, \mathbf{x}, y ; \boldsymbol{v}, \boldsymbol{\zeta})$ (spelled out explicitly in (5.15)) depends on first- and secondorder partial derivatives of $w^{\psi}$ with respect to the space variables $\mathbf{x}$ and $y$, and on the drift and 
diffusion coefficients describing the dynamics of $S, A, M, \Sigma$, and $Y^{\boldsymbol{v}, P}$ under a model $P$ such that $\boldsymbol{\zeta}_{t}^{P}=\boldsymbol{\zeta}$. We refer to [28, Section 4.1] for a derivation of the HJBI equation from the martingale optimality principle of stochastic optimal control. In essence, the left-hand side of the HJBI equation arises from the drift of the process $w^{\psi}\left(t, \mathbf{X}_{t}, Y_{t}^{\boldsymbol{v}, P}\right)+\frac{1}{\psi} \int_{0}^{t} U^{\prime}\left(Y_{u}^{\boldsymbol{v}, P}\right) f\left(\Sigma_{u}, \boldsymbol{\zeta}_{u}^{P}\right) \mathrm{d} u$ under $P$, which can be computed via Itô's formula.

Asymptotic ansatz. As the Black-Scholes model is complete and the drift of the liquidly traded assets is zero under each model by assumption, we expect that the zeroth-order term in the expansion of $v(\psi)$ is simply the utility $U\left(Y_{0}\right)$ generated by the initial P\&L. Similarly, the optimal control $\zeta$ with zero uncertainty aversion should simply be the reference feedback control $\zeta^{0}(\Sigma)$. This motivates the following ansatz for the asymptotic expansion of the value function and the almost optimal feedback control 32

$$
\begin{aligned}
w^{\psi}(t, \mathbf{x}, y) & =U(y)-U^{\prime}(y) \widetilde{w}(t, \mathbf{x}) \psi \\
\boldsymbol{\zeta}^{\psi}(t, \mathbf{x}) & =\boldsymbol{\zeta}^{0}(\Sigma)+\widetilde{\boldsymbol{\zeta}}(t, \mathbf{x}) \psi
\end{aligned}
$$

for functions $\widetilde{w}$ and $\widetilde{\zeta}=(\widetilde{\nu}, \widetilde{\sigma}, \widetilde{\eta}, \widetilde{\xi})$ to be determined. In the reference model, any strategy in the stock and the call that neutralises the net delta qualifies as a replicating strategy. Whence, it is less obvious whether the delta-vega hedge $\boldsymbol{v}^{\star}$ or any other strategy that neutralises the agent's net delta (e.g., the standard delta hedge without trading in the call) should be the candidate strategy $\boldsymbol{v}=(\theta, \phi)$ for the hedging problem. Thus, we leave the choice of $\phi$ open for the moment and just assume that

$$
\theta=\Delta-\phi \mathcal{C}_{S}
$$

neutralises the (effective) net delta. Plugging (3.5)-3.7) into the HJBI equation (3.4) (using the explicit formula (5.15) for $H^{\psi}$ ), dropping the $\sup _{\boldsymbol{v}} \inf _{\boldsymbol{\zeta}}$ (we assume that the candidate strategy and control form a saddle point), using the expansion (3.2), and ordering by powers of $\psi$, we obtain

$$
\begin{aligned}
U^{\prime} \times\left(-\widetilde{w}_{t}-\left(\alpha+\frac{1}{2} \beta \Sigma^{2}\right) \widetilde{w}_{A}-\frac{1}{2} \Sigma^{2} S^{2}\left(\widetilde{w}_{S S}+2 \gamma \widetilde{w}_{S A}+\gamma^{2} \widetilde{w}_{A A}\right)\right. \\
\left.+\frac{1}{2} \widetilde{\boldsymbol{\zeta}}^{\top} \Psi^{-1} \widetilde{\boldsymbol{\zeta}}-\mathbf{v}^{\top} \widetilde{\boldsymbol{\zeta}}-\frac{1}{2} \widetilde{\xi}\left(\phi \mathcal{C}_{\Sigma}-\mathcal{V}_{\Sigma}\right)^{2} \frac{-U^{\prime \prime}}{U^{\prime}}\right) \psi+o(\psi)=0 .
\end{aligned}
$$

Moreover, the constraints in the minimisation part of the HJBI equation transform to

$$
\left(\widetilde{\nu} \mathcal{C}_{\Sigma}+\widetilde{\sigma} \Sigma S^{2} \mathcal{C}_{S S}+\widetilde{\eta} \Sigma S \mathcal{C}_{S \Sigma}+\widetilde{\xi} \frac{1}{2} \mathcal{C}_{\Sigma \Sigma}\right) \psi+o(\psi)=0 \quad \text { and } \quad \widetilde{\xi} \geq 0
$$

Our candidates for $\widetilde{\zeta}$ and $\phi$ now arise as the saddle point of the min-max problem (minimising over $\widetilde{\boldsymbol{\zeta}}$ and maximising over $\phi)$ corresponding to the $O(\psi)$ term in (3.8) subject to the constraints (3.9). Clearly, the vega hedge $\phi^{\star}=\frac{\mathcal{V}_{\Sigma}}{\mathcal{C}_{\Sigma}}$ maximises the $O(\psi)$ term over $\phi \in \mathbb{R}$, irrespective of the choice of $\widetilde{\zeta}$. With this choice, the constrained minimisation over $\widetilde{\zeta}$ (ignoring the $o(\psi)$ term in the equality constraint in (3.9) ) reduces to a linearly constrained quadratic programming problem:

$$
\text { minimise } \frac{1}{2} \widetilde{\boldsymbol{\zeta}}^{\top} \Psi^{-1} \widetilde{\boldsymbol{\zeta}}-\mathbf{v}^{\top} \widetilde{\boldsymbol{\zeta}} \quad \text { subject to } \widetilde{\boldsymbol{\zeta}} \in \mathbb{R}^{4}, \mathbf{c}^{\top} \widetilde{\boldsymbol{\zeta}}=0, \widetilde{\zeta}_{4} \geq 0
$$

where $\mathbf{c}=\left(\mathcal{C}_{\Sigma}, \Sigma S^{2} \mathcal{C}_{S S}, \Sigma S \mathcal{C}_{S \Sigma}, \frac{1}{2} \mathcal{C}_{\Sigma \Sigma}\right)$

\footnotetext{
${ }^{32}$ In view of [28, it is expected that $\psi$ (and not, e.g., $\psi^{1 / 2}$ or $\psi^{2}$ ) is the correct power for the expansion of the value function. Alternatively, one could write $\psi^{\alpha}$ instead of $\psi$ in (3.5) and then find $\alpha=1$ by matching the powers of the penalty term and the drift term of the P\&L process in the expansion of the HJBI equation in such a way that the optimisation over $\widetilde{\zeta}$ becomes nontrivial.
} 
Solving the linearly constrained quadratic program. The minimisation problem (3.10) is strictly convex and linearly constrained and thus has a unique minimum. The minimiser $\widetilde{\zeta}^{*}$ is characterised by the associated Karush-Kuhn-Tucker conditions

$$
\Psi^{-1} \widetilde{\boldsymbol{\zeta}}^{*}-\mathbf{v}+\lambda^{*} \mathbf{c}-\mu^{*} \overrightarrow{\mathbf{e}}_{4}=0, \quad \mathbf{c}^{\top} \widetilde{\boldsymbol{\zeta}}^{*}=0, \quad \widetilde{\zeta}_{4}^{*} \geq 0, \quad \mu^{*} \geq 0, \quad \mu^{*} \widetilde{\zeta}_{4}^{*}=0,
$$

for some scalars $\lambda^{*}$ and $\mu^{*}$. It turns out that there is an explicit solution $\left(\widetilde{\boldsymbol{\zeta}}^{*}, \lambda^{*}, \mu^{*}\right)$ (cf. Lemma A.1 (a)), which motivates our definitions in Section 4.1 .

\section{Main results}

This section contains the mathematically precise statement of our main results. In Section 4.1, we first introduce the required notation and technical assumptions; this notationally heavy part can be skipped at first reading.

\subsection{Notation and assumptions}

Our main result, Theorem 4.5, provides an asymptotic expansion of the value $v(\psi)$ from (2.16) for small levels of uncertainty aversion $\psi$ and an asymptotic saddle point $\left(\boldsymbol{v}^{\star}, P^{\psi}\right)_{\psi}$, where $\boldsymbol{v}^{\star}$ is the delta-vega hedge and $\left(P^{\psi}\right)_{\psi}$ is a suitable family of models. To define the PDE that describes the first-order term of the expansion and to define the quadruple $\boldsymbol{\zeta}^{\psi}$ that corresponds approximately (see Definition 4.1 (b) below) to $P^{\psi}$, we need to introduce some notation.

Recall that $\mathbf{G}=\mathbb{R}_{+} \times \mathbb{R} \times \mathbb{R}_{+}$is the state space of the process $(S, A, M)$ and set $\mathbf{D}^{0}=$ $(0, T) \times \mathbf{G} \times \mathbb{R}_{+}$. A generic element of $\mathbf{D}^{0}$ is written as $(t, S, A, M, \Sigma)$ or $(t, \mathbf{x})$ with $\mathbf{x}=(S, A, M, \Sigma)$. The functions $\Delta, \Gamma, \frac{\partial \Delta}{\partial \Sigma}: \mathbf{D}^{0} \rightarrow \mathbb{R}$ defined by 33

$$
\Delta(t, \mathbf{x})=\mathcal{V}_{S}+\gamma \mathcal{V}_{A}, \quad \Gamma(t, \mathbf{x})=\mathcal{V}_{S S}+2 \gamma \mathcal{V}_{S A}+\gamma^{2} \mathcal{V}_{A A}, \quad \frac{\partial \Delta}{\partial \Sigma}(t, \mathbf{x})=\mathcal{V}_{S \Sigma}+\gamma \mathcal{V}_{A \Sigma}
$$

are called the effective delta, effective gamma, and effective vanna of the option $\mathrm{V}$, respectively; we note that these quantities correspond to the standard greeks if $\gamma \equiv 0$ like for vanilla, barrier, or lookback options, for example, and refer to Section 3 for a motivation of this terminology in the case $\gamma \not \equiv 0$. The functions $\mathbf{c}, \mathbf{v}: \mathbf{D}^{0} \rightarrow \mathbb{R}^{4}$ given by

$$
\begin{aligned}
& \mathbf{c}(t, \mathbf{x})=\left(\mathcal{C}_{\Sigma}, \Sigma S^{2} \mathcal{C}_{S S}, \Sigma S \mathcal{C}_{S \Sigma}, \frac{1}{2} \mathcal{C}_{\Sigma \Sigma}\right)^{\top} \\
& \mathbf{v}(t, \mathbf{x})=\left(\mathcal{V}_{\Sigma}, \Sigma\left(\beta \mathcal{V}_{A}+S^{2} \Gamma\right), \Sigma S \frac{\partial \Delta}{\partial \Sigma}, \frac{1}{2} \mathcal{V}_{\Sigma \Sigma}\right)^{\top},
\end{aligned}
$$

are called the vega-gamma-vanna-volga vector of the call and the option $\mathrm{V}$, respectively. With this notation, define the functions $\lambda, \mu: \mathbf{D}^{0} \rightarrow \mathbb{R}$ and $\widetilde{\boldsymbol{\zeta}}: \mathbf{D}^{0} \rightarrow \mathbb{R}^{4}$ as follows:

$$
\begin{aligned}
& \lambda(t, \mathbf{x})= \begin{cases}\frac{\mathbf{c}^{\top} \Psi \mathbf{v}}{\mathbf{c}^{\top} \Psi \mathbf{c}} & \text { if } \mathcal{V}_{\Sigma \Sigma}-\frac{\mathbf{c}^{\top} \Psi \mathbf{v}}{\mathbf{c}^{\top} \Psi \mathbf{v}} \mathcal{C}_{\Sigma \Sigma} \geq 0, \\
\frac{\mathbf{c}^{\top} \Psi \mathbf{c}-\frac{1}{4} \mathcal{C}_{\Sigma \Sigma} \mathcal{V}_{\Sigma \Sigma} \mathcal{C}_{\Sigma} \psi_{\xi}}{\mathbf{c}_{\xi}} & \text { otherwise, }\end{cases} \\
& \mu(t, \mathbf{x})=\frac{1}{2}\left(\mathcal{V}_{\Sigma \Sigma}-\lambda \mathcal{C}_{\Sigma \Sigma}\right)^{-}, \\
& \widetilde{\boldsymbol{\zeta}}(t, \mathbf{x})=\Psi\left(\mathbf{v}-\lambda \mathbf{c}+\mu \overrightarrow{\mathbf{e}}_{4}\right) .
\end{aligned}
$$

Note that the term $\mu \overrightarrow{\mathbf{e}}_{4}$ in $(4.5)$ ensures that the fourth component of $\widetilde{\zeta}$ is nonnegative. Now, fix constants $0<\underline{\Sigma}<\Sigma_{0}<\bar{\Sigma}$, and define for each $\psi>0$, the candidate feedback control $\boldsymbol{\zeta}^{\psi}=$ $\left(\nu^{\psi}, \sigma^{\psi}, \eta^{\psi}, \xi^{\psi}\right): \mathbf{D}^{0} \rightarrow \mathbb{R}^{4}$ by

$$
\boldsymbol{\zeta}^{\psi}(t, \mathbf{x})=\boldsymbol{\zeta}^{0}(\Sigma)+\widetilde{\boldsymbol{\zeta}} \mathbf{1}_{\{\underline{\Sigma}<\Sigma<\bar{\Sigma}\}} \psi
$$

\footnotetext{
${ }^{33}$ Here and in the following, we assume that all relevant partial derivatives of $\mathcal{C}$ and $\mathcal{V}$ exist; precise conditions are given in Assumption 4.2 below.
} 
The indicator $\mathbf{1}_{\{\underline{\Sigma}<\Sigma<\bar{\Sigma}\}}$ is a technical modification that ensures that the implied volatility stays within the interval $[\underline{\Sigma}, \bar{\Sigma}]$ by falling back to the reference feedback control $\zeta^{0}(\Sigma)$ (which corresponds to constant implied volatility) as soon as the implied volatility hits the boundary of $[\underline{\Sigma}, \bar{\Sigma}]$. More explicitly, the candidate feedback control can be expressed as

$$
\begin{aligned}
\nu^{\psi}(t, \mathbf{x}) & =\left(\mathcal{V}_{\Sigma}-\lambda \mathcal{C}_{\Sigma}\right) \mathbf{1}_{\{\underline{\Sigma}<\Sigma<\bar{\Sigma}\}} \psi_{\nu} \psi, \\
\sigma^{\psi}(t, \mathbf{x}) & =\Sigma+\Sigma\left(\beta \mathcal{V}_{A}+S^{2} \Gamma-\lambda S^{2} \mathcal{C}_{S S}\right) \mathbf{1}_{\{\underline{\Sigma}<\Sigma<\bar{\Sigma}\}} \psi_{\sigma} \psi, \\
\eta^{\psi}(t, \mathbf{x}) & =\Sigma\left(S \frac{\partial \Delta}{\partial \Sigma}-\lambda S \mathcal{C}_{S \Sigma}\right) \mathbf{1}_{\{\underline{\Sigma}<\Sigma<\bar{\Sigma}\}} \psi_{\eta} \psi, \\
\xi^{\psi}(t, \mathbf{x}) & =\frac{1}{2}\left(\mathcal{V}_{\Sigma \Sigma}-\lambda \mathcal{C}_{\Sigma \Sigma}\right)^{+} \mathbf{1}_{\{\underline{\Sigma}<\Sigma<\bar{\Sigma}\}} \psi_{\xi} \psi
\end{aligned}
$$

In general, there is no $P^{\psi} \in \mathfrak{P}^{0}$ such that $\boldsymbol{\zeta}^{\psi}$ coincides with the control $\boldsymbol{\zeta}^{P^{\psi}}$ corresponding to $P^{\psi}$ as the process $\boldsymbol{\zeta}^{\psi}\left(t, \mathbf{X}_{t}\right)$ fulfils the drift condition (2.7) only at the order $O(\psi)$. However, to match the drift condition exactly, one can perturb $\zeta^{\psi}$ by a suitable, asymptotically small term. This motivates part (b) of the following definition.

Definition 4.1. Let $\mathfrak{P} \subset \mathfrak{P}^{0}$.

(a) For each $p \geq 1$, we denote by $L_{\mathfrak{P}}^{p}$ the vector space of Borel functions $K: \mathbf{D}^{0} \rightarrow \mathbb{R}$ satisfying

$$
\|K\|_{L_{\mathfrak{P}}^{p}}:=\sup _{P \in \mathfrak{P}} E^{P}\left[\int_{0}^{T}\left|K\left(t, \mathbf{X}_{t}\right)\right|^{p} \mathrm{~d} t\right]^{1 / p}<\infty .
$$

(b) A family $\left(P^{\psi}\right)_{\psi \in\left(0, \psi_{0}\right)} \subset \mathfrak{P}$ for some $\psi_{0} \in(0,1)$ is called a candidate asymptotic model family (in $\mathfrak{P}$ ) if there is $K_{0} \in L_{\mathfrak{P}}^{4}$ such that for all $\psi \in\left(0, \psi_{0}\right)$,

$$
\left|\boldsymbol{\zeta}_{t}^{P^{\psi}}-\boldsymbol{\zeta}^{\psi}\left(t, \mathbf{X}_{t}\right)\right| \leq K_{0}\left(t, \mathbf{X}_{t}\right) \psi^{2} \quad \mathrm{~d} t \times P^{\psi} \text {-a.e. }
$$

The crucial property of a candidate asymptotic model family formalised in Definition 4.1 (b) is that the control $\boldsymbol{\zeta}_{t}^{P^{\psi}}$ corresponding to $P^{\psi}$ is $O\left(\psi^{2}\right)$-close to the candidate control $\boldsymbol{\zeta}^{\psi}\left(t, \mathbf{X}_{t}\right)$.

The leading-order coefficient of the asymptotic expansion of $v(\psi)$ is given in terms of the solution to a linear second-order parabolic PDE with a source term. Specifically, for each $\Sigma \in[\underline{\Sigma}, \bar{\Sigma}]$, we consider the PDE

$$
\begin{array}{rlrl}
\widetilde{w}_{t}+\left(\alpha+\frac{1}{2} \beta \Sigma^{2}\right) \widetilde{w}_{A} & \\
+\frac{1}{2} \Sigma^{2} S^{2}\left(\widetilde{w}_{S S}+2 \gamma \widetilde{w}_{S A}+\gamma^{2} \widetilde{w}_{A A}\right)+\frac{1}{2} \widetilde{g}(\cdot, \Sigma)=0 & & \text { on }(0, T) \times \mathbf{G}, \\
\delta \widetilde{w}_{A}+\widetilde{w}_{M}=0 & & \text { on }\{(t, S, A, M): S \geq M\}, \\
\widetilde{w}(T, \cdot, \Sigma)=0 & & \text { on } \mathbf{G},
\end{array}
$$

where the source term $\widetilde{g}: \mathbf{D}^{0} \rightarrow \mathbb{R}$ is given by

$$
\widetilde{g}(t, \mathbf{x})=\mathbf{v}(t, \mathbf{x})^{\top} \widetilde{\boldsymbol{\zeta}}(t, \mathbf{x}) .
$$

We prove our main result under the following assumptions.

Assumption 4.2. Set $\mathbf{D}=(0, T) \times \mathbf{G} \times[\underline{\underline{\Sigma}}, \bar{\Sigma}] \subset \mathbf{D}^{0}$.

(a) Trading strategy set: There is a constant $K_{\mathfrak{Y}}>0$ such that for each trading strategy $\boldsymbol{v} \in \mathfrak{Y}$ and each $P \in \mathfrak{P}, Y^{\boldsymbol{v}, P}>-K_{\mathfrak{Y}} \mathrm{d} t \times P$-a.e. 
(b) Model set: $\mathfrak{P} \subset \mathfrak{P}^{0}$ contains a candidate asymptotic model family, a reference model, and there are constants $\underline{\nu}<0<\bar{\nu}, 0<\underline{\sigma}<\underline{\Sigma}, \bar{\Sigma}<\bar{\sigma}, \underline{\eta}<0<\bar{\eta}$, and $\bar{\xi}>0$ such that for each $P \in \mathfrak{P}$,

$$
\nu^{P} \in[\underline{\nu}, \bar{\nu}], \sigma^{P} \in[\underline{\sigma}, \bar{\sigma}], \eta^{P} \in[\underline{\eta}, \bar{\eta}], \xi^{P} \in[0, \bar{\xi}], \Sigma \in[\underline{\Sigma}, \bar{\Sigma}] \quad \mathrm{d} t \times P \text {-a.e. }
$$

(c) Call PDE: $T_{\mathrm{C}} \geq T$ and there is $\mathcal{C} \in C^{1,2,2}\left(\left(0, T_{\mathrm{C}}\right) \times \mathbb{R}_{+} \times \mathbb{R}_{+}\right) \cap C\left(\left[0, T_{\mathrm{C}}\right] \times \overline{\mathbb{R}}_{+} \times \overline{\mathbb{R}}_{+}\right)$such that for each $\Sigma \in[\underline{\Sigma}, \bar{\Sigma}], \mathcal{C}(\cdot, \Sigma)$ is a classical solution to the PDE (2.1) and

$$
\mathcal{C}_{\Sigma} \neq 0 \quad \text { and } \quad\left|\mathcal{C}_{\Sigma \Sigma}\right| \leq K_{\mathcal{C}}\left(\left|\mathcal{C}_{\Sigma}\right|+\left|S^{2} \mathcal{C}_{S S}\right|+\left|S \mathcal{C}_{S \Sigma}\right|\right) \text { on }(0, T) \times \mathbb{R}_{+} \times[\underline{\Sigma}, \bar{\Sigma}]
$$
for some $K_{\mathcal{C}} \in L_{\mathfrak{P}}^{2}$.

(d) Non-traded option PDE: There is $\mathcal{V} \in C^{1,2,2,1,2}\left(\mathbf{D}^{0}\right) \cap C\left(\overline{\mathbf{D}^{0}}\right)$ such that for each $\Sigma \in[\underline{\Sigma}, \bar{\Sigma}]$, $\mathcal{V}(\cdot, \Sigma)$ is a classical solution to the PDE (2.9) with

$$
\left|\mathcal{V}_{\Sigma}\right|,\left|\beta \mathcal{V}_{A}+S^{2}\left(\mathcal{V}_{S S}+2 \gamma \mathcal{V}_{S A}+\gamma^{2} \mathcal{V}_{A A}\right)\right|,\left|S\left(\mathcal{V}_{S \Sigma}+\gamma \mathcal{V}_{A \Sigma}\right)\right|,\left|\mathcal{V}_{\Sigma \Sigma}\right| \leq K_{\mathcal{V}} \text { on } \mathbf{D}
$$

for some constant $K_{\mathcal{V}}>0$.

(e) Cash equivalent PDE: There is $\widetilde{w} \in C^{1,2,2,1,2}\left(\mathbf{D}^{0}\right) \cap C\left(\overline{\mathbf{D}^{0}}\right)$ such that for each $\Sigma \in[\underline{\Sigma}, \bar{\Sigma}]$, $\widetilde{w}(\cdot, \Sigma)$ is a classical solution to the PDE (4.7),

$$
0 \leq \widetilde{w} \leq K_{\widetilde{w}} \text { on } \mathbf{D}
$$

for some constant $K_{\widetilde{w}}>0$, and

$$
\widetilde{w}_{\Sigma}, S\left(\widetilde{w}_{S}+\gamma \widetilde{w}_{A}\right), \beta \widetilde{w}_{A}+S^{2}\left(\widetilde{w}_{S S}+2 \gamma \widetilde{w}_{S A}+\gamma^{2} \widetilde{w}_{A A}\right), S\left(\widetilde{w}_{S \Sigma}+\gamma \widetilde{w}_{A \Sigma}\right), \widetilde{w}_{\Sigma \Sigma} \in L_{\mathfrak{P}}^{4} .
$$

(f) Utility function: $U: \mathbb{R} \rightarrow \mathbb{R}$ is $C^{3}$ with $U^{\prime}>0, U^{\prime \prime}<0$ everywhere and has decreasing absolute risk aversion, i.e., $y \mapsto-\frac{U^{\prime \prime}(y)}{U^{\prime}(y)}$ is nonincreasing on $\mathbb{R}$.

Remark 4.3. Let us discuss the various requirements in Assumption 4.2.

(a) This constraint on the agent's credit line is an admissibility condition for the set of trading strategies. The P\&L process $Y^{\boldsymbol{v}, P}$ is required to be bounded from below, uniformly over all strategies in $\mathfrak{Y}$ and all models in $\mathfrak{P}$. We show in Corollary 5.3 that this is satisfied for the P\&L process associated to the delta-vega hedge $\boldsymbol{v}^{\star}$ (cf. (4.13)). Hence, making the constant $K_{\mathfrak{Y}}$ larger if necessary, the delta-vega hedge can always be added to the set of strategies $\mathfrak{Y}$.

(b) A construction of a candidate asymptotic model family compatible with (4.9) is outlined in Section 4.3. The existence of uniform bounds on the controls as well as the implied volatility are essential for various steps in the proof of the main result. This is not as big an assumption is it might appear at first glance. Indeed, as the conclusions of our main result do not depend on the choice of these bounds, they can be chosen arbitrarily large.

(c) These regularity assumptions ensure that $\mathcal{C}$ corresponds to the Black-Scholes value of the liquidly traded call. The condition $\mathcal{C}_{\Sigma} \neq 0$ guarantees that the delta-vega hedge (cf. (4.13)) is well defined. The second condition in (4.10) ensures that the volga of the call is dominated by the sum of its vega, cash gamma, and cash vanna.

For a plain-vanilla call option with payoff $\mathrm{C}(S)=(S-K)^{+}$, explicit formulas for these greeks show that this requirement is met if $\log S \in L_{\mathfrak{P}}^{2}$. This in turn follows easily from the explicit representation of $S$ as a stochastic exponential together with the boundedness of the spot volatility from Assumption 4.2 (b).

Another example is the log-contract with payoff $\mathrm{C}(S)=\log (S)$, for which $\mathcal{C}(t, S, \Sigma)=$ $\log (S)-\frac{1}{2} \Sigma^{2}\left(T_{\mathrm{C}}-t\right)$. Computing the relevant greeks shows that (4.10) holds in this case, too. Moreover, if $T_{\mathrm{C}}>T$, then even the stronger condition (4.17) of Lemma 4.9] is satisfied. 
(d) This is a regularity assumption on the option $\mathrm{V}$ similar to (c) However, we additionally enforce the bounds (4.11) to ensure that the vega-gamma-vanna-volga vector $\mathbf{v}$ is bounded. This assumption is satisfied if the option payoff $\mathrm{V}$ is sufficiently regular.

For example, consider the case where the payoff function $\mathrm{V}(S, A, M)=H(S)$ only depends on the stock price $S$. The corresponding Black-Scholes value can be written as

$$
\mathcal{V}(t, S, A, M, \Sigma)=\int_{-\infty}^{\infty} H\left(S \exp \left(\Sigma \sqrt{T-t} x-\frac{1}{2} \Sigma^{2}(T-t)\right)\right) \phi(x) \mathrm{d} x,
$$

where $\phi$ is the density function of the standard normal distribution. If the "terminal cash delta" $y H^{\prime}(y)$ and the "terminal cash gamma" $y^{2} H^{\prime \prime}(y)$ are bounded in $y \in \mathbb{R}_{+} 34$ then using dominated convergence to differentiate under the integral sign shows that $\mathcal{V}$ indeed satisfies Assumption 4.2 (d)

For exotic options, one can argue along the same lines. For example, for a lookback option with sufficiently regular payoff $\mathrm{V}(S, A, M)=H(S, M)$ (recall that $M$ is the variable for the running maximum of the stock), one can again verify that the probabilistic representation of its Black-Scholes value solves the the PDE (2.9) and inherits the required regularity of Assumption 4.2 (d) from the regularity of the payoff function $H$.

(e) This assumption posits that a (classical) solution $\widetilde{w}$ to the PDE (4.7) exists and satisfies certain bounds. The validity of this assumption depends on the regularity of the input quantities $\mathcal{C}, \mathcal{V}, \alpha, \beta, \gamma$, and $\delta$, and can be checked along the lines of (d) above 35

(f) It is not essential that the utility function is defined on the whole real line. In fact, as we only consider strategies such that the P\&L process is bounded from below by $-K_{\mathfrak{Y}}$ uniformly over trading strategies and models, we could also work with a (suitably displaced) utility function on $\mathbb{R}_{+}$. Also note that power and exponential utilities both have decreasing absolute risk aversion.

Remark 4.4. As long as the traded option is regular enough, there are many models which fulfil assumption (4.9) for the coefficients of the implied volatility dynamics. For instance, consider a stochastic volatility model of the form

$$
\begin{aligned}
& \mathrm{d} S_{t}=S_{t} a\left(Y_{t}\right) \mathrm{d} W_{t}^{0}, \\
& \mathrm{~d} Y_{t}=b\left(Y_{t}\right) \mathrm{d} t+c_{0}\left(Y_{t}\right) \mathrm{d} W_{t}^{0}+c_{1}\left(Y_{t}\right) \mathrm{d} W_{t}^{1},
\end{aligned}
$$

where the functions $a, b, c_{0}, c_{1}$ as well as their derivatives are all Lipschitz and bounded, and $a, c_{0}, c_{1}$ are in addition positive and bounded away from zero. Then the spot volatility $\sigma_{t}=a\left(Y_{t}\right)$ evolves in some bounded interval $[\underline{\sigma}, \bar{\sigma}]$. Now, let $\mathcal{C}^{\text {sv }}\left(t, S_{t}, Y_{t}\right)$ be the value of a log-contract with payoff $\log \left(S_{T_{\mathrm{C}}}\right)$ for some $T_{\mathrm{C}}>T$ computed in this stochastic volatility model (under some pricing measure). As the spot volatility $\sigma_{t}$ is bounded from above and from below, the value $\mathcal{C}^{\text {sv }}$ of the log-contract can be bounded from above and below by its Black-Scholes values for volatility $\underline{\sigma}$ and $\bar{\sigma}$, respectively. Whence, the implied volatility $\Sigma_{t}$ is uniformly bounded and bounded away from zero, too. To determine its drift and diffusion coefficients $\nu_{t}, \eta_{t}$, and $\xi_{t}$, apply Itô's formula on both sides of the equation $\mathcal{C}\left(t, S_{t}, \Sigma_{t}\right)=\mathcal{C}^{\mathrm{sv}}\left(t, S_{t}, Y_{t}\right)$ that defines $\Sigma_{t}$ and compare the coefficients of the $\mathrm{d} W^{0}$ - and $\mathrm{d} W^{1}$-terms. Using also that the cash delta for the log-contract is $S \mathcal{C}_{S}=S \mathcal{C}_{S}^{\mathrm{sv}}=1$, this leads to

$$
\eta_{t}=c_{0}\left(Y_{t}\right) \frac{\mathcal{C}_{Y}^{\mathrm{sv}}\left(t, S_{t}, Y_{t}\right)}{\mathcal{C}_{\Sigma}\left(t, S_{t}, \Sigma_{t}\right)}, \quad \xi_{t}=\left(c_{1}\left(Y_{t}\right) \frac{\mathcal{C}_{Y}^{\mathrm{sv}}\left(t, S_{t}, Y_{t}\right)}{\mathcal{C}_{\Sigma}\left(t, S_{t}, \Sigma_{t}\right)}\right)^{2}
$$

Now, differentiating the PDE for $\mathcal{C}^{\text {sv }}$ yields a PDE for its partial derivative $\mathcal{C}_{Y}^{\text {sv }}$ whose probabilistic representation shows that $\mathcal{C}_{Y}^{\text {sv }}$ is bounded. As $\mathcal{C}_{\Sigma}$ is uniformly bounded away from zero for the

\footnotetext{
${ }^{34}$ This holds, e.g., for a "smooth put", whose payoff is the Black-Scholes put value with some arbitrarily short maturity.

${ }^{35}$ See also [28, Remark 3.2] for a discussion of such regularity assumptions in a similar setting.
} 
log-contract with $T_{\mathrm{C}}>T, \eta$ and $\xi$ are uniformly bounded as well. Finally, by the drift condition (2.7) (which holds automatically here because $\mathcal{C}^{\mathrm{sv}}\left(t, S_{t}, Y_{t}\right)$ is a local martingale by construction), it follows that also the drift coefficient $\nu_{t}$ of the implied volatility is uniformly bounded. In summary, the market model derived from the stochastic volatility model (4.12) fulfils (4.9).

\subsection{Main result}

We are now in a position to state our main result, which provides an asymptotic expansion of the value in (2.16) and a corresponding asymptotically optimal policy. The existence of a suitable corresponding model set $\mathfrak{P}$ and a candidate asymptotic model family is considered in Section 4.3 below. Recall from Remark 4.3 (a) that the delta-vega hedge $\boldsymbol{v}^{\star}$ can always be included into the set of trading strategies $\mathfrak{Y}$ by making the constant $K_{\mathfrak{Y}}$ from Assumption 4.2 (a) larger if necessary.

The number $\widetilde{w}_{0}:=\widetilde{w}\left(0, \mathbf{X}_{0}\right)$ defined through the solution $\widetilde{w}$ to the PDE (4.7) determines the leading-order coefficient in the expansion of the value $v(\psi)$. As it also describes the (normalised) premium that the agent demands as a compensation for exposing herself to model misspecification (cf. the expansion (4.16) of the indifference ask price below), we call it the cash equivalent (of small uncertainty aversion).

Theorem 4.5. Let $\mathfrak{Y}$ be a set of trading strategies, $\mathfrak{P} \subset \mathfrak{P}^{0}$ a model set, and suppose that Assumption 4.2 is satisfied. Define the delta-vega hedging strategy $\boldsymbol{v}^{\star}=\left(\theta_{t}^{\star}, \phi_{t}^{\star}\right)_{t \in[0, T]}$ by

$$
\begin{aligned}
\theta_{t}^{\star} & =\left(\Delta-\frac{\mathcal{V}_{\Sigma}}{\mathcal{C}_{\Sigma}} \mathcal{C}_{S}\right)\left(t, S_{t}, A_{t}, M_{t}, \Sigma_{t}\right), \\
\phi_{t}^{\star} & =\frac{\mathcal{V}_{\Sigma}}{\mathcal{C}_{\Sigma}}\left(t, S_{t}, A_{t}, M_{t}, \Sigma_{t}\right) .
\end{aligned}
$$

If $\boldsymbol{v}^{\star} \in \mathfrak{Y}$ and $\left(P^{\psi}\right)_{\psi \in\left(0, \psi_{0}\right)} \subset \mathfrak{P}$ is a candidate asymptotic model family, then as $\psi \downarrow 0$ :

$$
\begin{aligned}
v(\psi) & =\sup _{\boldsymbol{v} \in \mathfrak{Y}} \inf _{P \in \mathfrak{P}} J^{\psi}(\boldsymbol{v}, P)=\inf _{P \in \mathfrak{P}} \sup _{\boldsymbol{v} \in \mathfrak{Y}} J^{\psi}(\boldsymbol{v}, P)+o(\psi) \\
& =J^{\psi}\left(\boldsymbol{v}^{\star}, P^{\psi}\right)+o(\psi)=\sup _{\boldsymbol{v} \in \mathfrak{Y}} J^{\psi}\left(\boldsymbol{v}, P^{\psi}\right)+o(\psi)=\inf _{P \in \mathfrak{P}} J^{\psi}\left(\boldsymbol{v}^{\star}, P\right)+o(\psi) \\
& =U\left(Y_{0}\right)-U^{\prime}\left(Y_{0}\right) \widetilde{w}_{0} \psi+o(\psi) .
\end{aligned}
$$

In particular, the delta-vega hedge $\boldsymbol{v}^{\star}$ is an optimal strategy at the leading order $O(\psi)$ among all strategies in $\mathfrak{Y}$, and $P^{\psi}$ is a leading-order optimal choice of model for the fictitious adversary among all models in $\mathfrak{P}$.

The lengthy proof of Theorem 4.5 is postponed to Section 5.1] The first-order term in the expansion of $v(\psi)$ in (4.14) is determined by the cash equivalent $\widetilde{w}_{0}$. Its probabilistic representation allows to identify the main factors that determine an option's susceptibility to model misspecification:

Proposition 4.6 (Feynman-Kac representation). Suppose that Assumption 4.2 holds and let $P^{0} \in \mathfrak{P}$ be a reference model. Then

$$
\widetilde{w}_{0}=\frac{1}{2} E^{P^{0}}\left[\int_{0}^{T} \widetilde{g}\left(t, S_{t}, A_{t}, M_{t}, \Sigma_{0}\right) \mathrm{d} t\right] .
$$

Here, the function $\widetilde{g}$ (defined in (4.8)) can be written as

$$
\begin{aligned}
& \widetilde{g}(t, S, A, M, \Sigma) \\
& \quad=-\Sigma\left(\phi^{\star} S^{2} \mathcal{C}_{S S}-\left(\beta \mathcal{V}_{A}+S^{2} \Gamma\right)\right) \widetilde{\sigma}-\Sigma\left(\phi^{\star} S \mathcal{C}_{S \Sigma}-S \frac{\partial \Delta}{\partial \Sigma}\right) \widetilde{\eta}-\frac{1}{2}\left(\phi^{\star} \mathcal{C}_{\Sigma \Sigma}-\mathcal{V}_{\Sigma \Sigma}\right) \widetilde{\xi},
\end{aligned}
$$

where the functions $(\widetilde{\nu}, \widetilde{\sigma}, \widetilde{\eta}, \widetilde{\xi})=\widetilde{\zeta}$ are defined in (4.5) and $\phi^{\star}=\frac{\mathcal{\nu}_{\Sigma}}{\mathcal{C}_{\Sigma}}$ is the vega hedge from Theorem 4.5. 
Proof. The Feynman-Kac representation is proved in Proposition 5.1 (also note that $\Sigma_{t}=\Sigma_{0}$ $\mathrm{d} t \times P^{0}$-a.e. because $P^{0}$ is a reference model). The representation of $\widetilde{g}$ is the content of Corollary 5.7 .

For an interpretation of this representation in the case of $\beta \equiv \gamma \equiv 0$, we refer to the discussion after equation (1.6) in the introduction. If $\gamma \neq \equiv 0$ (e.g., for a forward-start call as in Example 3.1), then the effective gamma and effective vanna are in general different from the gamma and vanna of the option. If the option $\mathrm{V}$ depends on the realised variance of the stock (e.g., a call on the realised variance), then a term $\beta \mathcal{V}_{A}$ is added to the effective gamma in (4.15).

The next proposition implies that whenever the vega-gamma-vanna-volga vectors of the call and the non-traded option $\mathrm{V}$ are collinear, the local impact of uncertainty aversion vanishes at the leading order.

Proposition 4.7. Fix $(t, \mathbf{x}) \in \mathbf{D}^{0}$. If the vega-gamma-vanna-volga vectors $\mathbf{c}(t, \mathbf{x})$ and $\mathbf{v}(t, \mathbf{x})$ are collinear, then $\widetilde{g}(t, \mathbf{x})=0$.

Proof. Fix $(t, \mathbf{x}) \in \mathbf{D}^{0}$ and let $k \in \mathbb{R}$ such that $\mathbf{v}(t, \mathbf{x})=k \mathbf{c}(t, \mathbf{x})$. Then by construction (cf. (4.3)(4.5) $), \lambda(t, \mathbf{x})=k, \mu(t, \mathbf{x})=0$, and $\widetilde{\boldsymbol{\zeta}}(t, \mathbf{x})=0$. Thus, $\widetilde{g}(t, \mathbf{x})=0$.

For example, consider the case where the non-traded option is a put with the same strike and maturity as the liquidly traded call. Then the put-call parity implies that the vegas, gammas, vannas, and volgas of both options coincide everywhere. Thus, $\widetilde{g} \equiv 0$ and hence the cash equivalent $\widetilde{w}_{0}$ vanishes. This is expected as put-call parity also provides a model-free hedge for this situation.

Indifference prices. The indifference ask price (for the non-traded option $\mathrm{V}$ ) is the price at which the agent is indifferent between keeping a flat position and changing her position by selling the non-traded option for that price.

Recall that $V_{0}$ is the initial reference value of the non-traded option $\mathrm{V}$ and that $\widetilde{w}_{0}$ is its cash equivalent. Let $v(y ; \psi)$ denote the value of our hedging problem corresponding to initial P\&L $y$. If the agent decides to sell the non-traded option for a price $p_{a}(\psi)$, then her initial $\mathrm{P} \& \mathrm{~L}$ for the hedging problem is $Y_{0}+p_{a}(\psi)-V_{0}$. Therefore, the equation determining the indifference ask price $p_{a}(\psi)$ reads as follows:

$$
U\left(Y_{0}\right)=v\left(Y_{0}+p_{a}(\psi)-V_{0} ; \psi\right)
$$

Using the expansion of $v$ from Theorem 4.5, straightforward computations yield

$$
p_{a}(\psi)=V_{0}+\widetilde{w}_{0} \psi+o(\psi) .
$$

Therefore, $\widetilde{w}_{0} \psi$ is the leading-order premium demanded by the agent as a compensation for exposing herself to model uncertainty.

Remark 4.8. Buying an option is the same as selling the negative of that option. However, the cash equivalents corresponding to $\mathrm{V}$ and $-\mathrm{V}$ are in general different. This asymmetry is caused by the constraint that the uncorrelated squared volatility must be nonnegative and the fact that the reference model has zero uncorrelated squared volatility. In other words, the uncorrelated squared volatility can only depart from its reference value in one direction. In contrast, the other control variables can deviate from their reference value in both directions.

\subsection{On the existence of a candidate asymptotic model family}

Our main result, Theorem 4.5, assumes that the set of models $\mathfrak{P}$ contains a candidate asymptotic model family. In this section, we prescribe a set of models $\mathfrak{P}$ and sketch the construction of a candidate asymptotic model family in $\mathfrak{P}$. Fix constants $0<\underline{\Sigma}<\Sigma_{0}<\bar{\Sigma}, \underline{\nu}<0<\bar{\nu}, 0<\underline{\sigma}<\underline{\Sigma}$, $\bar{\Sigma}<\bar{\sigma}, \underline{\eta}<0<\bar{\eta}$, and $\bar{\xi}>0$, and let $\mathfrak{P}$ denote the subset of models $P$ in $\mathfrak{P}^{0}$ such that the bounds (4.9) are satisfied. Under some further regularity assumptions on the greeks of the liquid option, $\mathfrak{P}$ then contains a candidate asymptotic model family. 
The construction of the candidate asymptotic model family comprises two steps. The first is to prove that the candidate feedback control $\boldsymbol{\zeta}^{\psi}$ can be modified by a term of order $O\left(\psi^{2}\right)$ such that the resulting modified feedback control $\check{\zeta}^{\psi}$ satisfies the drift condition (2.7):

Lemma 4.9. Let $\mathfrak{P} \subset \mathfrak{P}^{0}$ be such that (4.9) holds for every $P \in \mathfrak{P}$. Suppose in addition that Assumption 4.2 (c) (d) holds with (4.10) in Assumption 4.2 (c) replaced by the stronger condition that

$$
\left|\mathcal{C}_{\Sigma}\right| \geq 1 / K_{\mathcal{C}} \quad \text { and } \quad\left|S^{2} \mathcal{C}_{S S}\right|,\left|S \mathcal{C}_{S \Sigma}\right|,\left|\mathcal{C}_{\Sigma \Sigma}\right| \leq K_{\mathcal{C}} \text { on }(0, T) \times \mathbb{R}_{+} \times[\underline{\Sigma}, \bar{\Sigma}]
$$

for some constant $K_{\mathcal{C}}>0$. Then there are $\psi_{0}>0$ and functions

$$
\check{\zeta}^{\psi}: \mathbf{D}^{0} \rightarrow[\underline{\nu}, \bar{\nu}] \times[\underline{\sigma}, \bar{\sigma}] \times[\underline{\eta}, \bar{\eta}] \times[0, \bar{\xi}], \quad \psi \in\left(0, \psi_{0}\right),
$$

such that for each $\psi \in\left(0, \psi_{0}\right)$, the restriction $\left.\check{\zeta}^{\psi}\right|_{(0, T) \times \mathbf{G} \times(\Sigma, \bar{\Sigma})}$ is continuous and can be extended to a continuous function on $\mathbf{D}^{0}=(0, T) \times \mathbf{G} \times \mathbb{R}_{+}$. Moreover, there is $K_{0}>0$ such that for each $(t, \mathbf{x})=(t, S, A, M, \Sigma) \in \mathbf{D}$ and $\psi \in\left(0, \psi_{0}\right)$,

(a) $\check{\zeta}^{\psi}(t, \mathbf{x})=\zeta^{0}(\Sigma)$ if $\Sigma \notin(\underline{\Sigma}, \bar{\Sigma})$, i.e., the modified feedback control falls back to the reference feedback control if the bounds on the implied volatility are reached;

(b) writing $\left(\check{\nu}^{\psi}, \check{\sigma}^{\psi}, \check{\eta}^{\psi}, \check{\xi}^{\psi}\right)=\check{\zeta}^{\psi}(t, \mathbf{x})$, we have

$$
\check{\nu}^{\psi} \mathcal{C}_{\Sigma}+\frac{1}{2} S^{2} \mathcal{C}_{S S}\left(\left(\check{\sigma}^{\psi}\right)^{2}-\Sigma^{2}\right)+\check{\sigma}^{\psi} \check{\eta}^{\psi} S \mathcal{C}_{S \Sigma}+\frac{1}{2}\left(\left(\check{\eta}^{\psi}\right)^{2}+\check{\xi}^{\psi}\right) \mathcal{C}_{\Sigma \Sigma}=0,
$$

i.e., the drift condition (2.7) is satisfied for the modified feedback control $\check{\zeta}^{\psi}$;

(c)

$$
\left|\check{\zeta}^{\psi}(t, \mathbf{x})-\zeta^{\psi}(t, \mathbf{x})\right| \leq K_{0} \psi^{2}
$$

i.e., the modified feedback control $\check{\zeta}^{\psi}$ is $O\left(\psi^{2}\right)$-close to the candidate $\boldsymbol{\zeta}^{\psi}$.

Proof. See Section 5.2

Let $\psi_{0}, K_{0}$, and $\check{\zeta}^{\psi}$ be as in Lemma 4.9. The second step now is to show that the stochastic differential equations (SDEs) corresponding to the modified feedback control $\check{\zeta}^{\psi}=\left(\check{\nu}^{\psi}, \check{\sigma}^{\psi}, \check{\eta}^{\psi}, \check{\xi}^{\psi}\right)^{\top}$ have a weak solution. Fix $\psi \in\left(0, \psi_{0}\right)$. Writing $\nu, \sigma, \eta, \xi$ instead of $\check{\nu}^{\psi}, \check{\sigma}^{\psi}, \check{\eta}^{\psi}, \check{\xi}^{\psi}$ to ease the notation, the relevant SDEs read as

$$
\begin{aligned}
\mathrm{d} S_{t}^{\prime} & =S_{t}^{\prime} \sigma \mathrm{d} W_{t}^{0}, \\
\mathrm{~d} \Sigma_{t}^{\prime} & =\nu \mathrm{d} t+\eta \mathrm{d} W_{t}^{0}+\sqrt{\xi} \mathrm{d} W_{t}^{1}, \\
\mathrm{~d} A_{t}^{\prime} & =\left(\alpha+\frac{1}{2} \beta \sigma^{2}\right) \mathrm{d} t+\gamma S_{t}^{\prime} \sigma \mathrm{d} W_{t}^{0}+\delta \mathrm{d} M_{t}^{\prime},
\end{aligned}
$$

where $\alpha, \beta, \gamma$, and $\delta$ are evaluated at $\left(t, S_{t}^{\prime}, A_{t}^{\prime}, M_{t}^{\prime}:=\sup _{u \in[0, t]} S_{u}^{\prime}\right), \nu, \sigma, \eta$, and $\xi$ are evaluated at $\left(t, S_{t}^{\prime}, A_{t}^{\prime}, M_{t}^{\prime}, \Sigma_{t}^{\prime}\right)$, and $\left(W^{0}, W^{1}\right)$ is a bivariate standard Brownian motion.

Suppose there exists a weak solution to (4.19) (starting in $S_{0}, \Sigma_{0}, A_{0}$ ) with the property that $\Sigma^{\prime}$ evolves in $[\underline{\Sigma}, \bar{\Sigma}]$ almost surely and denote by $P^{\psi}$ its image measure (under $\left(S^{\prime}, \Sigma^{\prime}, A^{\prime}\right)$ ) on the canonical space $(\Omega, \mathcal{F})$. Then by construction (cf. Definitions 2.3 and 2.5$), P^{\psi} \in \mathfrak{P}^{0}$ and $\boldsymbol{\zeta}_{t}^{P^{\psi}}=\check{\zeta}^{\psi}\left(t, \mathbf{X}_{t}\right) \mathrm{d} t \times P^{\psi}$-a.e. Moreover, by Lemma 4.9 and the fact that under $P^{\psi}, \Sigma$ evolves in $[\underline{\Sigma}, \bar{\Sigma}]$ almost surely, (4.9) holds for every $P \in\left(P^{\psi}\right)_{\psi \in\left(0, \psi_{0}\right)}$ and

$$
\left|\boldsymbol{\zeta}_{t}^{P^{\psi}}-\boldsymbol{\zeta}^{\psi}\left(t, \mathbf{X}_{t}\right)\right|=\left|\check{\boldsymbol{\zeta}}^{\psi}\left(t, \mathbf{X}_{t}\right)-\boldsymbol{\zeta}^{\psi}\left(t, \mathbf{X}_{t}\right)\right| \leq K_{0} \psi^{2} \quad \mathrm{~d} t \times P^{\psi} \text {-a.e. }
$$


So $\left(P^{\psi}\right)_{\psi \in\left(0, \psi_{0}\right)}$ is a candidate asymptotic model family in $\mathfrak{P}$.

It remains to argue the existence of a weak solution to (4.19) with the property that $\Sigma^{\prime}$ evolves in $[\underline{\Sigma}, \bar{\Sigma}]$. Note that we cannot directly apply standard existence results for weak solutions as the control $\check{\zeta}^{\psi}$ is not continuous in $\Sigma \in \mathbb{R}_{+}$. However, one can apply a standard existence result to the SDEs corresponding to the continuous extension of $\left.\check{\zeta}^{\psi}\right|_{(0, T) \times \mathbf{G} \times(\underline{\Sigma}, \bar{\Sigma})}$ to $\mathbf{D}^{0}$. Then the obvious idea is to stop the resulting weak solution as soon as $\Sigma^{\prime}$ hits the boundary of $[\underline{\Sigma}, \bar{\Sigma}]$ and restart the SDEs with new dynamics from there. After the restart, we keep $\Sigma^{\prime} \in\{\underline{\Sigma}, \bar{\Sigma}\}$ constant, let $S^{\prime}$ evolve like a standard Black-Scholes model with constant volatility $\Sigma^{\prime}$, and (assuming suitable Lipschitz and linear growth conditions on the coefficients of the SDE for $A$; cf. [28, Appendix B]) find a solution $A^{\prime}$ according to the dynamics in (4.19), but with the new dynamics of $S^{\prime}$. Then one can check that the constructed process satisfies the SDEs (4.19) with the original feedback control $\breve{\zeta}^{\psi}$; see [28, Theorem 3.7] for more details in a similar setup.

\section{Proofs}

This section contains the proofs of our main results. We first establish the value expansion and almost-optimality of the delta-vega hedge asserted in Theorem 4.5. Afterwards, we turn to the construction of the modified feedback control from Lemma 4.9.

\subsection{Value expansion and almost optimality of the delta-vega hedge}

In this section, we prove Theorem 4.5. Throughout, we assume that Assumption 4.2 is in force, that $\boldsymbol{v}^{\star} \in \mathfrak{Y}$, and that $\left(P^{\psi}\right)_{\psi \in\left(0, \psi_{0}\right)} \subset \mathfrak{P}$ is a candidate asymptotic model family 36 In particular (recall Definition 4.1 (b)), we fix $1 \leq K_{0} \in L_{\mathfrak{P}}^{4}$ such that for every $\psi \in\left(0, \psi_{0}\right)$,

$$
\left|\boldsymbol{\zeta}_{t}^{P^{\psi}}-\boldsymbol{\zeta}^{\psi}\left(t, \mathbf{X}_{t}\right)\right| \leq K_{0}\left(t, \mathbf{X}_{t}\right) \psi^{2} \mathrm{~d} t \times P^{\psi} \text {-a.e. }
$$

For each $\psi>0$, define the candidate value function $w^{\psi}: \overline{\mathbf{D}^{0}} \times \mathbb{R} \rightarrow \mathbb{R}$ by

$$
w^{\psi}(t, \mathbf{x}, y)=U(y)-U^{\prime}(y) \widetilde{w}(t, \mathbf{x}) \psi
$$

and set $w_{0}^{\psi}:=w^{\psi}\left(0, S_{0}, A_{0}, M_{0}, \Sigma_{0}, Y_{0}\right)$. Suppose for the moment that we have already proved the following two inequalities (cf. Lemmas 5.15 and 5.17):

$$
\begin{aligned}
& \inf _{P \in \mathfrak{P}} J^{\psi}\left(\boldsymbol{v}^{\star}, P\right) \geq w_{0}^{\psi}+o(\psi), \quad \text { as } \psi \downarrow 0, \\
& \sup _{\boldsymbol{v} \in \mathfrak{Y}} J^{\psi}\left(\boldsymbol{v}, P^{\psi}\right) \leq w_{0}^{\psi}+o(\psi), \quad \text { as } \psi \downarrow 0 .
\end{aligned}
$$

Denoting by $\lesssim$ "less or equal up to a term of order $o(\psi)$ ", we obtain from (5.3)-(5.4) that

$$
w_{0}^{\psi} \lesssim \inf _{P \in \mathfrak{P}} J^{\psi}\left(\boldsymbol{v}^{\star}, P\right) \lesssim \sup _{\boldsymbol{v} \in \mathfrak{Y})} \inf _{P \in \mathfrak{P}} J^{\psi}(\boldsymbol{v}, P) \lesssim \inf _{P \in \mathfrak{P}} \sup _{\boldsymbol{v} \in \mathfrak{Y}} J^{\psi}(\boldsymbol{v}, P) \lesssim \sup _{\boldsymbol{v} \in \mathfrak{Y})} J^{\psi}\left(\boldsymbol{v}, P^{\psi}\right) \lesssim w_{0}^{\psi}
$$

and

$$
w_{0}^{\psi} \lesssim \inf _{P \in \mathfrak{P}} J^{\psi}\left(\boldsymbol{v}^{\star}, P\right) \lesssim J^{\psi}\left(\boldsymbol{v}^{\star}, P^{\psi}\right) \lesssim \sup _{\boldsymbol{v} \in \mathfrak{Y}} J^{\psi}\left(\boldsymbol{v}, P^{\psi}\right) \lesssim w_{0}^{\psi}
$$

Hence, we have equality up to a term of order $o(\psi)$ everywhere. In particular, assertion (4.14) of Theorem 4.5 holds. This completes the proof of Theorem 4.5 modulo the proof of (5.3)-(5.4). The proof of these two inequalities is based on careful estimates of the HJBI equation associated to

\footnotetext{
${ }^{36}$ Recall from Remark4.3 (a) that the delta-vega hedge $\boldsymbol{v}^{\star}$ can always be included into the set of trading strategies $\mathfrak{Y}$ by making the constant $K_{\mathfrak{Y}}$ from Assumption 4.2 (a) larger if necessary. The existence of a candidate asymptotic model family is discussed in Section 4.3
} 
the SDG (2.16). Section 5.1.1 introduces the notation used in the rest of the proof as well as some preliminary results. Sections 5.1.2 5.1.3 are purely analytic and provide the required estimates of the HJBI equation. Finally, Sections [5.1.4 and 5.1.5 contain the proofs of the inequalities (5.3) and (5.4).

\subsubsection{Notation and preliminaries}

Set $\psi_{\min }=\min \left(\psi_{\nu}, \psi_{\sigma}, \psi_{\eta}, \psi_{\xi}\right), \psi_{\max }=\max \left(\psi_{\nu}, \psi_{\sigma}, \psi_{\eta}, \psi_{\xi}\right)$ (recall (2.14) $)$, and denote by $\|Q\|_{F}$ the Frobenius norm of a matrix $Q$. Recalling that the squared uncorrelated volatility $\xi^{P}$ has to be nonnegative, let $\mathbf{Z}^{0}:=\mathbb{R}^{3} \times[0, \infty)$ be the natural range for the controls $\boldsymbol{\zeta}^{P}$. A generic element of $\mathbf{Z}^{0}$ is always denoted by $\zeta=(\nu, \sigma, \eta, \xi)^{\top}$. Next, define the function $b^{\mathcal{C}}: \mathbf{D}^{0} \times \mathbf{Z}^{0} \rightarrow \mathbb{R}$ by

$$
\begin{aligned}
b^{\mathcal{C}}(t, \mathbf{x} ; \boldsymbol{\zeta}) & =\nu \mathcal{C}_{\Sigma}+\frac{1}{2} S^{2} \mathcal{C}_{S S}\left(\sigma^{2}-\Sigma^{2}\right)+\sigma \eta S \mathcal{C}_{S \Sigma}+\frac{1}{2}\left(\eta^{2}+\xi\right) \mathcal{C}_{\Sigma \Sigma} \\
& =\mathbf{c}(t, \mathbf{x})^{\top}\left(\boldsymbol{\zeta}-\boldsymbol{\zeta}^{0}(\Sigma)\right)+\frac{1}{2}\left(\begin{array}{c}
\sigma-\Sigma \\
\eta
\end{array}\right)^{\top}\left(\begin{array}{cc}
S^{2} \mathcal{C}_{S S} & S \mathcal{C}_{S \Sigma} \\
S \mathcal{C}_{S \Sigma} & \mathcal{C}_{\Sigma \Sigma}
\end{array}\right)\left(\begin{array}{c}
\sigma-\Sigma \\
\eta
\end{array}\right)
\end{aligned}
$$

cf. (4.1) for the definition of the vega-gamma-vanna-volga vector $\mathbf{c}(t, \mathbf{x})$ of the call. This definition is motivated by the drift condition (2.7), which states that $b^{\mathcal{C}}\left(t, \mathbf{X}_{t} ; \boldsymbol{\zeta}_{t}^{P}\right)=0 \mathrm{~d} t \times P$-a.e. for every $P \in \mathfrak{P}^{0}$. For each $(t, \mathbf{x}) \in \mathbf{D}$, write

$$
\mathbf{Z}^{0}(t, \mathbf{x})=\left\{\boldsymbol{\zeta} \in \mathbf{Z}^{0}: b^{\mathcal{C}}(t, \mathbf{x} ; \boldsymbol{\zeta})=0\right\}
$$

for the set of controls $\boldsymbol{\zeta}$ that fulfil the drift condition at $(t, \mathbf{x})$, and define

$$
\mathbf{Z}_{\text {lin }}^{0}(t, \mathbf{x})=\left\{\boldsymbol{\zeta} \in \mathbf{Z}^{0}: \mathbf{c}(t, \mathbf{x})^{\top}\left(\boldsymbol{\zeta}-\boldsymbol{\zeta}^{0}(\Sigma)\right)=0\right\}
$$

the set of controls $\boldsymbol{\zeta}$ that satisfy the "linearised drift condition" at $(t, \mathbf{x})$. Next, set

$$
\mathbf{Z}=[\underline{\nu}, \bar{\nu}] \times[\underline{\sigma}, \bar{\sigma}] \times[\underline{\eta}, \bar{\eta}] \times[0, \bar{\xi}]
$$

for the range of the controls in $\mathfrak{P}$ (cf. Assumption $4.2(\mathrm{~b})$ and denote by $\mathbf{Z}(t, \mathbf{x})=\mathbf{Z}^{0}(t, \mathbf{x}) \cap \mathbf{Z}$ and $\mathbf{Z}_{\text {lin }}(t, \mathbf{x})=\mathbf{Z}_{\text {lin }}^{0}(t, \mathbf{x}) \cap \mathbf{Z}$ the intersections of $\mathbf{Z}^{0}(t, \mathbf{x})$ and $\mathbf{Z}_{\text {lin }}^{0}(t, \mathbf{x})$ with $\mathbf{Z}$, respectively. Also recall from Definition 2.4 that the reference feedback control is $\zeta^{0}(\Sigma)=(0, \Sigma, 0,0)^{\top}$.

We start with the probabilistic representation of the solution to the PDE (4.7) for the cash equivalent $\widetilde{w}_{0}=\widetilde{w}\left(0, \mathbf{X}_{0}\right)$.

Proposition 5.1 (Feynman-Kac representation). Let $P^{0} \in \mathfrak{P}$ be a reference model. Then

$$
\widetilde{w}_{0}=\widetilde{w}\left(0, \mathbf{X}_{0}\right)=\frac{1}{2} E^{P^{0}}\left[\int_{0}^{T} \widetilde{g}\left(t, \mathbf{X}_{t}\right) \mathrm{d} t\right]
$$

Proof. We only sketch the standard proof. Applying Itô's formula to $\widetilde{w}\left(t, \mathbf{X}_{t}\right)$ under $P^{0}$ and using the PDE (4.7) for $\widetilde{w}$ shows that

$$
\widetilde{w}\left(0, \mathbf{X}_{0}\right)=\frac{1}{2} \int_{0}^{T} \widetilde{g}\left(t, \mathbf{X}_{t}\right) \mathrm{d} t+(\text { local martingale }) .
$$

Using Assumption 4.2 (e) the local martingale term is easily shown to be a martingale. Hence, taking expectations yields the Feynman-Kac representation (5.7).

The next lemma provides the dynamics of the P\&L processes:

Lemma 5.2. Let $\boldsymbol{v}=(\theta, \phi) \in \mathfrak{Y}$ and $P \in \mathfrak{P}$. Then under $P$,

$$
\begin{aligned}
\mathrm{d} Y_{t}^{\boldsymbol{v}, P}=( & \left.\theta_{t}-\left(\Delta\left(t, \mathbf{X}_{t}\right)-\phi_{t} \mathcal{C}_{S}\left(t, S_{t}, \Sigma_{t}\right)\right)\right) \mathrm{d} S_{t}+\left(\phi_{t} \mathcal{C}_{\Sigma}\left(t, S_{t}, \Sigma_{t}\right)-\mathcal{V}_{\Sigma}\left(t, \mathbf{X}_{t}\right)\right) \mathrm{d} \Sigma_{t}^{c, P} \\
& -b^{\mathcal{V}}\left(t, \mathbf{X}_{t} ; \boldsymbol{\zeta}_{t}^{P}\right) \mathrm{d} t .
\end{aligned}
$$


Here,

$$
\Sigma^{c, P}=\Sigma-\int_{0}^{\cdot} \nu_{u}^{P} \mathrm{~d} u
$$

is the (continuous) local martingale part of $\Sigma$ under $P$ and $b^{\mathcal{V}}: \mathbf{D}^{0} \times \mathbf{Z}^{0} \rightarrow \mathbb{R}$ is given by

$$
\begin{aligned}
b^{\mathcal{V}}(t, \mathbf{x} ; \boldsymbol{\zeta}) & =\nu \mathcal{V}_{\Sigma}+\frac{1}{2}\left(\beta \mathcal{V}_{A}+S^{2} \Gamma\right)\left(\sigma^{2}-\Sigma^{2}\right)+\sigma \eta S \frac{\partial \Delta}{\partial \Sigma}+\frac{1}{2}\left(\eta^{2}+\xi\right) \mathcal{V}_{\Sigma \Sigma} \\
& =\mathbf{v}(t, \mathbf{x})^{\top}\left(\boldsymbol{\zeta}-\boldsymbol{\zeta}^{0}(\Sigma)\right)+\frac{1}{2}\left(\begin{array}{c}
\sigma-\Sigma \\
\eta
\end{array}\right)^{\top}\left(\begin{array}{cc}
\beta \mathcal{V}_{A}+S^{2} \Gamma & S \frac{\partial \Delta}{\partial \Sigma} \\
S \frac{\partial \Delta}{\partial \Sigma} & \mathcal{V}_{\Sigma \Sigma}
\end{array}\right)\left(\begin{array}{c}
\sigma-\Sigma \\
\eta
\end{array}\right)
\end{aligned}
$$

where $\mathbf{v}$ is the vega-gamma-vanna-volga vector of the non-traded option (cf. (4.2)).

Proof. Fix $\boldsymbol{v}=(\theta, \phi) \in \mathfrak{Y}$ and $P \in \mathfrak{P}$ and recall from (2.11) and (2.10) that

$$
\mathrm{d} Y_{t}^{\boldsymbol{v}, P}=\theta_{t} \mathrm{~d} S_{t}+\phi_{t} \mathrm{~d} C_{t}-\mathrm{d} V_{t},
$$

where $V_{t}=\mathcal{V}\left(t, S_{t}, A_{t}, M_{t}, \Sigma_{t}\right)$. Thus, it remains to compute the dynamics of $C$ and $V$ under $P$.

First, by (2.4), Itô's formula (under $P$ ), and the drift condition (2.7), we have

$$
\mathrm{d} C_{t}=\mathcal{C}_{S} \mathrm{~d} S_{t}+\mathcal{C}_{\Sigma} \mathrm{d} \Sigma_{t}^{c, P}
$$

Second, applying Itô's formula to $V_{t}=\mathcal{V}\left(t, S_{t}, A_{t}, M_{t}, \Sigma_{t}\right)$ and using the PDE (2.9) to substitute $\mathcal{V}_{t}=\mathcal{V}_{t}\left(t, \mathbf{X}_{t}\right)$ and to eliminate the $\mathrm{d} M_{t}$-term, we arrive at

$$
\mathrm{d} V_{t}=\Delta \mathrm{d} S_{t}+\mathcal{V}_{\Sigma} \mathrm{d} \Sigma_{t}^{c, P}+b^{\mathcal{V}}\left(\boldsymbol{\zeta}_{t}^{P}\right) \mathrm{d} t .
$$

Finally, inserting (5.11) and (5.12) into (5.10) yields (5.8). The last equality in the definition (5.9) of $b^{\mathcal{V}}$ is the Taylor expansion of $b^{\mathcal{V}}(\boldsymbol{\zeta})$ around $\boldsymbol{\zeta}^{0}(\Sigma)$ and can be verified by computing the gradient and the Hessian of $b^{\mathcal{V}}(\boldsymbol{\zeta})$ at $\zeta^{0}(\Sigma)$.

We next analyse the dynamics of the $\mathrm{P} \& \mathrm{~L}$ process $Y^{\boldsymbol{v}^{\star}, P}$ corresponding to the delta-vega hedge $\boldsymbol{v}^{\star}$. To this end we define, for each $(t, \mathbf{x}) \in \mathbf{D}^{0}$ :

$$
\boldsymbol{v}^{\star}(t, \mathbf{x})=\left(\Delta-\frac{\mathcal{V}_{\Sigma}}{\mathcal{C}_{\Sigma}} \mathcal{C}_{S}, \frac{\mathcal{V}_{\Sigma}}{\mathcal{C}_{\Sigma}}\right)
$$

Note that, with a slight abuse of notation, we use the symbol $\boldsymbol{v}^{\star}$ both for the function defined in (5.13) and the delta-vega hedge defined in Theorem 4.5. This is, of course, motivated by the relationship $\boldsymbol{v}_{t}^{\star}=\boldsymbol{v}^{\star}\left(t, \mathbf{X}_{t}\right) 37$ The following corollary to Lemma 5.2 shows that the P\&L process $Y^{\boldsymbol{v}^{\star}, P}$ corresponding to the delta-vega hedge $\boldsymbol{v}^{\star}$ has no local martingale part and is bounded, uniformly in $P \in \mathfrak{P}$.

Corollary 5.3. There are constants $\underline{Y}, \bar{Y} \in \mathbb{R}$ such that for each $P \in \mathfrak{P}$,

$$
Y^{\boldsymbol{v}^{\star}, P} \in[\underline{Y}, \bar{Y}] \mathrm{d} t \times P \text {-a.e. }
$$

Moreover, under each $P \in \mathfrak{P}$,

$$
\mathrm{d} Y_{t}^{\boldsymbol{v}^{\star}, P}=-b^{\mathcal{V}}\left(t, \mathbf{X}_{t} ; \boldsymbol{\zeta}_{t}^{P}\right) \mathrm{d} t
$$

where $b^{\mathcal{V}}$ is defined in (5.9).

Proof. By construction of $\boldsymbol{v}_{t}^{\star}=\boldsymbol{v}^{\star}\left(t, \mathbf{X}_{t}\right)$, the local martingale part in the dynamics (5.8) of $Y^{\boldsymbol{v}^{\star}, P}$ is zero for each $P \in \mathfrak{P}$. Thus, it suffices to find a uniform bound (independent of $P \in \mathfrak{P}$ ) for the drift coefficient $b^{\mathcal{V}}\left(t, \mathbf{X}_{t} ; \boldsymbol{\zeta}_{t}^{P}\right)$. But this is immediate from Assumption 4.2 (b) and (d).

\footnotetext{
${ }^{37}$ With a slight abuse of notation, $\boldsymbol{v}_{t}^{\star}$ always denotes the time- $t$ value of the process $\boldsymbol{v}^{\star}$ and not the partial derivative of the function $\boldsymbol{v}^{\star}$ with respect to the first variable.
} 
Lemma 5.2 together with the covariations of $S$ and $\Sigma$ in (2.6) and the semimartingale decomposition (2.8) of $A$ specifies the joint dynamics of the process $\left(S, A, M, \Sigma, Y^{\boldsymbol{v}, P}\right)$ under $P \in \mathfrak{P}$. This allows to write down the Hamilton-Jacobi-Bellman-Isaacs equation corresponding to the SDG (2.16): for each $\psi>0$, the HJBI equation reads as

$$
w_{t}^{\psi}(t, \mathbf{x}, y)+\sup _{\boldsymbol{v} \in \mathbb{R}^{2}} \inf _{\boldsymbol{\zeta} \in \mathbf{Z}(t, \mathbf{x})} H^{\psi}(t, \mathbf{x}, y ; \boldsymbol{v}, \boldsymbol{\zeta})=0
$$

where the Hamiltonian $38 H^{\psi}: \mathbf{D}^{0} \times \mathbb{R} \times \mathbb{R}^{2} \times \mathbf{Z}^{0} \rightarrow \mathbb{R}$ is given by

$$
\begin{aligned}
H^{\psi}(t, \mathbf{x}, y ; \boldsymbol{v}, \boldsymbol{\zeta})=\frac{1}{\psi} & U^{\prime}(y) f(\Sigma, \boldsymbol{\zeta})+\nu w_{\Sigma}^{\psi}+\left(\alpha+\frac{1}{2} \beta \sigma^{2}\right) w_{A}^{\psi}-b^{\mathcal{V}}(\boldsymbol{\zeta}) w_{Y}^{\psi} \\
& +\frac{1}{2} \sigma^{2} S^{2}\left(w_{S S}^{\psi}+2 \gamma w_{S A}^{\psi}+\gamma^{2} w_{A A}^{\psi}\right) \\
& +\sigma S \eta\left(w_{S \Sigma}^{\psi}+\gamma w_{A \Sigma}^{\psi}\right) \\
& +\frac{1}{2}\left(\eta^{2}+\xi\right) w_{\Sigma \Sigma}^{\psi} \\
+ & \sigma^{2} S^{2}\left[\theta-\left(\Delta-\phi \mathcal{C}_{S}\right)\right]\left(w_{S Y}^{\psi}+\gamma w_{A Y}^{\psi}\right)+\sigma S \eta\left[\phi \mathcal{C}_{\Sigma}-\mathcal{V}_{\Sigma}\right]\left(w_{S Y}^{\psi}+\gamma w_{A Y}^{\psi}\right) \\
& +\sigma S \eta\left[\theta-\left(\Delta-\phi \mathcal{C}_{S}\right)\right] w_{\Sigma Y}^{\psi}+\left[\phi \mathcal{C}_{\Sigma}-\mathcal{V}_{\Sigma}\right]\left(\eta^{2}+\xi\right) w_{\Sigma Y}^{\psi} \\
& +\frac{1}{2} \sigma^{2} S^{2}\left[\theta-\left(\Delta-\phi \mathcal{C}_{S}\right)\right]^{2} w_{Y Y}^{\psi}+\frac{1}{2}\left(\eta^{2}+\xi\right)\left[\phi \mathcal{C}_{\Sigma}-\mathcal{V}_{\Sigma}\right]^{2} w_{Y Y}^{\psi} \\
& +\sigma S \eta\left[\theta-\left(\Delta-\phi \mathcal{C}_{S}\right)\right]\left[\phi \mathcal{C}_{\Sigma}-\mathcal{V}_{\Sigma}\right] w_{Y Y}^{\psi} .
\end{aligned}
$$

We emphasise that our candidate value function $w^{\psi}$ defined in (5.2) does not solve the HJBI equation (5.14) exactly. However, a key step in the proof of the two inequalities (5.3) -(5.4) is to show that $w^{\psi}$ is asymptotically (in a suitable sense) a solution to (5.14); cf. Lemmas 5.13 and 5.14 below.

We close this preliminary section by providing an auxiliary lemma that allows to estimate quantities like $b^{\mathcal{V}}(t, \mathbf{x} ; \boldsymbol{\zeta})$ or $b^{\mathcal{C}}(t, \mathbf{x} ; \boldsymbol{\zeta})$ in terms of $\left|\boldsymbol{\zeta}-\boldsymbol{\zeta}^{0}(\Sigma)\right|$.

Lemma 5.4. Define the function $q: \mathbb{R}_{+} \times \mathbb{R}^{4} \times \mathbb{R}^{4} \rightarrow \mathbb{R}$ by

$$
q(\Sigma, \mathbf{a}, \boldsymbol{\zeta})=\nu a_{1}+\frac{1}{2} a_{2}\left(\sigma^{2}-\Sigma^{2}\right)+\sigma \eta a_{3}+\frac{1}{2}\left(\eta^{2}+\xi\right) a_{4},
$$

where $\mathbf{a}=\left(a_{1}, a_{2}, a_{3}, a_{4}\right)^{\top}$ and $\boldsymbol{\zeta}=(\nu, \sigma, \eta, \xi)^{\top}$. Then:

$$
|q(\Sigma, \mathbf{a}, \boldsymbol{\zeta})| \leq \max (1, \Sigma)|\mathbf{a}|\left|\boldsymbol{\zeta}-\boldsymbol{\zeta}^{0}(\Sigma)\right|+|\mathbf{a}|\left|\boldsymbol{\zeta}-\boldsymbol{\zeta}^{0}(\Sigma)\right|^{2} .
$$

Proof. Fix $\Sigma \in \mathbb{R}_{+}, \mathbf{a} \in \mathbb{R}^{4}$, and $\zeta \in \mathbb{R}^{4}$. As $q$ is quadratic in $\boldsymbol{\zeta}$, we can recast it in matrix form:

$$
q(\Sigma, \mathbf{a}, \boldsymbol{\zeta})=\left(\begin{array}{c}
a_{1} \\
\Sigma a_{2} \\
\Sigma a_{3} \\
\frac{1}{2} a_{4}
\end{array}\right)^{\top}\left(\boldsymbol{\zeta}-\boldsymbol{\zeta}^{0}(\Sigma)\right)+\frac{1}{2}\left(\begin{array}{c}
\sigma-\Sigma \\
\eta
\end{array}\right)^{\top}\left(\begin{array}{cc}
a_{2} & a_{3} \\
a_{3} & a_{4}
\end{array}\right)\left(\begin{array}{c}
\sigma-\Sigma \\
\eta
\end{array}\right)
$$

Using the Cauchy-Schwarz inequality, the absolute value of the first summand on the right-hand side of (5.17) is easily estimated from above by $\max (1, \Sigma)|\mathbf{a}|\left|\boldsymbol{\zeta}-\boldsymbol{\zeta}^{0}(\Sigma)\right|$. Likewise, using also the compatibility of the Frobenius norm with the Euclidean norm, the absolute value of the second summand is dominated by

$$
\frac{1}{2}\left\|\left(\begin{array}{ll}
a_{2} & a_{3} \\
a_{3} & a_{4}
\end{array}\right)\right\|_{F}\left|\left(\begin{array}{c}
\sigma-\Sigma \\
\eta
\end{array}\right)\right|^{2} \leq|\mathbf{a}|\left|\boldsymbol{\zeta}-\boldsymbol{\zeta}^{0}(\Sigma)\right|^{2} .
$$

\footnotetext{
${ }^{38}$ Note that the definition of $H^{\psi}$ already contains the candidate first-order expansion $w^{\psi}$ of the value function and thus does not feature a general solution function and its derivatives as arguments.
} 


\subsubsection{Estimates for the Hamiltonian}

In order to prove that the candidate value function is - asymptotically - a solution to the HJBI equation (5.14), we need several estimates for the Hamiltonian $H^{\psi}$ defined in (5.15). To this end, we decompose it into four parts:

$$
H^{\psi}(t, \mathbf{x}, y ; \boldsymbol{v}, \boldsymbol{\zeta})=U^{\prime}(y) H_{1}^{\psi}(t, \mathbf{x} ; \boldsymbol{\zeta})+U^{\prime}(y) H_{2}^{\psi}(t, \mathbf{x}, y ; \boldsymbol{\zeta})-U^{\prime}(y) H_{3}(t, \mathbf{x} ; \boldsymbol{\zeta}) \psi-H_{4}^{\psi}(t, \mathbf{x} ; \boldsymbol{v}, \boldsymbol{\zeta})
$$

where

$$
\begin{aligned}
& H_{1}^{\psi}(t, \mathbf{x} ; \boldsymbol{\zeta}):= \frac{1}{2 \psi}\left(\boldsymbol{\zeta}-\boldsymbol{\zeta}^{0}(\Sigma)\right)^{\top} \Psi^{-1}\left(\boldsymbol{\zeta}-\boldsymbol{\zeta}^{0}(\Sigma)\right)-\mathbf{v}(t, \mathbf{x})^{\top}\left(\boldsymbol{\zeta}-\boldsymbol{\zeta}^{0}(\Sigma)\right) \\
& H_{2}^{\psi}(t, \mathbf{x}, y ; \boldsymbol{\zeta}):=-\frac{1}{2}\left(\begin{array}{c}
\sigma-\Sigma \\
\eta
\end{array}\right)^{\top}\left(\begin{array}{cc}
\beta \mathcal{V}_{A}+S^{2} \Gamma & S \frac{\partial \Delta}{\partial \Sigma} \\
S \frac{\partial \Delta}{\partial \Sigma} & \mathcal{V}_{\Sigma \Sigma}
\end{array}\right)\left(\begin{array}{c}
\sigma-\Sigma \\
\eta
\end{array}\right)+\frac{U^{\prime \prime}(y)}{U^{\prime}(y)} b^{\mathcal{V}}(\boldsymbol{\zeta}) \widetilde{w} \psi \\
& H_{3}(t, \mathbf{x} ; \boldsymbol{\zeta}):=\nu \widetilde{w}_{\Sigma}+\left(\alpha+\frac{1}{2} \beta \sigma^{2}\right) \widetilde{w}_{A}+\frac{1}{2} \sigma^{2} S^{2}\left(\widetilde{w}_{S S}+2 \gamma \widetilde{w}_{S A}+\gamma^{2} \widetilde{w}_{A A}\right) \\
&+ \sigma \eta S\left(\widetilde{w}_{S \Sigma}+\gamma \widetilde{w}_{A \Sigma}\right)+\frac{1}{2}\left(\eta^{2}+\xi\right) \widetilde{w}_{\Sigma \Sigma}, \\
& H_{4}^{\psi}(t, \mathbf{x}, y ; \boldsymbol{v}, \boldsymbol{\zeta}):=- \frac{w_{Y Y}^{\psi}}{2}\left(\begin{array}{c}
\sigma S\left(\theta-\left(\Delta-\phi \mathcal{C}_{S}\right)\right) \\
\phi \mathcal{C}_{\Sigma}-\mathcal{V}_{\Sigma}
\end{array}\right)^{\top}\left(\begin{array}{cc}
1 & \eta \\
\eta & \eta^{2}+\xi
\end{array}\right)\left(\begin{array}{c}
\sigma S\left(\theta-\left(\Delta-\phi \mathcal{C}_{S}\right)\right) \\
\phi \mathcal{C}_{\Sigma}-\mathcal{V}_{\Sigma}
\end{array}\right) \\
&+\psi U^{\prime \prime}(y)\left(\begin{array}{c}
\sigma S\left(\widetilde{w}_{S}+\gamma \widetilde{w}_{A}\right) \\
\widetilde{w}_{\Sigma}
\end{array}\right)^{\top}\left(\begin{array}{cc}
1 & \eta \\
\eta & \eta^{2}+\xi
\end{array}\right)\left(\begin{array}{c}
\sigma S\left(\theta-\left(\Delta-\phi \mathcal{C}_{S}\right)\right) \\
\phi \mathcal{C}_{\Sigma}-\mathcal{V}_{\Sigma}
\end{array}\right) .
\end{aligned}
$$

$H_{1}^{\psi}$ includes the penalty term (cf. the definition of $f$ in (2.13) $)$ and the linear $O(1)$ part of $b^{\mathcal{V}}(\boldsymbol{\zeta}) w_{Y}^{\psi}$; $H_{2}^{\psi}$ contains the quadratic $O(1)$ part and the $O(\psi)$ part of $b^{\mathcal{V}}(\boldsymbol{\zeta}) w_{Y}^{\psi} ; H_{4}^{\psi}$ collects all second-order partial derivatives of $w^{\psi}$ that involve at least one partial derivative with respect to $Y$; and $H_{3}$ takes care of all remaining partial derivatives of $w^{\psi}$.

For later reference, we note that by the definition of $H_{3}$ and $\zeta^{0}$, the PDE (4.7) for $\widetilde{w}$ can be written as

$$
\widetilde{w}_{t}(t, \mathbf{x})+H_{3}\left(t, \mathbf{x} ; \boldsymbol{\zeta}^{0}(\Sigma)\right)+\frac{1}{2} \widetilde{g}(t, \mathbf{x})=0 \quad \text { for }(t, \mathbf{x}) \in \mathbf{D}
$$

Moreover, for every $(t, \mathbf{x}, y) \in \mathbf{D} \times \mathbb{R}$ and $\boldsymbol{\zeta} \in \mathbf{Z}$,

$$
H_{1}^{\psi}\left(t, \mathbf{x} ; \boldsymbol{\zeta}^{0}(\Sigma)\right)=0, \quad H_{2}^{\psi}\left(t, \mathbf{x} ; \boldsymbol{\zeta}^{0}(\Sigma)\right)=0, \quad H_{4}^{\psi}\left(t, \mathbf{x}, y ; \boldsymbol{v}^{\star}(t, \mathbf{x}), \boldsymbol{\zeta}\right)=0,
$$

by construction of the reference feedback control $\zeta^{0}$ (cf. Definition 2.4) and the delta-vega hedge $\boldsymbol{v}^{\star}(\mathrm{cf}$. (5.13) $)$.

Remark 5.5. Recall that the HJBI equation (5.14) involves a minimisation over $\boldsymbol{\zeta} \in \mathbf{Z}(t, \mathbf{x})$ and a maximisation over $\boldsymbol{v} \in \mathbb{R}^{2}$. The strategy variable $\boldsymbol{v}$ only shows up in the $H_{4}^{\psi}$ term. Moreover, using an ansatz of the form $\boldsymbol{\zeta}=\boldsymbol{\zeta}^{0}(\Sigma)+\widetilde{\boldsymbol{\zeta}} \psi$, one can check that $\widetilde{\boldsymbol{\zeta}}$ only affects the $O(\psi)$ term of $H^{\psi}$ through $H_{1}^{\psi}$ (provided that $\boldsymbol{v}=\boldsymbol{v}^{\star}$ so that the $H_{4}^{\psi}$ term vanishes; cf. (5.21)). The impact of $\widetilde{\boldsymbol{\zeta}}$ through $H_{2}^{\psi}$ and $H_{3}$ only appears at higher orders. This distinction is reflected in the proofs of this section as follows.

On the one hand, the estimates for the terms $H_{2}^{\psi}$ and $H_{3}$ in Propositions 5.9 5.10 and Corollary 5.11 are rather direct and provide simultaneously asymptotic upper and lower bounds. On the other hand, the proofs of the estimates for $H_{4}^{\psi}$ and, in particular, $H_{1}^{\psi}$ are more difficult as the corresponding bounds arise from optimisation problems over the strategy variables and the controls, respectively. The asymptotic bound for $H_{4}^{\psi}$ is the easier one because $H_{4}^{\psi}$ is quadratic in $\boldsymbol{v}$ and the optimisation is unconstrained. In contrast, the asymptotic bound for $H_{1}^{\psi}$ in Proposition 5.8 arises from the linearly constrained quadratic programming problem (3.10). An additional difficulty stems from the fact that we need this bound to hold for controls $\zeta \in \mathbf{Z}(t, \mathbf{x})$ that satisfy the nonlinear contraint $b^{\mathcal{C}}(t, \mathbf{x} ; \boldsymbol{\zeta})=0$ instead of the linear one (cf. Proposition $5.8(\mathrm{a})$ ). 
We first provide the solution to a linearly constrained quadratic programming problem involving the vega-gamma-vanna-volga vectors $\mathbf{c}(t, \mathbf{x})$ and $\mathbf{v}(t, \mathbf{x})$ that lies at the core of the minimisation part of the HJBI equation. In particular, the candidate feedback control $\boldsymbol{\zeta}^{\psi}$ (cf. (4.6) ) is a suitably modified version of the minimiser $\boldsymbol{\zeta}^{\psi *}$ (cf. (5.24) below) of this quadratic programming problem; both controls differ only by the indicator $\mathbf{1}_{\{\underline{\Sigma}<\Sigma<\bar{\Sigma}\}}$ that ensures that $\boldsymbol{\zeta}^{\psi}$ falls back to the reference feedback control $\zeta^{0}(\Sigma)$ once the implied volatility hits the boundary of $[\underline{\Sigma}, \bar{\Sigma}]$. Recall the definitions of $\Psi, \mathbf{c}, \mathbf{v}, \lambda, \mu, \widetilde{\boldsymbol{\zeta}}$, and $\boldsymbol{\zeta}^{\psi}$ in (2.14) and (4.1)-(4.6).

Lemma 5.6. For each $(t, \mathbf{x}) \in \mathbf{D}$ and $\psi>0$, consider the linearly constrained minimisation problem

$$
\text { minimise } H_{1}^{\psi}(t, \mathbf{x} ; \boldsymbol{\zeta}) \quad \text { subject to } \boldsymbol{\zeta} \in \mathbf{Z}_{\mathrm{lin}}^{0}(t, \mathbf{x}) \text {. }
$$

(a) For each $(t, \mathbf{x}) \in \mathbf{D}$ and $\psi>0$, we have

$$
\min _{\boldsymbol{\zeta} \in \mathbf{Z}_{\operatorname{lin}}^{0}(t, \mathbf{x})} H_{1}^{\psi}(t, \mathbf{x} ; \boldsymbol{\zeta})=-\frac{1}{2} \widetilde{g}(t, \mathbf{x}) \psi
$$

and the minimum is attained at

$$
\boldsymbol{\zeta}^{\psi *}(t, \mathbf{x})=\boldsymbol{\zeta}^{0}(\Sigma)+\psi \widetilde{\boldsymbol{\zeta}}(t, \mathbf{x})
$$

In particular, as $\boldsymbol{\zeta}^{\psi *} \in \mathbf{Z}_{\mathrm{lin}}^{0}(t, \mathbf{x}), \mathbf{c}(t, \mathbf{x})^{\top} \widetilde{\boldsymbol{\zeta}}(t, \mathbf{x})=0$ and $\overrightarrow{\mathbf{e}}_{4}^{\top} \widetilde{\boldsymbol{\zeta}}(t, \mathbf{x}) \geq 0$.

(b) For each $(t, \mathbf{x}) \in \mathbf{D}$ and $\psi>0,(\lambda(t, \mathbf{x}), \mu(t, \mathbf{x}))$ is a Lagrange multiplier for (5.22) (independent of $\psi$ ), i.e.,

$$
-\frac{1}{2} \widetilde{g}(t, \mathbf{x}) \psi=\inf _{\boldsymbol{\zeta} \in \mathbb{R}^{4}} L_{1}^{\psi}(t, \mathbf{x} ; \boldsymbol{\zeta}, \lambda(t, \mathbf{x}), \mu(t, \mathbf{x}))
$$

where

$$
L_{1}^{\psi}\left(t, \mathbf{x} ; \boldsymbol{\zeta}, \lambda^{\prime}, \mu^{\prime}\right)=H_{1}^{\psi}(t, \mathbf{x} ; \boldsymbol{\zeta})+\lambda^{\prime} \mathbf{c}(t, \mathbf{x})^{\top}\left(\boldsymbol{\zeta}-\boldsymbol{\zeta}^{0}(\Sigma)\right)-\mu^{\prime} \overrightarrow{\mathbf{e}}_{4}^{\top}\left(\boldsymbol{\zeta}-\boldsymbol{\zeta}^{0}(\Sigma)\right)
$$

is the Lagrangian corresponding to the constrained minimisation problem (5.22).

(c) There is $K_{\widetilde{g}}>0$ such that $0 \leq \widetilde{g} \leq K_{\widetilde{g}}$ on $\mathbf{D}$.

(d) There is $K_{\zeta} \geq 1$ such that for every $(t, \mathbf{x}) \in \mathbf{D}$ and $\psi>0$,

$$
\left|\boldsymbol{\zeta}^{\psi}(t, \mathbf{x})-\boldsymbol{\zeta}^{0}(\Sigma)\right| \leq K_{\zeta} \psi
$$

(e) For every $(t, \mathbf{x}) \in \mathbf{D}$ and $\psi>0$,

$$
H_{1}^{\psi}\left(t, \mathbf{x} ; \zeta^{\psi}(t, \mathbf{x})\right)=-\frac{1}{2} \widetilde{g}(t, \mathbf{x}) \mathbf{1}_{\{\Sigma \in(\underline{\Sigma}, \bar{\Sigma})\}} \psi .
$$

(f) There is $K_{\lambda} \in L_{\mathfrak{P}}^{2}$ such that for every $(t, \mathbf{x}) \in \mathbf{D}$,

$$
|\lambda(t, \mathbf{x})|\left\|\left(\begin{array}{cc}
S^{2} \mathcal{C}_{S S} & S \mathcal{C}_{S \Sigma} \\
S \mathcal{C}_{S \Sigma} & \mathcal{C}_{\Sigma \Sigma}
\end{array}\right)\right\|_{F} \leq K_{\lambda}(t, \mathbf{x})
$$

Proof. Recalling the definitions of $H_{1}^{\psi}(t, \mathbf{x} ; \boldsymbol{\zeta})$ (cf. (5.19) $)$ and $\mathbf{Z}_{\mathrm{lin}}^{0}(t, \mathbf{x})$ (cf. (5.6)) and using the substitution $\mathbf{z}=\boldsymbol{\zeta}-\boldsymbol{\zeta}^{0}(\Sigma)$, it is easy to see that for each $(t, \mathbf{x}) \in \mathbf{D}$ and $\psi>0$, the minimisation problem (5.22) can be recast as

$$
\text { minimise } \frac{1}{2 \psi} \mathbf{z}^{\top} \Psi^{-1} \mathbf{z}-\mathbf{v}(t, \mathbf{x})^{\top} \mathbf{z} \quad \text { subject to } \mathbf{z} \in \mathbb{R}^{4}, \mathbf{z}^{\top} \mathbf{c}(t, \mathbf{x})=0, z_{4} \geq 0 .
$$


Note that (5.27) is a linearly constrained minimisation problem of the form (A.5) with $n=4$, $D=\Psi^{-1} / \psi, \mathbf{v}=\mathbf{v}(t, \mathbf{x})$, and $\mathbf{c}=\mathbf{c}(t, \mathbf{x})$. Also note that with this choice of $D$, we have (denoting by $d_{\max }$ and $d_{\min }$ the maximal and minimal element on the diagonal of $D$, respectively) $d_{\max }=\psi_{\min }^{-1} / \psi$ and $d_{\min }=\psi_{\max }^{-1} / \psi$, so that, in particular, $\frac{d_{\max }}{d_{\min }}=\frac{\psi_{\max }}{\psi_{\min }}$

(a): Fix $(t, \mathbf{x}) \in \mathbf{D}$ and $\psi>0$. By Lemma A.1 (a), the minimiser of (5.27) is $\mathbf{z}^{*}=\psi \widetilde{\boldsymbol{\zeta}}(t, \mathbf{x})$. After resubstitution, this yields the minimiser (5.24) of the original minimisation problem (5.22). Moreover, by Lemma A.1 (c), the minimum of (5.27) (which clearly coincides with the minimum of $(5.22)$ ) is

$$
-\frac{1}{2} \mathbf{v}(t, \mathbf{x})^{\top} \mathbf{z}^{*}=-\frac{1}{2} \mathbf{v}(t, \mathbf{x})^{\top} \widetilde{\boldsymbol{\zeta}}(t, \mathbf{x}) \psi=-\frac{1}{2} \widetilde{g}(t, \mathbf{x}) \psi ;
$$

recall the definition of $\widetilde{g}$ in (4.8). For further reference, we also note that the bound on $\left|\mathbf{z}^{*}\right|$ from Lemma A.1 (a) translates to

$$
|\widetilde{\boldsymbol{\zeta}}(t, \mathbf{x})| \leq \psi_{\max }|\mathbf{v}(t, \mathbf{x})|
$$

(b): This follows immediately from the second assertion of Lemma A.1 (d).

(c): By Assumption 4.2 (d) and the definition of $\mathbf{v}(t, \mathbf{x})$, there is a constant $K_{\mathbf{v}}>0$ such that $|\mathbf{v}(t, \mathbf{x})| \leq K_{\mathbf{v}}$ for all $(t, \mathbf{x}) \in \mathbf{D}$. Set $K_{\widetilde{g}}=\psi_{\max } K_{\mathbf{v}}^{2}$ and fix $(t, \mathbf{x}) \in \mathbf{D}$ and $\psi>0$. As $\boldsymbol{\zeta}^{0}(\Sigma) \in \mathbf{Z}_{\text {lin }}^{0}(t, \mathbf{x})$ and $H_{1}^{\psi}\left(t, \mathbf{x} ; \boldsymbol{\zeta}^{0}(\Sigma)\right)=0$, we have $\widetilde{g}(t, \mathbf{x}) \geq 0$ by (5.23). On the other hand, using the Cauchy-Schwarz inequality and (5.28), we have for every $(t, \mathbf{x}) \in \mathbf{D}$ :

$$
g(t, \mathbf{x})=\mathbf{v}(t, \mathbf{x})^{\top} \widetilde{\boldsymbol{\zeta}}(t, \mathbf{x}) \leq|\mathbf{v}(t, \mathbf{x})||\widetilde{\boldsymbol{\zeta}}(t, \mathbf{x})| \leq \psi_{\max }|\mathbf{v}(t, \mathbf{x})|^{2} \leq K_{\widetilde{g}}
$$

(d): Set $K_{\boldsymbol{\zeta}}=\max \left(\psi_{\max } K_{\mathbf{v}}, 1\right)$ where $K_{\mathbf{v}}$ is as in the proof of part (c), and fix $(t, \mathbf{x}) \in \mathbf{D}$ as well as $\psi>0$. If $\Sigma \in\{\underline{\Sigma}, \bar{\Sigma}\}$, then $\zeta^{\psi}(t, \mathbf{x})=\zeta^{0}(\Sigma)$ by construction and the assertion is trivial. Otherwise, if $\Sigma \in(\underline{\Sigma}, \bar{\Sigma})$, then $\boldsymbol{\zeta}^{\psi}(t, \mathbf{x})=\boldsymbol{\zeta}^{\psi *}(t, \mathbf{x})$ and (5.28) implies that

$$
\left|\boldsymbol{\zeta}^{\psi}(t, \mathbf{x})-\boldsymbol{\zeta}^{0}(\Sigma)\right|=\left|\boldsymbol{\zeta}^{\psi *}(t, \mathbf{x})-\boldsymbol{\zeta}^{0}(\Sigma)\right|=|\widetilde{\boldsymbol{\zeta}}(t, \mathbf{x})| \psi \leq \psi_{\max }|\mathbf{v}(t, \mathbf{x})| \psi \leq K_{\boldsymbol{\zeta}} \psi
$$

(e): Fix $(t, \mathbf{x}) \in \mathbf{D}$ and $\psi>0$. First, suppose that $\Sigma \in\{\underline{\Sigma}, \bar{\Sigma}\}$. Then $\boldsymbol{\zeta}^{\psi}(t, \mathbf{x})=\zeta^{0}(\Sigma)$ by construction of $\boldsymbol{\zeta}^{\psi}$ and the assertion follows from the fact that $H_{1}^{\psi}\left(t, \mathbf{x} ; \boldsymbol{\zeta}^{0}(\Sigma)\right)=0$ (cf. (5.21)). Second, suppose that $\Sigma \in(\underline{\Sigma}, \bar{\Sigma})$. Then $\zeta^{\psi}(t, \mathbf{x})=\zeta^{\psi *}(t, \mathbf{x})$ and the assertion follows from part (a).

(f): Let $K_{\mathbf{v}}>0$ be as in the proof of part (c). Then the bound A.7) from Lemma A.1 (b) implies that, for every $(t, \mathbf{x}) \in \mathbf{D}$,

$$
\left|\lambda(t, \mathbf{x}) \mathbf{c}(t, \mathbf{x})-\mu(t, \mathbf{x}) \overrightarrow{\mathbf{e}}_{4}\right| \leq\left(1+\frac{\psi_{\max }}{\psi_{\min }}\right) K_{\mathbf{v}}
$$

Recall from (4.1) that $\mathbf{c}(t, \mathbf{x})=\left(\mathcal{C}_{\Sigma}, \Sigma S^{2} \mathcal{C}_{S S}, \Sigma S \mathcal{C}_{S \Sigma}, \frac{1}{2} \mathcal{C}_{\Sigma \Sigma}\right)^{\top}$. Clearly, each of the first three components of $\lambda(t, \mathbf{x}) \mathbf{c}(t, \mathbf{x})$ is bounded in absolute value by the length of the vector $\lambda(t, \mathbf{x}) \mathbf{c}(t, \mathbf{x})-$ $\mu(t, \mathbf{x}) \overrightarrow{\mathbf{e}}_{4}$. Using also that $\underline{\Sigma}>0$, we can find a constant $K>0$ such that for every $(t, \mathbf{x})=$ $(t, S, A, M, \Sigma) \in \mathbf{D}$

$$
\left|\lambda(t, \mathbf{x}) \mathcal{C}_{\Sigma}(t, S, \Sigma)\right| \leq K, \quad\left|\lambda(t, \mathbf{x}) S^{2} \mathcal{C}_{S S}(t, S, \Sigma)\right| \leq K, \quad\left|\lambda(t, \mathbf{x}) S \mathcal{C}_{S \Sigma}(t, S, \Sigma)\right| \leq K .
$$

(This argument does not work for the fourth component of $\lambda(t, \mathbf{x}) \mathbf{c}(t, \mathbf{x})$ due to the presence of the term $\mu(t, \mathbf{x}) \overrightarrow{\mathbf{e}}_{4}$ in (5.30).) Set $K_{\lambda}(t, \mathbf{x})=3 K\left(2+K_{\mathcal{C}}(t, \mathbf{x})\right)$ where $K_{\mathcal{C}} \in L_{\mathfrak{P}}^{2}$ is as in Assumption $4.2(\mathrm{c})$. Clearly, $K_{\lambda} \in L_{\mathfrak{P}}^{2}$.

Now, fix $(t, \mathbf{x}) \in \mathbf{D}$. Using that the Euclidean norm of a vector is dominated by the sum of the absolute values of each of its entries as well as Assumption 4.2 (c) to bound $\left|\mathcal{C}_{\Sigma \Sigma}\right|$,

$$
\begin{aligned}
|\lambda(t, \mathbf{x})|\left\|\left(\begin{array}{cc}
S^{2} \mathcal{C}_{S S} & S \mathcal{C}_{S \Sigma} \\
S \mathcal{C}_{S \Sigma} & \mathcal{C}_{\Sigma \Sigma}
\end{array}\right)\right\|_{F} & =|\lambda(t, \mathbf{x})|\left|\left(S^{2} C_{S S}, S \mathcal{C}_{S \Sigma}, S \mathcal{C}_{S \Sigma}, \mathcal{C}_{\Sigma \Sigma}\right)\right| \\
& \leq|\lambda(t, \mathbf{x})|\left(\left|S^{2} \mathcal{C}_{S S}\right|+2\left|S \mathcal{C}_{S \Sigma}\right|+\left|\mathcal{C}_{\Sigma \Sigma}\right|\right) \\
& \leq\left(2+K_{\mathcal{C}}(t, \mathbf{x})\right)|\lambda(t, \mathbf{x})|\left(\left|\mathcal{C}_{\Sigma}\right|+\left|S^{2} \mathcal{C}_{S S}\right|+\left|S \mathcal{C}_{S \Sigma}\right|\right)
\end{aligned}
$$

Combining this with (5.31) and the choice of $K_{\lambda}$ completes the proof. 
Corollary 5.7. For each $(t, \mathbf{x}) \in \mathbf{D}$,

$$
\widetilde{g}(t, \mathbf{x})=-\Sigma\left(\phi^{\star} S^{2} \mathcal{C}_{S S}-\left(\beta \mathcal{V}_{A}+S^{2} \Gamma\right)\right) \widetilde{\sigma}-\Sigma\left(\phi^{\star} S \mathcal{C}_{S \Sigma}-S \frac{\partial \Delta}{\partial \Sigma}\right) \widetilde{\eta}-\frac{1}{2}\left(\phi^{\star} \mathcal{C}_{\Sigma \Sigma}-\mathcal{V}_{\Sigma \Sigma}\right) \widetilde{\xi},
$$

where the functions $(\widetilde{\nu}, \widetilde{\sigma}, \widetilde{\eta}, \widetilde{\xi})=\widetilde{\zeta}$ are defined in (4.5) and $\phi^{\star}=\frac{\mathcal{V}_{\Sigma}}{\mathcal{C}_{\Sigma}}$.

Proof. We fix $(t, \mathbf{x}) \in \mathbf{D}$ and drop all arguments in the following to ease the notation. Recall that $\widetilde{g}=\mathbf{v}^{\top} \widetilde{\boldsymbol{\zeta}}$ by definition (cf. (4.8) ). Moreover, $\mathbf{c}^{\top} \widetilde{\boldsymbol{\zeta}}=0$ by Lemma 5.6 (a). Hence,

$$
\widetilde{g}=\left(\mathbf{v}-\phi^{\star} \mathbf{c}\right)^{\top} \widetilde{\boldsymbol{\zeta}}
$$

Note that the first component of $\mathbf{v}-\phi^{\star} \mathbf{c}$ is zero by the choice of $\phi^{\star}$ (the vega hedge neutralises the portfolio vega). Now the assertion follows from the definitions of $\mathbf{c}$ and $\mathbf{v}$ in (4.1) and (4.2).

The remainder of this subsection provides estimates for the four terms $H_{1}^{\psi}, H_{2}^{\psi}, H_{3}$, and $H_{4}^{\psi}$. Roughly speaking, part (a) of the first of the following propositions shows that $-\frac{1}{2} \widetilde{g}(t, \mathbf{x}) \psi$ is not only a lower bound for $H_{1}^{\psi}(t, \mathbf{x} ; \boldsymbol{\zeta})$ over $\boldsymbol{\zeta} \in \mathbf{Z}_{\text {lin }}^{0}(t, \mathbf{x})$ (as is shown by Lemma 5.6), but also, up to a term of order $O\left(\psi^{2}\right)$, for $\boldsymbol{\zeta} \in \mathbf{Z}(t, \mathbf{x})$ that are close to $\zeta^{0}(\Sigma)$. Moreover, part (b) shows that this lower bound is approximately attained by controls $\zeta$ that are close to the candidate feedback control $\boldsymbol{\zeta}^{\psi}$.

Proposition 5.8 ( $H_{1}^{\psi}$ estimate).

(a) Let $0 \leq K \in L_{\mathfrak{P}}^{4}$. There is a nonnegative $K_{1} \in L_{\mathfrak{P}}^{1}$ (depending on $K$ ) such that for every $(t, \mathbf{x}) \in \mathbf{D}, \boldsymbol{\zeta} \in \mathbf{Z}(t, \mathbf{x})$, and $\psi \in(0,1)$ satisfying

$$
\left|\boldsymbol{\zeta}-\boldsymbol{\zeta}^{0}(\Sigma)\right| \leq K(t, \mathbf{x}) \psi
$$

we have

$$
H_{1}^{\psi}(t, \mathbf{x} ; \boldsymbol{\zeta}) \geq-\frac{1}{2} \widetilde{g}(t, \mathbf{x}) \psi-K_{1}(t, \mathbf{x}) \psi^{2}
$$

(b) Let $0 \leq \bar{K} \in L_{\mathfrak{P}}^{4}$. There is a nonnegative $K_{1} \in L_{\mathfrak{P}}^{2}$ (depending on $\bar{K}$ ) such that for every $(t, \mathbf{x}) \in \mathbf{D}, \boldsymbol{\zeta} \in \mathbf{Z}$, and $\psi \in(0,1)$ satisfying

$$
\left|\boldsymbol{\zeta}-\boldsymbol{\zeta}^{\psi}(t, \mathbf{x})\right| \leq \bar{K}(t, \mathbf{x}) \psi^{2},
$$

we have

$$
H_{1}^{\psi}(t, \mathbf{x} ; \boldsymbol{\zeta}) \leq-\frac{1}{2} \widetilde{g}(t, \mathbf{x}) \mathbf{1}_{\{\Sigma \in(\underline{\Sigma}, \bar{\Sigma})\}} \psi+K_{1}(t, \mathbf{x}) \psi^{2} .
$$

Proof. (a): Choose $0 \leq K_{\lambda} \in L_{\mathfrak{P}}^{2}$ as in Lemma 5.6 (f) and set $K_{1}(t, \mathbf{x})=\frac{1}{2} K_{\lambda}(t, \mathbf{x}) K(t, \mathbf{x})^{2}$. As $K \in L_{\mathfrak{P}}^{4}$ and $K_{\lambda} \in L_{\mathfrak{P}}^{2}$, it follows that $K_{1} \in L_{\mathfrak{P}}^{1}$. Now, fix $(t, \mathbf{x}) \in \mathbf{D}, \boldsymbol{\zeta} \in \mathbf{Z}(t, \mathbf{x})$, and $\psi \in(0,1)$ satisfying (5.32). As $\boldsymbol{\zeta}=(\nu, \sigma, \eta, \xi) \in \mathbf{Z}(t, \mathbf{x})$, we have $b^{\mathcal{C}}(t, \mathbf{x} ; \boldsymbol{\zeta})=0$ and $\xi \geq 0$. Hence, using also that $\mu(t, \mathbf{x}) \geq 0$ by definition (cf. (4.4)),

$$
H_{1}^{\psi}(t, \mathbf{x} ; \boldsymbol{\zeta}) \geq H_{1}^{\psi}(t, \mathbf{x} ; \boldsymbol{\zeta})+\lambda(t, \mathbf{x}) b^{\mathcal{C}}(t, \mathbf{x} ; \boldsymbol{\zeta})-\mu(t, \mathbf{x}) \xi .
$$

Substituting the expression (5.5) for $b^{\mathcal{C}}$ and using the definition (5.25) of the Lagrangian $L_{1}^{\psi}$, we obtain

$$
H_{1}^{\psi}(t, \mathbf{x} ; \boldsymbol{\zeta}) \geq L_{1}^{\psi}(t, \mathbf{x} ; \boldsymbol{\zeta}, \lambda(t, \mathbf{x}), \mu(t, \mathbf{x}))+\frac{\lambda(t, \mathbf{x})}{2}\left(\begin{array}{c}
\sigma-\Sigma \\
\eta
\end{array}\right)^{\top}\left(\begin{array}{cc}
S^{2} \mathcal{C}_{S S} & S \mathcal{C}_{S \Sigma} \\
S \mathcal{C}_{S \Sigma} & \mathcal{C}_{\Sigma \Sigma}
\end{array}\right)\left(\begin{array}{c}
\sigma-\Sigma \\
\eta
\end{array}\right)
$$


The first term on the right-hand side is bounded from below by $-\frac{1}{2} \widetilde{g}(t, \mathbf{x}) \psi$ by Lemma 5.6 (b). To estimate the second term, we use the Cauchy-Schwarz inequality, the compatibility of the Frobenius norm with the Euclidean norm, and the fact that $(\sigma-\Sigma, \eta)$ is just the second and third component of $\boldsymbol{\zeta}-\zeta^{0}(\Sigma)$. As a result, we find that

$$
H_{1}^{\psi}(t, \mathbf{x} ; \boldsymbol{\zeta}) \geq-\frac{1}{2} \widetilde{g}(t, \mathbf{x}) \psi-\frac{1}{2}|\lambda(t, \mathbf{x})|\left\|\left(\begin{array}{cc}
S^{2} \mathcal{C}_{S S} & S \mathcal{C}_{S \Sigma} \\
S \mathcal{C}_{S \Sigma} & \mathcal{C}_{\Sigma \Sigma}
\end{array}\right)\right\|_{F}\left|\boldsymbol{\zeta}-\boldsymbol{\zeta}^{0}(\Sigma)\right|^{2}
$$

Finally, condition (5.32) and the bound (5.26) from Lemma 5.6 (f) give

$$
H_{1}^{\psi}(t, \mathbf{x} ; \boldsymbol{\zeta}) \geq-\frac{1}{2} \widetilde{g}(t, \mathbf{x}) \psi-\frac{1}{2} K_{\lambda}(t, \mathbf{x}) K(t, \mathbf{x})^{2} \psi^{2} .
$$

This proves assertion (a) by the choice of $K_{1}$.

(b): Set $K^{\prime}(t, \mathbf{x})=K_{\zeta}+\bar{K}(t, \mathbf{x})$ where $K_{\zeta}$ is chosen as in Lemma 5.6 (d). Clearly, $K^{\prime} \in L_{\mathfrak{P}}^{4}$. By Assumption 4.2 (d) and the definition of $\mathbf{v}(t, \mathbf{x})$, there is a constant $K_{\mathbf{v}}>0$ such that $|\mathbf{v}(t, \mathbf{x})| \leq K_{\mathbf{v}}$ for all $(t, \mathbf{x}) \in \mathbf{D}$. Next, set $K_{1}(t, \mathbf{x})=\left(\psi_{\min }^{-1} K^{\prime}(t, \mathbf{x})+K_{\mathbf{v}}\right) \bar{K}(t, \mathbf{x})$. Since $K^{\prime}, \bar{K} \in L_{\mathfrak{P}}^{4}$, it follows that $K_{1} \in L_{\mathfrak{P}}^{2}$.

Now, fix $(t, \mathbf{x}) \in \mathbf{D}, \boldsymbol{\zeta} \in \mathbf{Z}$, and $\psi \in(0,1)$ satisfying (5.33). For brevity, we write $\boldsymbol{\zeta}^{\psi}=\boldsymbol{\zeta}^{\psi}(t, \mathbf{x})$ and $\boldsymbol{\zeta}^{0}=\boldsymbol{\zeta}^{0}(\Sigma)$. Now, by the multivariate mean-value theorem, there is $\ell \in[0,1]$ such that

$$
H_{1}^{\psi}(t, \mathbf{x} ; \boldsymbol{\zeta})=H_{1}^{\psi}\left(t, \mathbf{x} ; \boldsymbol{\zeta}^{\psi}\right)+\mathrm{D}_{\boldsymbol{\zeta}} H_{1}^{\psi}\left(t, \mathbf{x} ; \boldsymbol{\zeta}_{\ell}\right)^{\top}\left(\boldsymbol{\zeta}-\boldsymbol{\zeta}^{\psi}\right)
$$

where $\boldsymbol{\zeta}_{\ell}=(1-\ell) \boldsymbol{\zeta}^{\psi}+\ell \boldsymbol{\zeta}$. By the definition of $H_{1}^{\psi}$, we have

$$
\mathrm{D}_{\zeta} H_{1}^{\psi}\left(t, \mathbf{x} ; \boldsymbol{\zeta}_{\ell}\right)=\frac{1}{\psi} \Psi^{-1}\left(\boldsymbol{\zeta}_{\ell}-\boldsymbol{\zeta}^{0}\right)-\mathbf{v}(t, \mathbf{x}) .
$$

By Lemma 5.6 (d) and (5.33),

$$
\begin{aligned}
\left|\boldsymbol{\zeta}_{\ell}-\boldsymbol{\zeta}^{0}\right| & =\left|\boldsymbol{\zeta}^{\psi}-\boldsymbol{\zeta}^{0}+\ell\left(\boldsymbol{\zeta}-\boldsymbol{\zeta}^{\psi}\right)\right| \leq\left|\boldsymbol{\zeta}^{\psi}-\boldsymbol{\zeta}^{0}\right|+\ell\left|\boldsymbol{\zeta}-\boldsymbol{\zeta}^{\psi}\right| \\
& \leq K_{\boldsymbol{\zeta}} \psi+\bar{K}(t, \mathbf{x}) \psi^{2} \leq K^{\prime}(t, \mathbf{x}) \psi
\end{aligned}
$$

so that

$$
\left|\mathrm{D}_{\zeta} H_{1}^{\psi}\left(t, \mathbf{x} ; \boldsymbol{\zeta}_{\ell}\right)\right| \leq \psi_{\min }^{-1} K^{\prime}(t, \mathbf{x})+K_{\mathbf{v}} .
$$

Moreover, by Lemma 5.6 (e),

$$
H_{1}^{\psi}\left(t, \mathbf{x} ; \boldsymbol{\zeta}^{\psi}\right)=-\frac{1}{2} \widetilde{g}(t, \mathbf{x}) \mathbf{1}_{\{\Sigma \in(\underline{\Sigma}, \bar{\Sigma})\}} \psi .
$$

Combining this with (5.35) and (5.33) in (5.34), we obtain

$$
H_{1}^{\psi}(t, \mathbf{x} ; \boldsymbol{\zeta}) \leq-\frac{1}{2} \widetilde{g}(t, \mathbf{x}) \mathbf{1}_{\{\Sigma \in(\underline{\Sigma}, \bar{\Sigma})\}} \psi+\left(\psi_{\min }^{-1} K^{\prime}(t, \mathbf{x})+K_{\mathbf{v}}\right) \bar{K}(t, \mathbf{x}) \psi^{2} .
$$

This proves assertion (b) by the choice of $K_{1}$.

The next two propositions provide estimates for $H_{2}^{\psi}$ and $H_{3}$ in terms of the Euclidean distance between the reference feedback control $\boldsymbol{\zeta}^{0}(\Sigma)$ and alternatives $\boldsymbol{\zeta}$ :

Proposition 5.9 ( $H_{2}^{\psi}$ estimate). There is $K_{2}>0$ such that for every $(t, \mathbf{x}, y) \in \mathbf{D} \times \mathbb{R}, \boldsymbol{\zeta} \in \mathbf{Z}$, and $\psi>0$,

$$
\left|H_{2}^{\psi}(t, \mathbf{x}, y ; \boldsymbol{\zeta})\right| \leq K_{2}\left|\boldsymbol{\zeta}-\boldsymbol{\zeta}^{0}(\Sigma)\right|\left(\left|\boldsymbol{\zeta}-\boldsymbol{\zeta}^{0}(\Sigma)\right|+\frac{-U^{\prime \prime}(y)}{U^{\prime}(y)} \max \left(1,\left|\boldsymbol{\zeta}-\boldsymbol{\zeta}^{0}(\Sigma)\right|\right) \psi\right) .
$$


Proof. Set $K_{2}=\max \left(K_{\mathcal{V}}, 4 \max (1, \bar{\Sigma}) K_{\mathcal{V}} K_{\widetilde{w}}\right)$, where $K_{\mathcal{V}}$ and $K_{\widetilde{w}}$ are as in Assumption 4.2 (d) (e) Also fix $(t, \mathbf{x}, y) \in \mathbf{D} \times \mathbb{R}, \boldsymbol{\zeta} \in \mathbf{Z}$, and $\psi>0$. Now, first note that $b^{\mathcal{V}}(t, \mathbf{x} ; \boldsymbol{\zeta})$ is of the form (5.16) with

$$
\mathbf{a}=\left(\mathcal{V}_{\Sigma}, \beta \mathcal{V}_{A}+S^{2} \Gamma, S \frac{\partial \Delta}{\partial \Sigma}, \mathcal{V}_{\Sigma \Sigma}\right)^{\top}
$$

Hence, by Lemma 5.4 the fact that $|\mathbf{a}| \leq 2 K_{\mathcal{V}}$ by Assumption 4.2 (d), and the choice of $K_{2}$,

$$
\begin{aligned}
\left|b^{\mathcal{V}}(t, \mathbf{x} ; \boldsymbol{\zeta})\right| & \leq \max (1, \Sigma)|\mathbf{a}|\left|\boldsymbol{\zeta}-\boldsymbol{\zeta}^{0}(\Sigma)\right|+|\mathbf{a}|\left|\boldsymbol{\zeta}-\boldsymbol{\zeta}^{0}(\Sigma)\right|^{2} \\
& \leq \frac{K_{2}}{K_{\widetilde{w}}}\left|\boldsymbol{\zeta}-\boldsymbol{\zeta}^{0}(\Sigma)\right| \max \left(1,\left|\boldsymbol{\zeta}-\boldsymbol{\zeta}^{0}(\Sigma)\right|\right) .
\end{aligned}
$$

Next, using first norm estimates as in the proof of Proposition 5.9 (a) and then Assumption 4.2 (d) to estimate the resulting Frobenius norm by $2 K_{\mathcal{V}}$, we find

$$
\frac{1}{2}\left|\left(\begin{array}{c}
\sigma-\Sigma \\
\eta
\end{array}\right)^{\top}\left(\begin{array}{cc}
\beta \mathcal{V}_{A}+S^{2} \Gamma & S \frac{\partial \Delta}{\partial \Sigma} \\
S \frac{\partial \Delta}{\partial \Sigma} & \mathcal{V}_{\Sigma \Sigma}
\end{array}\right)\left(\begin{array}{c}
\sigma-\Sigma \\
\eta
\end{array}\right)\right| \leq K_{\mathcal{V}}\left|\boldsymbol{\zeta}-\boldsymbol{\zeta}^{0}(\Sigma)\right|^{2} .
$$

Finally, using (5.36)-(5.37) and the fact that $|\widetilde{w}| \leq K_{\widetilde{w}}$ on $\mathbf{D}$ by Assumption 4.2 (e), we obtain

$$
\begin{aligned}
\left|H_{2}^{\psi}(t, \mathbf{x}, y ; \boldsymbol{\zeta})\right| & \leq K_{\mathcal{V}}\left|\boldsymbol{\zeta}-\boldsymbol{\zeta}^{0}(\Sigma)\right|^{2}+K_{2} \frac{-U^{\prime \prime}(y)}{U^{\prime}(y)}\left|\boldsymbol{\zeta}-\boldsymbol{\zeta}^{0}(\Sigma)\right| \max \left(1,\left|\boldsymbol{\zeta}-\boldsymbol{\zeta}^{0}(\Sigma)\right|\right) \psi \\
& \leq K_{2}\left|\boldsymbol{\zeta}-\boldsymbol{\zeta}^{0}(\Sigma)\right|\left(\left|\boldsymbol{\zeta}-\boldsymbol{\zeta}^{0}(\Sigma)\right|+\frac{-U^{\prime \prime}(y)}{U^{\prime}(y)} \max \left(1,\left|\boldsymbol{\zeta}-\boldsymbol{\zeta}^{0}(\Sigma)\right|\right) \psi\right) .
\end{aligned}
$$

Proposition 5.10 ( $H_{3}$ estimate). There is $K_{3} \in L_{\mathfrak{P}}^{4}$ such that for every $(t, \mathbf{x}) \in \mathbf{D}$ and $\boldsymbol{\zeta} \in \mathbf{Z}$,

$$
\left|H_{3}(t, \mathbf{x} ; \boldsymbol{\zeta})-H_{3}\left(t, \mathbf{x} ; \boldsymbol{\zeta}^{0}(\Sigma)\right)\right| \leq K_{3}(t, \mathbf{x})\left|\boldsymbol{\zeta}-\boldsymbol{\zeta}^{0}(\Sigma)\right| \max \left(1,\left|\boldsymbol{\zeta}-\boldsymbol{\zeta}^{0}(\Sigma)\right|\right) .
$$

Proof. Set $K_{3}(t, \mathbf{x})=2 \max (1, \bar{\Sigma})|\mathbf{a}(t, \mathbf{x})|$ where

$$
\mathbf{a}(t, \mathbf{x})=\left(\widetilde{w}_{\Sigma}, \beta \widetilde{w}_{A}+S^{2}\left(\widetilde{w}_{S S}+2 \gamma \widetilde{w}_{S A}+\gamma^{2} \widetilde{w}_{A A}\right), S\left(\widetilde{w}_{S \Sigma}+\gamma \widetilde{w}_{A \Sigma}\right), \widetilde{w}_{\Sigma \Sigma}\right)^{\top} .
$$

By Assumption 4.2 (e), every component of $\mathbf{a}$ is in $L_{\mathfrak{P}}^{4}$ and thus also $K_{3} \in L_{\mathfrak{P}}^{4}$. Now, fix $(t, \mathbf{x}) \in \mathbf{D}$ and $\zeta \in \mathbf{Z}$. It is easy to see that the difference

$$
d:=H_{3}(t, \mathbf{x} ; \boldsymbol{\zeta})-H_{3}\left(t, \mathbf{x} ; \boldsymbol{\zeta}^{0}(\Sigma)\right)
$$

is of the form (5.16) for $\mathbf{a}=\mathbf{a}(t, \mathbf{x})$. Hence, by Lemma 5.4 and the choice of $K_{3}$,

$$
\begin{aligned}
|d| & \leq \max (1, \Sigma)|\mathbf{a}|\left|\boldsymbol{\zeta}-\boldsymbol{\zeta}^{0}(\Sigma)\right|+|\mathbf{a}|\left|\boldsymbol{\zeta}-\boldsymbol{\zeta}^{0}(\Sigma)\right|^{2} \\
& \leq K_{3}(t, \mathbf{x})\left|\boldsymbol{\zeta}-\boldsymbol{\zeta}^{0}(\Sigma)\right| \max \left(1,\left|\boldsymbol{\zeta}-\boldsymbol{\zeta}^{0}(\Sigma)\right|\right) .
\end{aligned}
$$

By combining Propositions 5.9 and 5.10 , the following corollary guarantees that if $\zeta$ is close to $\boldsymbol{\zeta}^{0}(\Sigma)$, then $H_{2}^{\psi}(t, \mathbf{x} ; \boldsymbol{\zeta})$ is of order $O\left(\psi^{2}\right)$ and $H_{3}(t, \mathbf{x} ; \boldsymbol{\zeta})$ can be replaced by $H_{3}\left(t, \mathbf{x} ; \boldsymbol{\zeta}^{0}(\Sigma)\right)$ and a term of order $O(\psi)$.

Corollary 5.11. Let $0 \leq K \in L_{\mathfrak{P}}^{4}$. There is $K_{2,3} \in L_{\mathfrak{P}}^{2}$ (depending on $K$ ) such that for every $(t, \mathbf{x}, y) \in \mathbf{D} \times \mathbb{R}, \boldsymbol{\zeta} \in \mathbf{Z}$, and $\psi>0$ satisfying

$$
\left|\boldsymbol{\zeta}-\boldsymbol{\zeta}^{0}(\Sigma)\right| \leq K(t, \mathbf{x}) \psi
$$

we have

$$
\begin{aligned}
\left|H_{2}^{\psi}(t, \mathbf{x}, y ; \boldsymbol{\zeta})\right| & \leq K_{2,3}(t, \mathbf{x})\left(1+\frac{-U^{\prime \prime}(y)}{U^{\prime}(y)}\right) \psi^{2} \\
\left|H_{3}(t, \mathbf{x} ; \boldsymbol{\zeta})-H_{3}\left(t, \mathbf{x} ; \boldsymbol{\zeta}^{0}(\Sigma)\right)\right| & \leq K_{2,3}(t, \mathbf{x}) \psi
\end{aligned}
$$


Proof. Choose $K_{2}>0$ and $K_{3} \in L_{\mathfrak{P}}^{4}$ as in Propositions $[5.9$ and $[5.10$. Since $\mathbf{Z}$ and $[\underline{\Sigma}, \bar{\Sigma}]$ are bounded, there is a constant $K^{\prime} \geq 1$ such that for every $\zeta \in \mathbf{Z}$ and $\Sigma \in[\underline{\Sigma}, \bar{\Sigma}]$,

$$
\left|\boldsymbol{\zeta}-\boldsymbol{\zeta}^{0}(\Sigma)\right| \leq K^{\prime}
$$

Set $K_{2,3}=K(t, \mathbf{x})\left(K_{2} \max \left(K^{\prime}, K(t, \mathbf{x})\right)+K_{3}(t, \mathbf{x}) K^{\prime}\right)$. It is easy to see that $K_{2,3} \in L_{\mathfrak{P}}^{2}$. Now, fix $(t, \mathbf{x}, y) \in \mathbf{D} \times \mathbb{R}, \boldsymbol{\zeta} \in \mathbf{Z}$, and $\psi>0$ satisfying (5.38). Then by Proposition 5.9 and (5.38),

$$
\left|H_{2}^{\psi}(t, \mathbf{x}, y ; \boldsymbol{\zeta})\right| \leq K_{2} K(t, \mathbf{x}) \psi\left(K(t, \mathbf{x}) \psi+\frac{-U^{\prime \prime}(y)}{U^{\prime}(y)} K^{\prime} \psi\right) \leq K_{2,3}(t, \mathbf{x})\left(1+\frac{-U^{\prime \prime}(y)}{U^{\prime}(y)}\right) \psi^{2} .
$$

Similarly, by Proposition 5.10 and (5.38),

$$
\left|H_{3}(t, \mathbf{x} ; \boldsymbol{\zeta})-H_{3}\left(t, \mathbf{x} ; \boldsymbol{\zeta}^{0}(\Sigma)\right)\right| \leq K_{3}(t, \mathbf{x}) K(t, \mathbf{x}) K^{\prime} \psi \leq K_{2,3}(t, \mathbf{x}) \psi .
$$

Finally, Proposition 5.12 below shows that $H_{4}^{\psi}$ is bounded from below by 0 up to a term of order $O\left(\psi^{2}\right)$. Recall from (5.21) that this asymptotic lower bound is attained by the delta-vega hedge, i.e., $H_{4}^{\psi}\left(t, \mathbf{x}, y ; \boldsymbol{v}^{\star}(t, \mathbf{x}), \boldsymbol{\zeta}\right)=0$.

Proposition $\mathbf{5 . 1 2}$ ( $H_{4}^{\psi}$ estimate). There is a nonnegative $K_{4} \in L_{\mathfrak{P}}^{2}$ such that for every $(t, \mathbf{x}, y) \in$ $\mathbf{D} \times \mathbb{R}, \boldsymbol{v} \in \mathbb{R}^{2}, \boldsymbol{\zeta} \in \mathbf{Z}$, and $\psi>0$,

$$
H_{4}^{\psi}(t, \mathbf{x}, y ; \boldsymbol{v}, \boldsymbol{\zeta}) \geq U^{\prime \prime}(y) K_{4}(t, \mathbf{x}) \psi^{2} .
$$

Proof. We first argue that $w_{Y Y}^{\psi} \leq U^{\prime \prime}$ on $\mathbf{D} \times \mathbb{R}$. As $U$ has decreasing absolute risk aversion (cf. Assumption 4.2](f)], we have for each $y \in \mathbb{R}$,

$$
0 \geq \frac{\mathrm{d}}{\mathrm{d} y}\left(-\frac{U^{\prime \prime}(y)}{U^{\prime}(y)}\right)=-\frac{U^{\prime}(y) U^{\prime \prime \prime}(y)-U^{\prime \prime}(y)^{2}}{U^{\prime}(y)^{2}} .
$$

In particular, since $U^{\prime}>0$, we have $U^{\prime \prime \prime}>0$. Together with $\widetilde{w} \geq 0$ (cf. Assumption $\left.4.2[\mathrm{e})\right]$, this yields $w_{Y Y}^{\psi}=U^{\prime \prime}-U^{\prime \prime \prime} \widetilde{w} \psi \leq U^{\prime \prime}<0$ on $\mathbf{D} \times \mathbb{R}$.

Now, set $K_{4}(t, \mathbf{x})=\frac{1}{2} K\left(\bar{\sigma}^{2} S^{2}\left(\widetilde{w}_{S}+\gamma \widetilde{w}_{A}\right)^{2}+\widetilde{w}_{\Sigma}^{2}\right)$ for some constant

$$
K \geq \max _{\boldsymbol{\zeta} \in \mathbf{Z}}\left(1+2 \eta^{2}+\left(\eta^{2}+\xi\right)^{2}\right)^{1 / 2} .
$$

As $S\left(\widetilde{w}_{S}+\gamma \widetilde{w}_{A}\right), \widetilde{w}_{\Sigma} \in L_{\mathfrak{P}}^{4}$ by Assumption 4.2 (e), we have $K_{4} \in L_{\mathfrak{P}}^{2}$. Next, fix $(t, \mathbf{x}, y) \in \mathbf{D} \times \mathbb{R}$, $\boldsymbol{\zeta} \in \mathbf{Z}$, and $\psi>0$. Write

$$
Q=\left(\begin{array}{cc}
1 & \eta \\
\eta & \eta^{2}+\xi
\end{array}\right) \quad \text { and } \quad \widetilde{\mathbf{w}}=\left(\begin{array}{c}
\sigma S\left(\widetilde{w}_{S}+\gamma \widetilde{w}_{A}\right) \\
\widetilde{w}_{\Sigma}
\end{array}\right)
$$

and consider the function $q: \mathbb{R}^{2} \rightarrow \mathbb{R}$ given by

$$
q(\mathbf{z})=-\frac{w_{Y Y}^{\psi}}{2} \mathbf{z}^{\top} Q \mathbf{z}+\psi U^{\prime \prime}(y) \widetilde{\mathbf{w}}^{\top} Q \mathbf{z} .
$$

Clearly, minimising $q$ over $\mathbf{z} \in \mathbb{R}^{2}$ is equivalent to minimising $H_{4}^{\psi}\left(t, \mathbf{x}, y ; \boldsymbol{v}, \boldsymbol{\zeta}\right.$ ) over $\boldsymbol{v} \in \mathbb{R}^{2}$ (recall that $\mathcal{C}_{\Sigma} \neq 0$ by Assumption 4.2 (c) . Moreover,

$$
\operatorname{det} Q=\left(\eta^{2}+\xi\right)-\eta^{2}=\xi \geq 0 \text { and } \operatorname{Trace} Q=1+\eta^{2}+\xi>0,
$$

so that the symmetric matrix $Q$ is positive semi-definite. It follows that $q$ is convex and any solution to the first-order condition

$$
Q\left(-w_{Y Y}^{\psi} \mathbf{z}+\psi U^{\prime \prime}(y) \widetilde{\mathbf{w}}\right)=0
$$


is a (global) minimiser. As $\mathbf{z}^{*}=\frac{U^{\prime \prime}(y)}{w_{Y Y}^{\psi}} \widetilde{\mathbf{w}} \psi$ solves the first-order condition, we obtain after some algebra that the minimum of $q$ is

$$
\frac{1}{2} \frac{U^{\prime \prime}(y)^{2}}{w_{Y Y}^{\psi}} \widetilde{\mathbf{w}}^{\top} Q \widetilde{\mathbf{w}} \psi^{2} .
$$

Using also that $w_{Y Y}^{\psi} \leq U^{\prime \prime}<0$ on $\mathbf{D} \times \mathbb{R}$, we conclude that for all $\boldsymbol{v} \in \mathbb{R}^{2}$,

$$
H_{4}^{\psi}(t, \mathbf{x}, y ; \boldsymbol{v}, \boldsymbol{\zeta}) \geq \frac{1}{2} U^{\prime \prime}(y) \widetilde{\mathbf{w}}^{\top} Q \widetilde{\mathbf{w}} \psi^{2} .
$$

Finally,

$$
\frac{1}{2}\left|\widetilde{\mathbf{w}}^{\top} Q \widetilde{\mathbf{w}}\right| \leq \frac{1}{2}|\widetilde{\mathbf{w}}|^{2}\|Q\|_{F} \leq K_{4}(t, \mathbf{x})
$$

by the choice of $K_{4}$. Combining the preceding two estimates completes the proof.

\subsubsection{Approximate solution to the HJBI equation}

The following lemma shows that the candidate value function $w^{\psi}$ defined in (5.2) is, up to a term of order $O\left(\psi^{2}\right)$, a "supersolution" to the HJBI equation (5.14). This analytic result is the main ingredient for the proof of the inequality (5.3) in Section 5.1.4

Lemma 5.13 (Lower bound). Fix constants $\underline{Y} \leq \bar{Y}$. There is a nonnegative $K_{\text {lo }} \in L_{\mathfrak{P}}^{1}$ (depending on $\underline{Y}, \bar{Y})$ such that for every $(t, \mathbf{x}, y) \in \mathbf{D} \times[\underline{Y}, \bar{Y}]$ and $\psi \in(0,1)$,

$$
w_{t}^{\psi}(t, \mathbf{x}, y)+\inf _{\boldsymbol{\zeta} \in \mathbf{Z}(t, \mathbf{x})} H^{\psi}\left(t, \mathbf{x}, y ; \boldsymbol{v}^{\star}(t, \mathbf{x}), \boldsymbol{\zeta}\right) \geq-K_{\mathrm{lo}}(t, \mathbf{x}) \psi^{2} .
$$

Proof. As an auxiliary result, we first prove that there is $K \in L_{\mathfrak{P}}^{4}$ such that for every $(t, \mathbf{x}, y) \in$ $\mathbf{D} \times[\underline{Y}, \bar{Y}], \boldsymbol{\zeta} \in \mathbf{Z}$, and $\psi \in(0,1)$ satisfying

$$
H^{\psi}\left(t, \mathbf{x}, y ; \boldsymbol{v}^{\star}(t, \mathbf{x}), \boldsymbol{\zeta}\right) \leq H^{\psi}\left(t, \mathbf{x}, y ; \boldsymbol{v}^{\star}(t, \mathbf{x}), \boldsymbol{\zeta}^{0}(\Sigma)\right),
$$

we have

$$
\left|\boldsymbol{\zeta}-\boldsymbol{\zeta}^{0}(\Sigma)\right| \leq K(t, \mathbf{x}) \psi .
$$

Using Proposition $\left[5.9\right.$ and the fact that $\mathbf{Z}$ and $[\underline{Y}, \bar{Y}]$ are compact, there is a constant $K_{2}^{\prime}>0$ such that for every $(t, \mathbf{x}, y) \in \mathbf{D} \times[\underline{Y}, \bar{Y}], \zeta \in \mathbf{Z}$, and $\psi \in(0,1)$,

$$
\left|H_{2}^{\psi}(t, \mathbf{x}, y ; \boldsymbol{\zeta})\right| \leq K_{2}^{\prime}\left|\boldsymbol{\zeta}-\boldsymbol{\zeta}^{0}(\Sigma)\right| \text {. }
$$

Similarly, using Proposition 5.10, there is $K_{3}^{\prime} \in L_{\mathfrak{P}}^{4}$ such that for every $(t, \mathbf{x}) \in \mathbf{D}$ and $\boldsymbol{\zeta} \in \mathbf{Z}$,

$$
\left|H_{3}(t, \mathbf{x} ; \boldsymbol{\zeta})-H_{3}\left(t, \mathbf{x} ; \boldsymbol{\zeta}^{0}(\Sigma)\right)\right| \leq K_{3}^{\prime}(t, \mathbf{x})\left|\boldsymbol{\zeta}-\boldsymbol{\zeta}^{0}(\Sigma)\right| .
$$

Now, set $K(t, \mathbf{x})=2 \psi_{\max }\left(|\mathbf{v}(t, \mathbf{x})|+K_{2}^{\prime}+K_{3}^{\prime}(t, \mathbf{x})\right)$. Using that $\mathbf{v}(t, \mathbf{x})$ is uniformly bounded by Assumption $4.2(\mathrm{~d})$, it follows that $K \in L_{\mathfrak{P}}^{4}$. Fix $(t, \mathbf{x}, y) \in \mathbf{D} \times[\underline{Y}, \bar{Y}], \boldsymbol{\zeta} \in \mathbf{Z}$, and $\psi \in(0,1)$ satisfying (5.40). Rearranging (5.40) and using the decomposition (5.18) of $H^{\psi}$, the fact that the $H_{4}^{\psi}$ term vanishes by (5.21), the above estimates for $H_{2}^{\psi}$ and $H_{3}$ as well as a direct estimate for the $H_{1}^{\psi}$ term, we find

$$
\begin{aligned}
0 & \geq\left(H^{\psi}\left(t, \mathbf{x}, y ; \boldsymbol{v}^{\star}(t, \mathbf{x}), \boldsymbol{\zeta}\right)-H^{\psi}\left(t, \mathbf{x}, y ; \boldsymbol{v}^{\star}(t, \mathbf{x}), \boldsymbol{\zeta}^{0}(\Sigma)\right)\right) / U^{\prime}(y) \\
& =H_{1}^{\psi}(t, \mathbf{x} ; \boldsymbol{\zeta})+H_{2}^{\psi}(t, \mathbf{x}, y ; \boldsymbol{\zeta})-\left(H_{3}(t, \mathbf{x} ; \boldsymbol{\zeta})-H_{3}\left(t, \mathbf{x} ; \boldsymbol{\zeta}^{0}(\Sigma)\right)\right) \psi \\
& \geq \frac{1}{2 \psi \psi_{\max }}\left|\boldsymbol{\zeta}-\boldsymbol{\zeta}^{0}(\Sigma)\right|^{2}-\mathbf{v}(t, \mathbf{x})^{\top}\left(\boldsymbol{\zeta}-\boldsymbol{\zeta}^{0}(\Sigma)\right)-\left(K_{2}^{\prime}+K_{3}^{\prime}(t, \mathbf{x})\right)\left|\boldsymbol{\zeta}-\boldsymbol{\zeta}^{0}(\Sigma)\right|
\end{aligned}
$$


By rearranging terms and applying the Cauchy-Schwarz inequality, we obtain

$$
\begin{aligned}
\left|\boldsymbol{\zeta}-\boldsymbol{\zeta}^{0}(\Sigma)\right|^{2} & \leq 2 \psi_{\max }\left(|\mathbf{v}(t, \mathbf{x})|+K_{2}^{\prime}+K_{3}^{\prime}(t, \mathbf{x})\right)\left|\boldsymbol{\zeta}-\boldsymbol{\zeta}^{0}(\Sigma)\right| \psi \\
& \leq K(t, \mathbf{x})\left|\boldsymbol{\zeta}-\boldsymbol{\zeta}^{0}(\Sigma)\right| \psi
\end{aligned}
$$

and (5.41) follows.

We now turn to the proof of (5.39). Choose $K_{2,3} \in L_{\mathfrak{P}}^{2}$ as in Corollary 5.11) (with $K$ as in the auxiliary result), $K_{1} \in L_{\mathfrak{P}}^{1}$ as in Proposition 5.8 (a), and set

$$
K_{\mathrm{lo}}(t, \mathbf{x})=U^{\prime}(\underline{Y})\left(K_{1}(t, \mathbf{x})+K_{2,3}(t, \mathbf{x})\left(2+\frac{-U^{\prime \prime}(\underline{Y})}{U^{\prime}(\underline{Y})}\right)\right) .
$$

Clearly, $K_{\text {lo }} \in L_{\mathfrak{P}}^{1}$. Fix $(t, \mathbf{x}, y) \in \mathbf{D} \times[\underline{Y}, \bar{Y}], \zeta \in \mathbf{Z}(t, \mathbf{x})$, and $\psi \in(0,1)$. First, we note that $\zeta^{0}(\Sigma) \in \mathbf{Z}(t, \mathbf{x})$ and that by (5.20)-(5.21) and Lemma $5.6(\mathrm{c})$,

$$
\begin{aligned}
w_{t}^{\psi}(t, \mathbf{x}, y)+H^{\psi}\left(t, \mathbf{x}, y ; \boldsymbol{v}^{\star}(t, \mathbf{x}), \boldsymbol{\zeta}^{0}(\Sigma)\right) & =-U^{\prime}(y)\left(\widetilde{w}_{t}(t, \mathbf{x})+H_{3}\left(t, \mathbf{x} ; \boldsymbol{\zeta}^{0}(\Sigma)\right)\right) \psi \\
& =\frac{1}{2} U^{\prime}(y) \widetilde{g}(t, \mathbf{x}) \psi \geq 0 .
\end{aligned}
$$

In view of assertion (5.39), we may thus assume that (5.40) is satisfied. In turn, (5.41) holds by the auxiliary result. In particular, we may use the estimates of Proposition 5.8 (a) (for $H_{1}^{\psi}$ ) and Corollary 5.11 (for $H_{2}^{\psi}$ and $H_{3}$ ) in the following. These together with the fact that the $H_{4}^{\psi}$ term vanishes by (5.21) yield

$$
\begin{aligned}
w_{t}^{\psi}(t, \mathbf{x}, y)+H^{\psi}\left(t, \mathbf{x}, y ; \boldsymbol{v}^{\star}(t, \mathbf{x}), \boldsymbol{\zeta}\right) \\
=U^{\prime}(y)\left(-\widetilde{w}_{t}(t, \mathbf{x}) \psi+H_{1}^{\psi}(t, \mathbf{x} ; \boldsymbol{\zeta})+H_{2}^{\psi}(t, \mathbf{x}, y ; \boldsymbol{\zeta})-H_{3}(t, \mathbf{x} ; \boldsymbol{\zeta}) \psi\right) \\
\geq-U^{\prime}(y)\left(\widetilde{w}_{t}(t, \mathbf{x})+H_{3}\left(t, \mathbf{x} ; \boldsymbol{\zeta}^{0}(\Sigma)\right)+\frac{1}{2} \widetilde{g}(t, \mathbf{x})\right) \psi \\
\quad-U^{\prime}(y)\left(K_{1}(t, \mathbf{x})+K_{2,3}(t, \mathbf{x})\left(1+\frac{-U^{\prime \prime}(y)}{U^{\prime}(y)}\right)+K_{2,3}(t, \mathbf{x})\right) \psi^{2} \\
\geq-K_{\mathrm{lo}}(t, \mathbf{x}) \psi^{2}
\end{aligned}
$$

where in the last inequality, we also use (5.20) to eliminate the $O(\psi)$ term and the fact that $U$ has decreasing absolute risk aversion (cf. Assumption $4.2(\mathrm{f})$ to estimate the $O\left(\psi^{2}\right)$ term. As $\boldsymbol{\zeta} \in \mathbf{Z}(t, \mathbf{x})$ was arbitrary, (5.39) follows.

Conversely, the next lemma shows that the candidate value function $w^{\psi}$ defined in (5.2) is asymptotically a "subsolution" to the HJBI equation (5.14). Here, the asymptotic estimate is of order $O\left(\psi^{2}\right)$ if $\Sigma$ is in the interior of $[\underline{\Sigma}, \bar{\Sigma}]$ and of order $O(\psi)$ otherwise. This analytic result is the main ingredient for the proof of the inequality (5.4) in Section 5.1.5.

Lemma 5.14 (Upper bound). Let $0 \leq \bar{K} \in L_{\mathfrak{P}}^{4}$. There is a nonnegative $K_{\mathrm{up}} \in L_{\mathfrak{P}}^{2}$ (depending on $\bar{K})$ such that for every $(t, \mathbf{x}, y) \in \mathbf{D} \times \mathbb{R}, \zeta \in \mathbf{Z}$, and $\psi \in(0,1)$ satisfying

$$
\left|\boldsymbol{\zeta}-\boldsymbol{\zeta}^{\psi}(t, \mathbf{x})\right| \leq \bar{K}(t, \mathbf{x}) \psi^{2},
$$

we have

$$
w_{t}^{\psi}(t, \mathbf{x}, y)+\sup _{\boldsymbol{v} \in \mathbb{R}^{2}} H^{\psi}(t, \mathbf{x}, y ; \boldsymbol{v}, \boldsymbol{\zeta}) \leq K_{\mathrm{up}}(t, \mathbf{x}) U^{\prime}(y)\left(1+\frac{-U^{\prime \prime}(y)}{U^{\prime}(y)}\right) \psi^{1+\mathbf{1}_{\{\Sigma \in(\underline{\Sigma}, \bar{\Sigma})\}}} .
$$

Proof. Define $K_{\boldsymbol{\zeta}} \geq 1$ as in Lemma $5.6(\mathrm{~d})$, and set $K(t, \mathbf{x})=\bar{K}(t, \mathbf{x})+K_{\boldsymbol{\zeta}}$. Clearly, $K \in L_{\mathfrak{P}}^{4}$. With this choice of $K$, let $K_{2,3} \in L_{\mathfrak{P}}^{2}$ be defined as in Corollary [5.11. Moreover, define $K_{1} \in L_{\mathfrak{P}}^{2}$ 
as in Proposition 5.8 (b) and $K_{4} \in L_{\mathfrak{P}}^{2}$ as in Proposition 5.12, In addition, note that there is $K_{\widetilde{g}}>0$ such that $0 \leq \widetilde{g} \leq K_{\widetilde{g}}$ on $\mathbf{D}$ by Lemma $5.6(\mathrm{c})$.

Now, set $K_{\text {up }}(t, \mathbf{x})=4 \max \left(K_{1}(t, \mathbf{x})+2 K_{2,3}(t, \mathbf{x})+K_{4}(t, \mathbf{x}), \frac{1}{2} K_{\widetilde{g}}\right)$. Clearly, $K_{\text {up }} \in L_{\mathfrak{P}}^{2}$. Fix $(t, \mathbf{x}, y) \in \mathbf{D} \times \mathbb{R}, \boldsymbol{v} \in \mathbb{R}^{2}, \boldsymbol{\zeta} \in \mathbf{Z}$, and $\psi \in(0,1)$ satisfying (5.42). In particular, condition (5.33) of Proposition 5.8 (b) holds. By Lemma 5.6 (d) and (5.42),

$$
\left|\boldsymbol{\zeta}-\boldsymbol{\zeta}^{0}(\Sigma)\right| \leq\left|\boldsymbol{\zeta}-\boldsymbol{\zeta}^{\psi}(t, \mathbf{x})\right|+\left|\boldsymbol{\zeta}^{\psi}(t, \mathbf{x})-\boldsymbol{\zeta}^{0}(\Sigma)\right| \leq \bar{K}(t, \mathbf{x}) \psi^{2}+K_{\boldsymbol{\zeta}} \psi \leq K(t, \mathbf{x}) \psi,
$$

so that condition (5.38) of Corollary 5.11] is satisfied as well.

Using Propositions 5.8 (b) (for $H_{1}^{\psi}$ ) and 5.12 (for $H_{4}^{\psi}$ ) as well as Corollary 5.11 (for $H_{2}^{\psi}$ and $H_{3}$ ) to estimate the four summands in the decomposition (5.18) of $H^{\psi}$, and also (5.20) in the penultimate step, we obtain

$$
\begin{aligned}
& w_{t}^{\psi}(t, \mathbf{x}, y)+H^{\psi}(t, \mathbf{x}, y ; \boldsymbol{v}, \boldsymbol{\zeta}) \\
& \leq-U^{\prime}(y) \widetilde{w}_{t}(t, \mathbf{x}) \psi+U^{\prime}(y)\left(-\frac{1}{2} \widetilde{g}(t, \mathbf{x}) \mathbf{1}_{\{\Sigma \in(\underline{\Sigma}, \bar{\Sigma})\}} \psi+K_{1}(t, \mathbf{x}) \psi^{2}\right) \\
& +U^{\prime}(y) K_{2,3}(t, \mathbf{x})\left(1+\frac{-U^{\prime \prime}(y)}{U^{\prime}(y)}\right) \psi^{2} \\
& -U^{\prime}(y)\left(H_{3}\left(t, \mathbf{x} ; \boldsymbol{\zeta}^{0}(\Sigma)\right)-K_{2,3}(t, \mathbf{x}) \psi\right) \psi-U^{\prime \prime}(y) K_{4}(t, \mathbf{x}) \psi^{2} \\
& =-U^{\prime}(y)\left(\widetilde{w}_{t}(t, \mathbf{x})+H_{3}\left(t, \mathbf{x} ; \boldsymbol{\zeta}^{0}(\Sigma)\right)+\frac{1}{2} \widetilde{g}(t, \mathbf{x}) \mathbf{1}_{\{\Sigma \in(\underline{\Sigma}, \bar{\Sigma})\}}\right) \psi \\
& +U^{\prime}(y)\left(K_{1}(t, \mathbf{x})+2 K_{2,3}(t, \mathbf{x})+\left(K_{2,3}(t, \mathbf{x})+K_{4}(t, \mathbf{x})\right) \frac{-U^{\prime \prime}(y)}{U^{\prime}(y)}\right) \psi^{2} \\
& \leq U^{\prime}(y) \frac{1}{2} \widetilde{g}(t, \mathbf{x}) \mathbf{1}_{\{\Sigma \in\{\underline{\Sigma}, \bar{\Sigma}\}\}} \psi+\frac{1}{4} K_{\text {up }}(t, \mathbf{x}) U^{\prime}(y)\left(1+\frac{-U^{\prime \prime}(y)}{U^{\prime}(y)}\right) \psi^{2} \\
& \leq \frac{1}{2} K_{\mathrm{up}}(t, \mathbf{x}) U^{\prime}(y)\left(1+\frac{-U^{\prime \prime}(y)}{U^{\prime}(y)}\right)\left(\mathbf{1}_{\{\Sigma \in\{\underline{\Sigma}, \bar{\Sigma}\}\}} \psi+\psi^{2}\right) .
\end{aligned}
$$

As $\boldsymbol{v} \in \mathbb{R}^{2}$ was arbitrary, the assertion follows easily by distinguishing the cases $\Sigma \in(\underline{\Sigma}, \bar{\Sigma})$ and $\Sigma \in\{\underline{\Sigma}, \bar{\Sigma}\}$ (using that $\psi \in(0,1)$ in the second case).

\subsubsection{The asymptotic lower bound for the stochastic differential game}

We are now in a position to establish an asymptotic lower bound for the SDG (2.16), as required for the proof of Theorem 4.5 at the beginning of Section 5.1 .

Lemma 5.15. As $\psi \downarrow 0$,

$$
\inf _{P \in \mathfrak{P}^{3}} J^{\psi}\left(\boldsymbol{v}^{\star}, P\right) \geq w_{0}^{\psi}+o(\psi) .
$$

Proof. Choose $\underline{Y}, \bar{Y}$ as in Corollary 5.3 and, with this choice, let $K_{\mathrm{lo}} \in L_{\mathfrak{P}}^{1}$ be as in Lemma 5.13 , Now, fix $\varepsilon>0, \psi_{0}^{\prime} \in\left(0, \psi_{0}\right)$ such that $\left\|K_{\mathrm{lo}}\right\|_{L_{\mathfrak{P}}^{1}} \psi_{0}^{\prime} \leq \frac{1}{2} \varepsilon$, and let $\psi \in\left(0, \psi_{0}^{\prime}\right)$. We need to show that

$$
\inf _{P^{\prime} \in \mathfrak{P}} J^{\psi}\left(\boldsymbol{v}^{\star}, P^{\prime}\right)-w_{0}^{\psi} \geq-\varepsilon \psi
$$

Choose $P \in \mathfrak{P}$ such that $J^{\psi}\left(\boldsymbol{v}^{\star}, P\right)-\frac{1}{2} \varepsilon \psi \leq \inf _{P^{\prime} \in \mathfrak{P}} J^{\psi}\left(\boldsymbol{v}^{\star}, P^{\prime}\right)$. Then

$$
\inf _{P^{\prime} \in \mathfrak{P}} J^{\psi}\left(\boldsymbol{v}^{\star}, P^{\prime}\right)-w_{0}^{\psi} \geq J^{\psi}\left(\boldsymbol{v}^{\star}, P\right)-w_{0}^{\psi}-\frac{1}{2} \varepsilon \psi .
$$


Applying Itô's formula (under $P$ ) to the process $w^{\psi}\left(u, \mathbf{X}_{u}, Y_{u}^{v^{\star}, P}\right)$ (recall the dynamics of $S, A, M, \Sigma$, and $Y^{\boldsymbol{v}^{\star}, P}$ given in (2.6)-(2.8) and Corollary [5.3) and using the third line in (4.7) (so that the $\mathrm{d} M$-integral vanishes) yields for each $u \in[0, T]$,

$$
\begin{aligned}
I_{u}^{\psi}\left(\boldsymbol{v}^{\star}, P\right) & :=w^{\psi}\left(u, \mathbf{X}_{u}, Y_{u}^{\boldsymbol{v}^{\star}, P}\right)+\frac{1}{\psi} \int_{0}^{u} U^{\prime}\left(Y_{t}^{\boldsymbol{v}^{\star}, P}\right) f\left(\Sigma_{t}, \boldsymbol{\zeta}_{t}^{P}\right) \mathrm{d} t-w_{0}^{\psi} \\
& =N_{u}+\int_{0}^{u}\left(w_{t}^{\psi}\left(t, \mathbf{X}_{t}, Y_{t}^{\boldsymbol{v}^{\star}, P}\right)+H^{\psi}\left(t, \mathbf{X}_{t}, Y_{t}^{\boldsymbol{v}^{\star}, P} ; \boldsymbol{v}^{\star}\left(t, \mathbf{X}_{t}\right), \boldsymbol{\zeta}_{t}^{P}\right)\right) \mathrm{d} t,
\end{aligned}
$$

where

$$
\begin{aligned}
N:= & \int_{0}\left(w_{S}^{\psi}\left(t, \mathbf{X}_{t}, Y_{t}^{\boldsymbol{v}^{\star}, P}\right)+\gamma\left(t, S_{t}, A_{t}, M_{t}\right) w_{A}^{\psi}\left(t, \mathbf{X}_{t}, Y_{t}^{\boldsymbol{v}^{\star}, P}\right)\right) \mathrm{d} S_{t} \\
& +\int_{0} w_{\Sigma}^{\psi}\left(t, \mathbf{X}_{t}, Y_{t}^{\boldsymbol{v}^{\star}, P}\right) \mathrm{d} \Sigma_{t}^{c, P} .
\end{aligned}
$$

Note that $\boldsymbol{\zeta}_{t}^{P} \in \mathbf{Z}\left(t, \mathbf{X}_{t}\right) \mathrm{d} t \times P$-a.e. by (2.7). Hence, by Lemma 5.13, for each $u \in[0, T]$,

$$
I_{u}^{\psi}\left(\boldsymbol{v}^{\star}, P\right) \geq N_{u}-\int_{0}^{u} K_{\mathrm{lo}}\left(t, \mathbf{X}_{t}\right) \mathrm{d} t \psi^{2} .
$$

By construction, $N$ is a local $P$-martingale starting in 0 . Suppose for the moment that $N$ is also a submartingale. Then by taking expectations under $P$ on both sides of (5.47) (for $u=T$ ), we obtain

$$
J^{\psi}\left(\boldsymbol{v}^{\star}, P\right)-w_{0}^{\psi} \geq-\left\|K_{\mathrm{lo}}\right\|_{L_{\mathfrak{P}}^{1}} \psi^{2} \geq-\frac{1}{2} \varepsilon \psi .
$$

Combining this with (5.45) yields (5.44).

It remains to show that $N$ is a submartingale under $P$. As it is a local martingale, it suffices to show that it is bounded from above by a $P$-integrable random variable. To this end, first note from the definition of $w^{\psi}$ in (5.2), the fact that $\widetilde{w} \geq 0$ on $\mathbf{D}$ by Assumption 4.2 (e), and Assumption 4.2 (f) that $w^{\psi} \leq U(\bar{Y})$ on $\mathbf{D} \times[\underline{Y}, \bar{Y}]$. Clearly, $U^{\prime}(y) f(\Sigma, \boldsymbol{\zeta})$ is also uniformly bounded over $y \geq \underline{Y}, \Sigma \in[\underline{\Sigma}, \bar{\Sigma}]$, and $\boldsymbol{\zeta} \in \mathbf{Z}$. In view of the definition of $I^{\psi}\left(\boldsymbol{v}^{\star}, P\right)$ in (5.46), the fact that $Y^{\boldsymbol{v}^{\star}, P} \in[\underline{\underline{Y}}, \bar{Y}] \mathrm{d} t \times P$-a.e. by Corollary [5.3, and Assumption 4.2 (b), we conclude that $I^{\psi}\left(\boldsymbol{v}^{\star}, P\right) \leq K_{I} \mathrm{~d} t \times P$-a.e. for some constant $K_{I}>0$. Using this and (5.47), we obtain for each $u \in[0, T]$,

$$
N_{u} \leq K_{I}+\int_{0}^{T} K_{\mathrm{lo}}\left(t, \mathbf{X}_{t}\right) \mathrm{d} t
$$

As $K_{\text {lo }} \in L_{\mathfrak{P}}^{1}, N$ is bounded from above by a $P$-integrable random variable and therefore is a submartingale. This completes the proof.

\subsubsection{The asymptotic upper bound for the stochastic differential game}

To establish an asymptotic upper bound for the stochastic differential game (2.16), we first prove that the probability under $P^{\psi}$ that $\Sigma$ leaves $(\underline{\Sigma}, \bar{\Sigma})$ before time $T$ is of order $O(\psi)$.

Proposition 5.16. Let $\tau:=\inf \left\{t \in[0, T]: \Sigma_{t} \notin(\underline{\Sigma}, \bar{\Sigma})\right\} \wedge T$ be the first time that $\Sigma$ leaves $(\underline{\Sigma}, \bar{\Sigma})$. Then $\tau$ is a stopping time and there is $K_{\tau}>0$ such that for every $\psi \in\left(0, \psi_{0}\right)$,

$$
P^{\psi}[\tau<T] \leq K_{\tau} \psi
$$

Proof. It is an easy exercise to show that $\tau$ is a stopping time for the (non-augmented, non-rightcontinuous) filtration $\mathbb{F}$. This uses the fact that all paths of $\Sigma$ are continuous and $(\underline{\Sigma}, \bar{\Sigma})$ is open; cf. [38, Problem 2.7 in Chapter 1]. 
Turning to the proof of (5.48), by standard estimates for Itô processes (cf., e.g., [51, Lemma V.11.5]), there is a constant $K>0$ (depending only on $T$ ) such that for every $\psi \in\left(0, \psi_{0}\right)$,

$$
E^{P^{\psi}}\left[\sup _{0 \leq t \leq T}\left|\Sigma_{t}-\Sigma_{0}\right|^{2}\right] \leq K E^{P^{\psi}}\left[\int_{0}^{T}\left(\left(\nu_{t}^{P^{\psi}}\right)^{2}+\left(\eta_{t}^{P^{\psi}}\right)^{2}+\xi_{t}^{P^{\psi}}\right) \mathrm{d} t\right] .
$$

Define $K_{\zeta} \geq 1$ as in Lemma $5.6(\mathrm{~d})$, and let $K_{0}^{\prime}(t, \mathbf{x})=K_{0}(t, \mathbf{x})+K_{\zeta} \geq 1$ as well as $K_{\tau}=$ $2 \ell^{-2} K\left\|K_{0}^{\prime}\right\|_{L_{\mathfrak{P}}^{2}}^{2}$ with $\ell:=\min \left(\bar{\Sigma}-\Sigma_{0}, \Sigma_{0}-\underline{\Sigma}\right)>0$. Clearly, $K_{0}^{\prime} \in L_{\mathfrak{P}}^{4} \subset L_{\mathfrak{P}}^{2}$, so that $0 \leq K_{\tau}<\infty$. Fix $\psi \in\left(0, \psi_{0}\right)$. By (5.1) and Lemma 5.6 (d),

$$
\begin{aligned}
\left|\boldsymbol{\zeta}_{t}^{P^{\psi}}-\boldsymbol{\zeta}^{0}\left(\Sigma_{t}\right)\right| & \leq\left|\boldsymbol{\zeta}_{t}^{P^{\psi}}-\boldsymbol{\zeta}^{\psi}\left(t, \mathbf{X}_{t}\right)\right|+\left|\boldsymbol{\zeta}^{\psi}\left(t, \mathbf{X}_{t}\right)-\boldsymbol{\zeta}^{0}\left(\Sigma_{t}\right)\right| \leq K_{0}\left(t, \mathbf{X}_{t}\right) \psi^{2}+K_{\boldsymbol{\zeta}} \psi \\
& \leq K_{0}^{\prime}\left(t, \mathbf{X}_{t}\right) \psi \quad \mathrm{d} t \times P^{\psi} \text {-a.e. }
\end{aligned}
$$

Recalling that $\boldsymbol{\zeta}^{0}(\Sigma)=(0, \Sigma, 0,0)^{\top}$, this estimate yields

$$
\begin{aligned}
\left(\nu_{t}^{P^{\psi}}\right)^{2}+\left(\eta_{t}^{P^{\psi}}\right)^{2}+\xi_{t}^{P^{\psi}} & \leq\left|\boldsymbol{\zeta}_{t}^{P^{\psi}}-\boldsymbol{\zeta}^{0}\left(\Sigma_{t}\right)\right|^{2}+\left|\boldsymbol{\zeta}_{t}^{P^{\psi}}-\boldsymbol{\zeta}^{0}\left(\Sigma_{t}\right)\right| \leq K_{0}^{\prime}\left(t, \mathbf{X}_{t}\right)^{2} \psi^{2}+K_{0}^{\prime}\left(t, \mathbf{X}_{t}\right) \psi \\
& \leq 2 K_{0}^{\prime}\left(t, \mathbf{X}_{t}\right)^{2} \psi \quad \mathrm{d} t \times P^{\psi} \text {-a.e. }
\end{aligned}
$$

Moreover, by the definition of $\ell$ and Markov's inequality,

$$
P^{\psi}[\tau<T] \leq P^{\psi}\left[\sup _{0 \leq t \leq T}\left|\Sigma_{t}-\Sigma_{0}\right|^{2} \geq \ell^{2}\right] \leq \ell^{-2} E^{P^{\psi}}\left[\sup _{0 \leq t \leq T}\left|\Sigma_{t}-\Sigma_{0}\right|^{2}\right] .
$$

Combining (5.49)-(5.51) proves (5.48).

We are now in a position to establish an asymptotic upper bound for the stochastic differential game (2.16), which completes the proof of Theorem 4.5 at the beginning of Section 5.1.

Lemma 5.17. As $\psi \downarrow 0$,

$$
\sup _{\boldsymbol{v} \in \mathfrak{Y}} J^{\psi}\left(\boldsymbol{v}, P^{\psi}\right) \leq w_{0}^{\psi}+o(\psi) .
$$

Proof. Set $\bar{K}=K_{0} \in L_{\mathfrak{P}}^{4}$. With this choice of $\bar{K}$, define $K_{\text {up }} \in L_{\mathfrak{P}}^{2}$ as in Lemma 5.14. As $U^{\prime}$ is decreasing and $U$ has decreasing absolute risk aversion (cf. Assumption 4.2 (f)], there is $K_{U}>0$ such that (cf. Assumption 4.2 (a) for the choice of $K_{\mathfrak{Y}}$ )

$$
U^{\prime}(y)\left(1-U^{\prime \prime}(y) / U^{\prime}(y)\right) \leq K_{U}, \quad \text { for all } y \geq-K_{\mathfrak{Y}} .
$$

Now, fix $\varepsilon>0$, choose $\psi_{0}^{\prime} \in\left(0, \psi_{0}\right)$ such that

$$
K_{U}\left\|K_{\mathrm{up}}\right\|_{L_{\mathfrak{P}}^{1}} \psi_{0}^{\prime}+K_{U} \sqrt{T}\left\|K_{\mathrm{up}}\right\|_{L_{\mathfrak{P}}^{2}} K_{\tau}^{1 / 2}\left(\psi_{0}^{\prime}\right)^{1 / 2} \leq \frac{1}{2} \varepsilon
$$

where $K_{\tau}>0$ is as in Proposition [5.16, and let $\psi \in\left(0, \psi_{0}^{\prime}\right)$. We need to show that

$$
\sup _{\boldsymbol{v}^{\prime} \in \mathfrak{Y}} J^{\psi}\left(\boldsymbol{v}^{\prime}, P^{\psi}\right)-w_{0}^{\psi} \leq \varepsilon \psi .
$$

Choose $\boldsymbol{v} \in \mathfrak{Y}$ such that $J^{\psi}\left(\boldsymbol{v}, P^{\psi}\right)+\frac{1}{2} \varepsilon \psi \geq \sup _{\boldsymbol{v}^{\prime} \in \mathfrak{Y}} J^{\psi}\left(\boldsymbol{v}^{\prime}, P^{\psi}\right)$. Then

$$
\sup _{\boldsymbol{v}^{\prime} \in \mathfrak{Y}} J^{\psi}\left(\boldsymbol{v}^{\prime}, P^{\psi}\right)-w_{0}^{\psi} \leq J^{\psi}\left(\boldsymbol{v}, P^{\psi}\right)-w_{0}^{\psi}+\frac{1}{2} \varepsilon \psi \text {. }
$$


Applying Itô's formula (under $\left.P^{\psi}\right)$ to the process $w^{\psi}\left(u, \mathbf{X}_{u}, Y_{u}^{\boldsymbol{v}, P^{\psi}}\right)$ (recall the dynamics of $S, A, M, \Sigma$, and $Y^{\boldsymbol{v}, P^{\psi}}$ given in (2.6) (2.8) and Lemma 5.2) and using the third line in (4.7) (so that the $\mathrm{d} M$-integral vanishes) yields for each $u \in[0, T]$,

$$
\begin{aligned}
I_{u}^{\psi}\left(\boldsymbol{v}, P^{\psi}\right) & :=w^{\psi}\left(u, \mathbf{X}_{u}, Y_{u}^{\boldsymbol{v}, P^{\psi}}\right)+\frac{1}{\psi} \int_{0}^{u} U^{\prime}\left(Y_{t}^{\boldsymbol{v}, P^{\psi}}\right) f\left(\Sigma_{t}, \boldsymbol{\zeta}_{t}^{P^{\psi}}\right) \mathrm{d} t-w_{0}^{\psi} \\
& =N_{u}+\int_{0}^{u}\left(w_{t}^{\psi}\left(t, \mathbf{X}_{t}, Y_{t}^{\boldsymbol{v}, P^{\psi}}\right)+H^{\psi}\left(t, \mathbf{X}_{t}, Y_{t}^{\boldsymbol{v}, P^{\psi}} ; \boldsymbol{v}_{t}, \boldsymbol{\zeta}_{t}^{P^{\psi}}\right)\right) \mathrm{d} t .
\end{aligned}
$$

Here,

$$
\begin{aligned}
N:= & \int_{0}\left(w_{S}^{\psi}\left(t, \mathbf{X}_{t}, Y_{t}^{\boldsymbol{v}, P^{\psi}}\right)+\gamma\left(t, S_{t}, A_{t}, M_{t}\right) w_{A}^{\psi}\left(t, \mathbf{X}_{t}, Y_{t}^{\boldsymbol{v}, P^{\psi}}\right)\right) \mathrm{d} S_{t} \\
& +\int_{0} w_{\Sigma}^{\psi}\left(t, \mathbf{X}_{t}, Y_{t}^{\boldsymbol{v}, P^{\psi}}\right) \mathrm{d} \Sigma_{t}^{c, P^{\psi}}+\int_{0} w_{Y}^{\psi}\left(t, \mathbf{X}_{t}, Y_{t}^{\boldsymbol{v}, P^{\psi}}\right) \mathrm{d} Y_{t}^{c, \boldsymbol{v}, P^{\psi}}
\end{aligned}
$$

and $Y^{c, \boldsymbol{v}, P^{\psi}}=Y^{\boldsymbol{v}, P^{\psi}}+\int_{0}^{\cdot} b^{\mathcal{V}}\left(t, \mathbf{X}_{t} ; \boldsymbol{\zeta}_{t}^{P^{\psi}}\right) \mathrm{d} t$ is the local martingale part of $Y^{\boldsymbol{v}, P^{\psi}}$ under $P^{\psi}$.

We want to use Lemma 5.14 to estimate the drift term in the last line of (5.55). Note that condition (5.42) of Lemma 5.14 with $\boldsymbol{\zeta}$ and $\mathbf{x}$ replaced by $\boldsymbol{\zeta}_{t}^{P^{\psi}}$ and $\mathbf{X}_{t}$, respectively, is fulfilled $\mathrm{d} t \times P^{\psi}$-a.e. by (5.1) and our choice of $\bar{K}=K_{0}$. Moreover, denoting by $\tau$ the first time that $\Sigma$ leaves $(\underline{\Sigma}, \bar{\Sigma})$ (cf. Proposition $[5.16)$, we have for each $t \in[0, T)$ that $\Sigma_{t} \in(\underline{\Sigma}, \bar{\Sigma}) P^{\psi}$-a.s. on $\{\tau \geq T\}$. Therefore, by (5.43), (5.52), and Assumption 4.2 (a), for each $u \in[0, T]$,

$$
\begin{aligned}
I_{u}^{\psi}\left(\boldsymbol{v}, P^{\psi}\right) & \leq N_{u}+\int_{0}^{u} K_{\mathrm{up}}\left(t, \mathbf{X}_{t}\right) U^{\prime}\left(Y_{t}^{\boldsymbol{v}, P^{\psi}}\right)\left(1+\frac{-U^{\prime \prime}\left(Y_{t}^{\boldsymbol{v}, P^{\psi}}\right)}{U^{\prime}\left(Y_{t}^{\boldsymbol{v}, P^{\psi}}\right)}\right) \mathrm{d} t\left(\psi^{2} \mathbf{1}_{\{\tau \geq T\}}+\psi \mathbf{1}_{\{\tau<T\}}\right) \\
& \leq N_{u}+K_{U} \int_{0}^{u} K_{\mathrm{up}}\left(t, \mathbf{X}_{t}\right) \mathrm{d} t\left(\psi^{2} \mathbf{1}_{\{\tau \geq T\}}+\psi \mathbf{1}_{\{\tau<T\}}\right) .
\end{aligned}
$$

By construction, $N$ is a local $P^{\psi}$-martingale starting in 0 . Suppose for the moment that $N$ is also a supermartingale. Then by taking expectations under $P^{\psi}$ on both sides of (5.56) (for $u=T$ ) and using the Cauchy-Schwarz and Jensen inequalities as well as Proposition 5.16, we obtain

$$
\begin{aligned}
J^{\psi}\left(\boldsymbol{v}, P^{\psi}\right)-w_{0}^{\psi} & \leq K_{U}\left\|K_{\mathrm{up}}\right\|_{L_{\mathfrak{q}}^{1}} \psi^{2}+K_{U} E^{P^{\psi}}\left[\int_{0}^{T} K_{\mathrm{up}}\left(t, \mathbf{X}_{t}\right) \mathrm{d} t \mathbf{1}_{\{\tau<T\}}\right] \psi \\
& \leq K_{U}\left\|K_{\mathrm{up}}\right\|_{L_{\mathfrak{q}}^{1}} \psi^{2}+K_{U} E^{P^{\psi}}\left[\left(\int_{0}^{T} K_{\mathrm{up}}\left(t, \mathbf{X}_{t}\right) \mathrm{d} t\right)^{2}\right]^{1 / 2} P^{\psi}[\tau<T]^{1 / 2} \psi \\
& \leq\left(K_{U}\left\|K_{\mathrm{up}}\right\|_{L_{\mathfrak{q}}^{1}} \psi+K_{U} \sqrt{T}\left\|K_{\mathrm{up}}\right\|_{L_{\mathfrak{q}}^{2}} K_{\tau}^{1 / 2} \psi^{1 / 2}\right) \psi \leq \frac{1}{2} \varepsilon \psi .
\end{aligned}
$$

Combining this with (5.54) yields (5.53).

It remains to show that $N$ is a supermartingale under $P^{\psi}$. As it is a local martingale, it suffices to show that it is bounded from below by a $P^{\psi}$-integrable random variable. To this end, first note from the definition of $w^{\psi}$ in (5.2) and Assumption 4.2 (e) and (f) that for every $(t, \mathbf{x}) \in \mathbf{D}$ and $y \geq-K_{\mathfrak{Y}}$,

$$
w^{\psi}(t, \mathbf{x}, y)=U(y)-U^{\prime}(y) \widetilde{w}(t, \mathbf{x}) \psi \geq U\left(-K_{\mathfrak{Y}}\right)-U^{\prime}\left(-K_{\mathfrak{Y}}\right) K_{\widetilde{w}} .
$$

By Assumption 4.2 (a) and the fact that $f \geq 0$, we obtain for each $u \in[0, T]$,

$$
I_{u}^{\psi}\left(\boldsymbol{v}, P^{\psi}\right) \geq U\left(-K_{\mathfrak{Y}}\right)-U^{\prime}\left(-K_{\mathfrak{Y}}\right) K_{\widetilde{w}}-w_{0}^{\psi}=: K_{I} .
$$

Using this and (5.56) yields for each $u \in[0, T]$,

$$
N_{u} \geq K_{I}-K_{U} \int_{0}^{T} K_{\text {up }}\left(t, \mathbf{X}_{t}\right) \mathrm{d} t .
$$


As $K_{\text {up }} \in L_{\mathfrak{P}}^{2}, N$ is bounded from below by a $P^{\psi}$-integrable random variable and therefore is a supermartingale. This completes the proof.

\subsection{Construction of a modified feedback control}

Proof of Lemma 4.9. For each $\psi>0$, define the functions $\widehat{\nu}, \check{\zeta}^{\psi *}, \check{\boldsymbol{\zeta}}^{\psi}: \mathbf{D}^{0} \rightarrow \mathbb{R}$ by

$$
\begin{aligned}
\widehat{\nu}(t, \mathbf{x}) & =-\frac{1}{2 \mathcal{C}_{\Sigma}}\left(\begin{array}{c}
\widetilde{\sigma} \\
\widetilde{\eta}
\end{array}\right)^{\top}\left(\begin{array}{cc}
S^{2} \mathcal{C}_{S S} & S \mathcal{C}_{S \Sigma} \\
S \mathcal{C}_{S \Sigma} & \mathcal{C}_{\Sigma \Sigma}
\end{array}\right)\left(\begin{array}{c}
\widetilde{\sigma} \\
\widetilde{\eta}
\end{array}\right), \\
\check{\boldsymbol{\zeta}}^{\psi *}(t, \mathbf{x}) & =\boldsymbol{\zeta}^{0}(\Sigma)+\widetilde{\boldsymbol{\zeta}} \psi+\widehat{\nu}_{1} \psi^{2}, \\
\check{\zeta}^{\psi}(t, \mathbf{x}) & =\boldsymbol{\zeta}^{0}(\Sigma)+\left(\widetilde{\boldsymbol{\zeta}} \psi+\widehat{\nu} \overrightarrow{\mathbf{e}}_{1} \psi^{2}\right) \mathbf{1}_{\{\underline{\Sigma}<\Sigma<\bar{\Sigma}\}}=\boldsymbol{\zeta}^{\psi}+\widehat{\nu} \overrightarrow{\mathbf{e}}_{1} \psi^{2} \mathbf{1}_{\{\underline{\Sigma}<\Sigma<\bar{\Sigma}\}},
\end{aligned}
$$

where the functions $\widetilde{\sigma}$ and $\widetilde{\eta}$ are the second and third component of $\widetilde{\zeta}$ defined in (4.5). That is, $\check{\boldsymbol{\zeta}}^{\psi}$ arises from $\zeta^{\psi}$ (cf. (4.6) ) by a perturbation of the first component (the drift of the implied volatility) by a term of order $O\left(\psi^{2}\right)$.

First, we show the asserted continuity of $\check{\zeta}^{\psi}$ and the extension property. It is easy to see from Assumption 4.2 (c) and (d) that the vega-gamma-vanna-volga vectors $\mathbf{c}$ and $\mathbf{v}$ are continuous on $\mathbf{D}^{0}$. Then also $\lambda$ (it is not hard to show that the two expressions on the right-hand side of (4.3) coincide whenever $\left.\mathcal{V}_{\Sigma \Sigma}-\frac{\mathbf{c}^{\top} \Psi \mathbf{v}}{\mathbf{c}^{\top} \Psi \mathbf{c}} \mathcal{C}_{\Sigma \Sigma}=0\right), \mu$, and hence $\widetilde{\zeta}$ are continuous on $\mathbf{D}^{0}$. Therefore, also $\widehat{\nu}$ and $\check{\zeta}^{\psi *}$ are continuous on $\mathbf{D}^{0}$, and it follows that $\check{\zeta}^{\psi}$ is continuous on $(0, T) \times \mathbf{G} \times(\underline{\Sigma}, \bar{\Sigma})$. By construction, $\check{\zeta}^{\psi *}$ is a continuous extension of $\left.\check{\zeta}^{\psi}\right|_{(0, T) \times \mathbf{G} \times(\underline{\Sigma}, \bar{\Sigma})}$ to $\mathbf{D}^{0}$.

Second, we show that the range of $\check{\zeta}^{\psi}$ is contained in $\mathbf{Z}=[\underline{\nu}, \bar{\nu}] \times[\underline{\sigma}, \bar{\sigma}] \times[\underline{\eta}, \bar{\eta}] \times[0, \bar{\xi}]$ for sufficiently small $\psi \in\left(0, \psi_{0}\right)$. To this end, it suffices to show that $\widetilde{\zeta}$ and $\widehat{\nu}$ are bounded on $\mathbf{D}=(0, T) \times \mathbf{G} \times[\underline{\Sigma}, \bar{\Sigma}]$. First, by (5.28) from the proof of Lemma 5.6 (a), we have

$$
|\widetilde{\boldsymbol{\zeta}}(t, \mathbf{x})| \leq \psi_{\max }|\mathbf{v}(t, \mathbf{x})|, \quad(t, \mathbf{x}) \in \mathbf{D}
$$

By Assumption 4.2 (d) $|\mathbf{v}|$ is bounded on $\mathbf{D}$, so that $\widetilde{\boldsymbol{\zeta}}$ is bounded on $\mathbf{D}$ as well. Second, using the boundedness of $\zeta$ as well as (4.17), it follows from (5.57) that $\widehat{\nu}$ is bounded on $\mathbf{D}$ as well. We conclude that there is $\psi_{0} \in(0,1)$ such that $\check{\zeta}^{\psi}(t, \mathbf{x}) \in \mathbf{Z}$ for each $(t, \mathbf{x}) \in \mathbf{D}$ and $\psi \in\left(0, \psi_{0}\right)$.

Third, we prove part (c). By (5.58), for each $(t, \mathbf{x}) \in \mathbf{D}$ and $\psi>0$, we have

$$
\left|\check{\boldsymbol{\zeta}}^{\psi}(t, \mathbf{x})-\boldsymbol{\zeta}^{\psi}(t, \mathbf{x})\right| \leq|\widehat{\nu}(t, \mathbf{x})| \psi^{2} .
$$

As we have argued above that $\widehat{\nu}$ is bounded on $\mathbf{D}$, there is $K_{0}>0$ such that (4.18) holds.

Fourth, we show part (b), i.e., that $b^{\mathcal{C}}\left(t, \mathbf{x} ; \check{\zeta}^{\psi}(t, \mathbf{x})\right)=0$. Fix $(t, \mathbf{x}) \in \mathbf{D}$. If $\Sigma \in\{\underline{\Sigma}, \bar{\Sigma}\}$, then $\check{\zeta}^{\psi}(t, \mathbf{x})=\zeta^{0}(\Sigma)$ by construction and the assertion is trivial. So suppose that $\Sigma \in(\underline{\Sigma}, \bar{\Sigma})$. By the representation (5.5) of $b^{\mathcal{C}}$, noting that the second and third components of $\check{\zeta}^{\psi}$ coincide with those of $\boldsymbol{\zeta}^{\psi}$, we have

$$
b^{\mathcal{C}}\left(\check{\boldsymbol{\zeta}}^{\psi}\right)=\mathbf{c}^{\top}\left(\check{\boldsymbol{\zeta}}^{\psi}-\zeta^{0}(\Sigma)\right)+\frac{1}{2}\left(\begin{array}{c}
\sigma^{\psi}-\Sigma \\
\eta^{\psi}
\end{array}\right)^{\top}\left(\begin{array}{cc}
S^{2} \mathcal{C}_{S S} & S \mathcal{C}_{S \Sigma} \\
S \mathcal{C}_{S \Sigma} & \mathcal{C}_{\Sigma \Sigma}
\end{array}\right)\left(\begin{array}{c}
\sigma^{\psi}-\Sigma \\
\eta^{\psi}
\end{array}\right) .
$$

We note that $\mathbf{c}^{\top}\left(\boldsymbol{\zeta}^{\psi}-\boldsymbol{\zeta}^{0}(\Sigma)\right)=\mathbf{c}^{\top} \widetilde{\boldsymbol{\zeta}} \psi=0$ by Lemma [5.6 (a), so that $\mathbf{c}^{\top}\left(\check{\boldsymbol{\zeta}}^{\psi}-\boldsymbol{\zeta}^{0}(\Sigma)\right)=$ $\mathbf{c}^{\top}\left(\check{\boldsymbol{\zeta}}^{\psi}-\boldsymbol{\zeta}^{\psi}\right)$. Using this together with the fact that $\sigma^{\psi}=\Sigma+\widetilde{\sigma} \psi$ and $\eta^{\psi}=\tilde{\eta} \psi$ (because $\Sigma \in(\underline{\Sigma}, \bar{\Sigma}))$ as well as the definition of $\widehat{\nu}$ yields

$$
b^{\mathcal{C}}\left(\check{\zeta}^{\psi}\right)=\mathbf{c}^{\top}\left(\check{\zeta}^{\psi}-\boldsymbol{\zeta}^{\psi}\right)-\mathcal{C}_{\Sigma} \widehat{\nu} \psi^{2}
$$

Now by (5.58) and the definition of $\mathbf{c}($ cf. (4.1) $), \mathbf{c}^{\top}\left(\check{\boldsymbol{\zeta}}^{\psi}-\boldsymbol{\zeta}^{\psi}\right)=\mathcal{C}_{\Sigma} \widehat{\nu} \psi^{2}$, so that $b^{\mathcal{C}}\left(\check{\boldsymbol{\zeta}}^{\psi}\right)=0$.

Finally, part (a) follows immediately from the construction of $\check{\zeta}^{\psi}$. 


\section{A Linearly constrained quadratic programming}

Lagrangian duality. We recall some basic Lagrange duality results from [8, Section 5.1.5]. Fix $n \in \mathbb{N}$ and functions $f, g, h: \mathbb{R}^{n} \rightarrow \mathbb{R}$. We refer to the problem

$$
\text { minimise } f(\mathbf{z}) \quad \text { subject to } \mathbf{z} \in \mathbb{R}^{n}, h(\mathbf{z})=0, g(\mathbf{z}) \leq 0,
$$

as the primal problem and denote by

$$
f^{*}=\inf \left\{f(\mathbf{z}): \mathbf{z} \in \mathbb{R}^{n}, h(\mathbf{z})=0, g(\mathbf{z}) \leq 0\right\}
$$

its optimal value. The corresponding Lagrangian is

$$
L(\mathbf{z}, \lambda, \mu)=f(\mathbf{z})+\lambda h(\mathbf{z})+\mu g(\mathbf{z}), \quad \mathbf{z} \in \mathbb{R}^{n}, \lambda, \mu \in \mathbb{R},
$$

and a pair $\left(\mu^{*}, \lambda^{*}\right)$ is called a Lagrange multiplier if

$$
f^{*}=\inf _{\mathbf{z} \in \mathbb{R}^{n}} L\left(\mathbf{z}, \lambda^{*}, \mu^{*}\right) \quad \text { and } \quad \mu^{*} \geq 0 .
$$

The dual problem for A.1 is

$$
\text { maximise } q(\lambda, \mu) \text { subject to } \lambda \in \mathbb{R}, \mu \geq 0 \text {, }
$$

where the dual function $q$ is

$$
q(\lambda, \mu)=\inf _{\mathbf{z} \in \mathbb{R}^{n}} L(\mathbf{z}, \lambda, \mu), \quad \lambda, \mu \in \mathbb{R} .
$$

Finally, $q^{*}=\sup \{q(\lambda, \mu): \lambda \in \mathbb{R}, \mu \geq 0\}$ denotes the optimal value of the dual problem.

A quadratic programming problem with linear equality and inequality constraints. The following lemma provides the solution to a primal problem with a strictly convex quadratic cost function and specific linear equality and inequality constraints.

Lemma A.1. Fix $n \in \mathbb{N}$, a diagonal matrix $D=\operatorname{diag}\left(d_{1}, \ldots, d_{n}\right) \in \mathbb{R}^{n \times n}$ with positive diagonal entries, and vectors $\mathbf{v}=\left(v_{1}, \ldots, v_{n}\right)^{\top}$ and $\mathbf{c}=\left(c_{1}, \ldots, c_{n}\right)^{\top}$ in $\mathbb{R}^{n}$ such that $c_{i} \neq 0$ for some $i \in\{1, \ldots, n-1\}$. Moreover, set $\mathbf{1}_{A}=1$ if $v_{n}-\frac{\mathbf{c}^{\top} D^{-1} \mathbf{v}}{\mathbf{c}^{\top} D^{-1} \mathbf{c}} c_{n}<0$ and $\mathbf{1}_{A}=0$ otherwise, and define

$$
\begin{aligned}
\lambda^{*} & =\frac{\mathbf{c}^{\top} D^{-1} \mathbf{v}-c_{n} v_{n} d_{n}^{-1} \mathbf{1}_{A}}{\mathbf{c}^{\top} D^{-1} \mathbf{c}-c_{n}^{2} d_{n}^{-1} \mathbf{1}_{A}}, \\
\mu^{*} & =\left(v_{n}-\lambda^{*} c_{n}\right)^{-} \\
\mathbf{z}^{*} & =D^{-1}\left(\mathbf{v}-\lambda^{*} \mathbf{c}+\mu^{*} \overrightarrow{\mathbf{e}}_{n}\right) .
\end{aligned}
$$

(a) $\mathbf{z}^{*}$ is the unique optimiser of the primal problem

$$
\text { minimise } \frac{1}{2} \mathbf{z}^{\top} \text { D } \mathbf{z}-\mathbf{v}^{\top} \mathbf{z} \quad \text { subject to } \mathbf{z} \in \mathbb{R}^{n}, \mathbf{c}^{\top} \mathbf{z}=0, z_{n} \geq 0,
$$

and satisfies the bound $\left|\mathbf{z}^{*}\right| \leq d_{\min }^{-1}|\mathbf{v}|$, where $d_{\min }=\min \left(d_{1}, \ldots, d_{n}\right)$.

(b) $\left(\lambda^{*}, \mu^{*}\right)$ is the unique optimiser of the dual problem for (A.5), which can be written as

$$
\text { maximise }-\frac{1}{2}\left(\mathbf{v}-\lambda \mathbf{c}+\mu \overrightarrow{\mathbf{e}}_{n}\right)^{\top} D^{-1}\left(\mathbf{v}-\lambda \mathbf{c}+\mu \overrightarrow{\mathbf{e}}_{n}\right) \quad \text { subject to } \lambda \in \mathbb{R}, \mu \geq 0 .
$$

The optimiser satisfies the bound

$$
\left|\lambda^{*} \mathbf{c}-\mu^{*} \overrightarrow{\mathbf{e}}_{n}\right| \leq\left(1+\frac{d_{\max }}{d_{\min }}\right)|\mathbf{v}|,
$$

where $d_{\max }=\max \left(d_{1}, \ldots, d_{n}\right)$. 
(c) The optimal values of the primal and dual problems coincide (i.e., there is no duality gap) and equal

$$
-\frac{1}{2} \mathbf{v}^{\top} \mathbf{z}^{*}
$$

(d) The triplet $\left(\mathbf{z}^{*}, \mu^{*}, \lambda^{*}\right)$ satisfies the optimality conditions

$$
\mathbf{z}^{*}=\arg \min _{\mathbf{z} \in \mathbb{R}^{n}} L\left(\mathbf{z}, \lambda^{*}, \mu^{*}\right), \quad \mathbf{c}^{\top} \mathbf{z}^{*}=0, \quad z_{n}^{*} \geq 0, \quad \mu^{*} \geq 0, \quad \mu^{*} z_{n}^{*}=0,
$$

where $L$ is the Lagrangian corresponding to the primal problem. Moreover, $\left(\lambda^{*}, \mu^{*}\right)$ is a Lagrange multiplier for the primal problem.

Proof. First of all, note that the Lagrangian

$$
L(\mathbf{z}, \lambda, \mu)=\frac{1}{2} \mathbf{z}^{\top} D \mathbf{z}-\mathbf{v}^{\top} \mathbf{z}+\lambda \mathbf{c}^{\top} \mathbf{z}-\mu \overrightarrow{\mathbf{e}}_{n}^{\top} \mathbf{z}
$$

corresponding to the primal problem is strictly convex over $\mathbf{z} \in \mathbb{R}^{n}$. Hence, the dual function $q(\lambda, \mu)=\inf _{\mathbf{z} \in \mathbb{R}^{n}} L(\mathbf{z}, \lambda, \mu)$ can be computed explicitly by substituting the solution $\mathbf{z}^{\prime}$ to the first-order condition $\mathbf{0}=\mathrm{D}_{\mathbf{z}} L\left(\mathbf{z}^{\prime}, \lambda, \mu\right)=D \mathbf{z}^{\prime}-\mathbf{v}+\lambda \mathbf{c}-\mu \overrightarrow{\mathbf{e}}_{n}$ back into $L\left(\mathbf{z}^{\prime}, \mu, \lambda\right)$. This yields

$$
q(\lambda, \mu)=-\frac{1}{2}\left(\mathbf{v}-\lambda \mathbf{c}+\mu \overrightarrow{\mathbf{e}}_{n}\right)^{\top} D^{-1}\left(\mathbf{v}-\lambda \mathbf{c}+\mu \overrightarrow{\mathbf{e}}_{n}\right)
$$

and thus the dual problem takes the form A.6.

The crucial part of the proof is to show that the triplet $\left(\mathbf{z}^{*}, \lambda^{*}, \mu^{*}\right)$ satisfies the optimality conditions (A.8). As $L$ is strictly convex over $\mathbf{z} \in \mathbb{R}^{n}$, the optimality conditions are equivalent to

$$
D \mathbf{z}^{*}-\mathbf{v}+\lambda^{*} \mathbf{c}-\mu^{*} \overrightarrow{\mathbf{e}}_{n}=0, \quad \mathbf{c}^{\top} \mathbf{z}^{*}=0, \quad z_{n}^{*} \geq 0, \quad \mu^{*} \geq 0, \quad \mu^{*} z_{n}^{*}=0 .
$$

Recall the definitions of $\lambda^{*}, \mu^{*}$, and $\mathbf{z}^{*}$ in A.2 A.4 and note that the assumption that $c_{i} \neq 0$ for some $i \in\{1, \ldots, n-1\}$ together with the positive definiteness of $D^{-1}$ ensures that $\lambda^{*}$ is well defined. The stationarity condition $D \mathbf{z}^{*}-\mathbf{v}+\lambda^{*} \mathbf{c}-\mu^{*} \overrightarrow{\mathbf{e}}_{n}=0$ holds by definition of $\mathbf{z}^{*}$. For the other conditions, we distinguish two cases. First, suppose that $\mathbf{1}_{A}=0$, i.e., $v_{n}-\frac{\mathbf{c}^{\top} D^{-1} \mathbf{v}}{\mathbf{c}^{\top} D^{-1} \mathbf{c}} c_{n} \geq 0$. Then $\lambda^{*}=\frac{\mathbf{c}^{\top} D^{-1} \mathbf{v}}{\mathbf{c}^{\top} D^{-1} \mathbf{c}}, \mu^{*}=0$, and $z_{n}^{*}=d_{n}^{-1}\left(v_{n}-\lambda^{*} c_{n}\right) \geq 0$. Moreover, $\mathbf{c}^{\top} \mathbf{z}^{*}=\mathbf{c}^{\top} D^{-1} \mathbf{v}-\lambda^{*} \mathbf{c}^{\top} D^{-1} \mathbf{c}=0$. Second, suppose that $\mathbf{1}_{A}=1$, i.e., $v_{n}-\frac{\mathbf{c}^{\top} D^{-1} \mathbf{v}}{\mathbf{c}^{\top} D^{-1} \mathbf{c}} c_{n}<0$, or, equivalently (multiply by $\mathbf{c}^{\top} D^{-1} \mathbf{c}$, add and subtract $c_{n}^{2} v_{n} d_{n}^{-1}$, and then divide by $\left.\mathbf{c}^{\top} D^{-1} \mathbf{c}-c_{n}^{2} d_{n}^{-1}>0\right), v_{n}-\lambda^{*} c_{n}<0$. Then $\mu^{*}>0$ and $z_{n}^{*}=0$ by definition of $\mu^{*}$ and $\mathbf{z}^{*}$. Finally, setting $c=\mathbf{c}^{\top} D^{-1} \mathbf{c}$ and $v=\mathbf{c}^{\top} D^{-1} \mathbf{v}$ for brevity,

$$
\begin{aligned}
\mathbf{c}^{\top} \mathbf{z}^{*} & =\mathbf{c}^{\top} D^{-1}\left(\mathbf{v}-\lambda^{*} \mathbf{c}+\mu^{*} \overrightarrow{\mathbf{e}}_{n}\right)=v-\lambda^{*} c+\mu^{*} c_{n} d_{n}^{-1}=v-\lambda^{*} c-\left(v_{n}-\lambda^{*} c_{n}\right) c_{n} d_{n}^{-1} \\
& =v-\lambda^{*}\left(c-c_{n}^{2} d_{n}^{-1}\right)-c_{n} v_{n} d_{n}^{-1}=v-\left(v-c_{n} v_{n} d_{n}^{-1}\right)-c_{n} v_{n} d_{n}^{-1}=0 .
\end{aligned}
$$

So, A.9 holds in both cases. By the characterisation of primal optimal solutions [8, Proposition 5.1.5], this implies that $\mathbf{z}^{*}$ is an optimiser for the primal problem, that $\left(\lambda^{*}, \mu^{*}\right)$ is a Lagrange multiplier, and that there is no duality gap. Moreover, $\left(\lambda^{*}, \mu^{*}\right)$ is an optimiser for the dual problem by a corollary [8, Proposition 5.1.4 (a)] of the weak duality theorem [8, Proposition 5.1.3]. As the primal and dual problems are strictly convex and strictly concave, respectively, the optimisers are unique.

Plugging the optimiser $\left(\lambda^{*}, \mu^{*}\right)$ of the dual problem into the cost function of the dual problem (A.6) and using the definition of $\mathbf{z}^{*}$, the optimal value $q^{*}$ (of both the primal and the dual problem) reads

$$
q^{*}:=-\frac{1}{2}\left(\mathbf{v}-\lambda^{*} \mathbf{c}+\mu^{*} \overrightarrow{\mathbf{e}}_{n}\right)^{\top} D^{-1}\left(\mathbf{v}-\lambda^{*} \mathbf{c}+\mu \overrightarrow{\mathbf{e}}_{n}\right)=-\frac{1}{2}\left(\mathbf{v}-\lambda^{*} \mathbf{c}+\mu^{*} \overrightarrow{\mathbf{e}}_{n}\right)^{\top} \mathbf{z}^{*} .
$$

Now, note that $\mathbf{c}^{\top} \mathbf{z}^{*}=0$ and $\mu^{*} \overrightarrow{\mathbf{e}}_{n}^{\top} \mathbf{z}^{*}=\mu^{*} z_{n}^{*}=0$ by (A.9). Hence, $q^{*}=-\frac{1}{2} \mathbf{v}^{\top} \mathbf{z}^{*}$. 
Next, using that $\mathbf{z}^{*}$ achieves the optimal value $-\frac{1}{2} \mathbf{v}^{\top} \mathbf{z}^{*}$ for the primal problem and applying the Cauchy-Schwarz inequality, we obtain

$$
\frac{1}{2} d_{\min }\left|\mathbf{z}^{*}\right|^{2} \leq \frac{1}{2}\left(\mathbf{z}^{*}\right)^{\top} D \mathbf{z}^{*}=\frac{1}{2} \mathbf{v}^{\top} \mathbf{z}^{*} \leq \frac{1}{2}|\mathbf{v}|\left|\mathbf{z}^{*}\right| .
$$

This yields the last claim of part (a). Finally, using (A.4), the triangle inequality, and the bound $\left|\mathbf{z}^{*}\right| \leq d_{\min }^{-1}|\mathbf{v}|$ which we just proved, we obtain

$$
\left|\lambda^{*} \mathbf{c}-\mu^{*} \overrightarrow{\mathbf{e}}_{n}\right|=\left|D \mathbf{z}^{*}-\mathbf{v}\right| \leq\left|D \mathbf{z}^{*}\right|+|\mathbf{v}| \leq d_{\max }\left|\mathbf{z}^{*}\right|+|\mathbf{v}| \leq\left(1+\frac{d_{\max }}{d_{\min }}\right)|\mathbf{v}| .
$$

This proves the last claim of part (b) and thereby concludes the proof.

\section{References}

[1] B. Acciaio, M. Beiglböck, F. Penkner, and W. Schachermayer, A model-free version of the fundamental theorem of asset pricing and the super-replication theorem, Math. Finance 26 (2016), no. 2, 233-251.

[2] H. Ahn, A. Muni, and G. Swindle, Misspecified asset price models and robust hedging strategies, Appl. Math. Finance 4 (1997), no. 1, 21-36.

[3] _ Optimal hedging strategies for misspecified asset price models, Appl. Math. Finance 6 (1999), no. 3, 197-208.

[4] M. Avellaneda and R. Buff, Combinatorial implications of nonlinear uncertain volatility models: the case of barrier options, Appl. Math. Finance 6 (1999), no. 1, 1-18.

[5] M. Avellaneda, A. Levy, and A. Parás, Pricing and hedging derivative securities in markets with uncertain volatilities, Appl. Math. Finance 2 (1995), no. 2, 73-88.

[6] M. Avellaneda and A. Parás, Managing the volatility risk of portfolios of derivative securities: the Lagrangian uncertain volatility model, Appl. Math. Finance 3 (1996), no. 1, 21-52.

[7] M. Beiglböck, P. Henry-Labordère, and F. Penkner, Model-independent bounds for option prices-a mass transport approach, Finance Stoch. 17 (2013), no. 3, 477-501.

[8] D. Bertsekas, Nonlinear Programming, 2nd ed., Athena Scientific, Belmont, 1999.

[9] S. Biagini, B. Bouchard, C. Kardaras, and M. Nutz, Robust fundamental theorem for continuous processes, Math. Finance (2017+), to appear.

[10] B. Bouchard and M. Nutz, Arbitrage and duality in nondominated discrete-time models, Ann. Appl. Probab. 25 (2015), no. 2, 823-859.

[11] A. Brace, B. Goldys, F. Klebaner, and R. Womersley, Market model of stochastic implied volatility with application to the BGM model, Preprint, 2001.

[12] H. Brown, D. Hobson, and L. C. G. Rogers, Robust hedging of barrier options, Math. Finance 11 (2001), no. 3, 285-314.

[13] R. Carmona and S. Nadtochiy, Local volatility dynamic models, Finance Stoch. 13 (2009), no. 1, 1-48.

[14] _ Tangent models as a mathematical framework for dynamic calibration, Int. J. Theor. Appl. Finance 14 (2011), no. 1, 107-135.

[15] — Tangent Lévy market models, Finance Stoch. 16 (2012), no. 1, 63-104.

[16] P. Carr and R. Lee, Hedging variance options on continuous semimartingales, Finance Stoch. 14 (2010), no. 2, 179-207.

[17] A. Cox and J. Obłój, Robust hedging of double touch barrier options, SIAM J. Financ. Math. 2 (2011), no. $1,141-182$.

[18] , Robust pricing and hedging of double no-touch options, Finance Stoch. 15 (2011), no. 3, 573-605.

[19] M. Davis, Complete-market models of stochastic volatility, Proc. R. Soc. Lond. A 460 (2004), no. 2041, 11-26. 
[20] M. Davis and J. Obłój, Market completion using options, Advances in Mathematics of Finance (L. Stettner, ed.), Polish Academy of Sciences, Institute of Mathematics, Warsaw, 2008, pp. 4960 .

[21] L. Denis and C. Martini, A theoretical framework for the pricing of contingent claims in the presence of model uncertainty, Ann. Appl. Probab. 16 (2006), no. 2, 827-852.

[22] Y. Dolinsky and H. M. Soner, Martingale optimal transport and robust hedging in continuous time, Probab. Theory Relat. Fields 160 (2014), no. 1-2, 391-427.

[23] J.-P. Fouque and B. Ren, Approximation for option prices under uncertain volatility, SIAM J. Financ. Math. 5 (2014), no. 1, 260-383.

[24] R. Frey, Superreplication in stochastic volatility models and optimal stopping, Finance Stoch. 4 (2000), no. 2, 161-187.

[25] A. Galichon, P. Henry-Labordère, and N. Touzi, A stochastic control approach to no-arbitrage bounds given marginals, with an application to lookback options, Ann. Appl. Probab. 24 (2014), no. 1, 312336.

[26] J. Goodman and D. Ostrov, An option to reduce transaction costs, SIAM J. Financ. Math. 2 (2011), no. $1,512-537$.

[27] L. Hansen and T. Sargent, Robust control and model uncertainty, Am. Econ. Rev. 91 (2001), no. 2, 60-66.

[28] S. Herrmann, J. Muhle-Karbe, and F. Seifried, Hedging with small uncertainty aversion, Finance Stoch. 21 (2017), no. 1, 1-64.

[29] D. Hobson, Robust hedging of the lookback option, Finance Stoch. 2 (1998), no. 4, 329-347.

[30] D. Hobson and M. Klimmek, Model-independent hedging strategies for variance swaps, Finance Stoch. 16 (2012), no. 4, 611-649.

[31] _ Robust price bounds for the forward starting straddle, Finance Stoch. 19 (2015), no. 1, 189214.

[32] D. Hobson and A. Neuberger, Robust bounds for forward start options, Math. Finance 22 (2012), no. $1,31-56$.

[33] F. Hubalek, J. Kallsen, and L. Krawczyk, Variance-optimal hedging for processes with stationary independent increments, Ann. Appl. Prob. 16 (2006), no. 2, 853-885.

[34] J. Hull and A. White, Hedging the risks from writing foreign currency options, J. Int. Money Finance 6 (1987), no. 2, 131-152.

[35] J. Jacod and P. Protter, Risk-neutral compatibility with option prices, Finance Stoch. 14 (2010), no. 2, $285-315$.

[36] J. Kallsen and P. Krühner, On a Heath-Jarrow-Morton approach for stock options, Finance Stoch. 19 (2015), no. 3, 583-615.

[37] J. Kallsen and J. Muhle-Karbe, Option pricing and hedging with small transaction costs, Math. Finance 25 (2015), no. 4, 702-723.

[38] I. Karatzas and S. Shreve, Brownian Motion and Stochastic Calculus, 2nd ed., Springer, Berlin, 1998.

[39] O. Ledoit, P. Santa-Clara, and S. Yan, Relative pricing of options with stochastic volatility, Preprint, 2002.

[40] T. Lyons, Uncertain volatility and the risk-free synthesis of derivatives, Appl. Math. Finance 2 (1995), no. $2,117-133$.

[41] _ Derivatives as tradable assets, Seminario de Matemática Financiera MEFF-UAM, vol. 2, 1997, pp. 213-232.

[42] F. Maccheroni, M. Marinacci, and A. Rustichini, Ambiguity aversion, robustness, and the variational representation of preferences, Econometrica 74 (2006), no. 6, 1447-1498.

[43] P. Maenhout, Robust portfolio rules and asset pricing, Rev. Financ. Stud. 17 (2004), no. 4, 951-983.

[44] M. Musiela and M. Rutkowski, Martingale Methods in Financial Modelling, 2nd ed., Springer, Berlin, 2005.

[45] A. Neuberger, The log contract, J. Portfolio Manag. 20 (1994), no. 2, 74-80. 
[46] A. Neufeld and M. Nutz, Superreplication under volatility uncertainty for measurable claims, Electron. J. Probab. 18 (2013), no. 48, 1-14.

[47] M. Nutz, Superreplication under model uncertainty in discrete time, Finance Stoch. 18 (2014), no. 4, 791-803.

[48] J. Obłój and F. Ulmer, Performance of robust hedges for digital double barrier options, Int. J. Theor. Appl. Finance 15 (2012), no. 1, 1250003.

[49] D. Possamaï, G. Royer, and N. Touzi, On the robust superhedging of measurable claims, Electron. Commun. Probab. 18 (2013), no. 95, 1-13.

[50] R. Rebonato, Volatility and Correlation, 2nd ed., John Wiley \& Sons, Hoboken, 2004.

[51] L. C. G. Rogers and D. Williams, Diffusions, Markov Processes, and Martingales, 2nd ed., vol. 2, Cambridge University Press, Cambridge, 2000.

[52] M. Romano and N. Touzi, Contingent claims and market completeness in a stochastic volatility model, Math. Finance 7 (1997), no. 4, 399-412.

[53] P. Schönbucher, A market model for stochastic implied volatility, Phil. Trans. R. Soc. Lond. A 357 (1999), no. 1758, 2071-2092.

[54] M. Schweizer and J. Wissel, Arbitrage-free market models for option prices: the multi-strike case, Finance Stoch. 12 (2008), no. 4, 469-505.

[55] — Term structures of implied volatilities: absence of arbitrage and existence results, Math. Finance 18 (2008), no. 1, 77-104.

[56] L. Scott, Random variance option pricing: empirical tests of the model and delta-sigma hedging, Adv. Futures Options Res. 5 (1991), 113-135.

[57] A. Whalley and P. Wilmott, An asymptotic analysis of an optimal hedging model for option pricing with transaction costs, Math. Finance 7 (1997), no. 3, 307-324.

[58] P. Wilmott, Paul Wilmott on Quantitative Finance, 2nd ed., John Wiley \& Sons, Hoboken, 2006. 